\title{
Asymmetric Synthesis of Fluorinated Cyclic $\beta$-Amino Acid Derivatives Through Cross Metathesis
}

Santos Fustero,,$^{,+, \neq}$María Sánchez-Roselló, ${ }^{\dagger}$, Juan F. SanzCervera, ${ }^{\dagger, \ddagger}$ José Luis Aceña, ${ }^{\ddagger}$ Carlos del Pozo, ${ }^{\dagger}$ Begoña Fernández, ${ }^{\dagger}$ Ana Bartolomé, ${ }^{\dagger}$ and Amparo Asensio ${ }^{\dagger}$

${ }^{\dagger}$ Departamento de Química Orgánica. Universidad de Valencia. E-46100 Burjassot, Spain

${ }^{\ddagger}$ Laboratorio de Moléculas Orgánicas, Centro de Investigación Príncipe Felipe, E-46013 Valencia, Spain.

Email Address: santos.fustero@uv.es

\section{SUPPORTING INFORMATION}

\section{Table of contents:}

General methods

General procedure A

General procedure B

General procedure $\mathrm{C}$

General procedure D

General procedure E

General procedure F

Synthesis of $\mathbf{1 2 b}$

Synthesis of 12c 
Synthesis of $\mathbf{1 b} \quad$ S8

$\begin{array}{ll}\text { Synthesis of 1c } & \text { S8 }\end{array}$

$\begin{array}{ll}\text { Synthesis of } \mathbf{5 a} & \text { S9 }\end{array}$

$\begin{array}{ll}\text { Synthesis of } \mathbf{5 b} & \mathrm{S} 10\end{array}$

$\begin{array}{ll}\text { Synthesis of 5c } & \text { S10 }\end{array}$

$\begin{array}{ll}\text { Synthesis of } \mathbf{6 a} & \mathrm{S} 11\end{array}$

$\begin{array}{ll}\text { Synthesis of } \mathbf{6 b} & \mathrm{S} 12\end{array}$

$\begin{array}{ll}\text { Synthesis of 6c } & \mathrm{S} 12\end{array}$

$\begin{array}{ll}\text { Synthesis of 7a } & \text { S13 }\end{array}$

$\begin{array}{ll}\text { Synthesis of } \mathbf{7 b} & \text { S14 }\end{array}$

$\begin{array}{ll}\text { Synthesis of 7c } & \text { S14 }\end{array}$

$\begin{array}{ll}\text { Synthesis of } \mathbf{8 a} & \mathrm{S} 15\end{array}$

$\begin{array}{ll}\text { Synthesis of } \mathbf{8 b} & \text { S16 }\end{array}$

$\begin{array}{ll}\text { Synthesis of 8c } & \text { S17 }\end{array}$

$\begin{array}{ll}\text { Synthesis of }(+)-5 d & \text { S17 }\end{array}$

$\begin{array}{ll}\text { Synthesis of (+)-6d } & \text { S18 }\end{array}$

$\begin{array}{lr}\text { Synthesis of }(+)-7 d & \text { S19 }\end{array}$

$\begin{array}{lr}\text { Synthesis of }(+)-8 d & \text { S19 }\end{array}$

$\begin{array}{ll}\text { Synthesis of }(+)-13 & \text { S21 }\end{array}$

$\begin{array}{ll}\text { Synthesis of }(+)-14 & \text { S22 }\end{array}$

$\begin{array}{ll}\text { Synthesis of }(+)-15 & \text { S23 }\end{array}$

$\begin{array}{ll}\text { X-ray data of }(+)-15 & \text { S24 }\end{array}$

$\begin{array}{ll}\text { NMR spectra of 12b } & \text { S27-S29 }\end{array}$

$\begin{array}{ll}\text { NMR spectra of 12c } & \text { S30-S32 }\end{array}$ 
NMR spectra of $\mathbf{1 b}$

NMR spectra of 1c

S36-S38

NMR spectra of $\mathbf{5 a}$

S39-S41

NMR spectra of $5 \mathbf{b}$

S42-S44

NMR spectra of 5c

S45-S47

NMR spectra of $\mathbf{6 a}$

S48-S50

NMR spectra of $\mathbf{6 b}$

S51-S53

NMR spectra of $\mathbf{6 c}$

S54-S56

NMR spectra of 7a

S57-S59

NMR spectra of $\mathbf{7 b}$

S60-S62

NMR spectra of 7c

S63-S67

NMR spectra of $\mathbf{8 a}$

S66-S70

NMR spectra of $\mathbf{8 b}$

S71-S73

NMR spectra of 8c

S74-S76

NMR spectra of $\mathbf{5 d}$

S77-S79

NMR spectra of $\mathbf{6 d}$

S80-S82

NMR spectra of 7d

S83-S85

NMR spectra of $\mathbf{8 d}$

S86-S88

NMR spectra of the $(2 R, 3 R)$ diastereoisomer of $\mathbf{8 d}$

S89-S91

NMR spectra of $\mathbf{1 3}$

S92-S94

NMR spectra of 14

S95-S97

NMR spectra of $\mathbf{1 5}$

S98-S100 


\section{General methods.}

All reactions were carried out under argon or nitrogen atmosphere. The solvents were purified prior to use: THF, $\mathrm{Et}_{2} \mathrm{O}$ and $\mathrm{PhMe}$ were distilled from sodium/benzophenone; $\mathrm{CH}_{2} \mathrm{Cl}_{2}$ and $\mathrm{CCl}_{4}$ were distilled from calcium hydride. Anhydrous EtOAc was purchased from Aldrich. All reagents were used as received. The reactions were monitored with the aid of thin-layer chromatography (TLC) on $0.25 \mathrm{~mm}$ E. Merck precoated silica gel plates. Visualization was carried out with UV light and aqueous ceric ammonium molybdate solution or potassium permanganate stain. Flash column chromatography was performed with the indicated solvents on silica gel 60 (particle size 0.040-0.063 mm). Melting points were measured on a Büchi B-540 apparatus and are uncorrected. Optical rotations were measured on a Jasco P-1020 polarimeter. Microwave reactions were conducted on a Biotage Initiator apparatus. ${ }^{1} \mathrm{H},{ }^{13} \mathrm{C}$, and ${ }^{19} \mathrm{~F}$ NMR spectra were recorded on a $300 \mathrm{MHz}$ Bruker AC300 spectrometer. Chemical shifts are given in

ppm $(\delta)$, referenced to the residual proton resonances of the solvents or fluorotrichloromethane in ${ }^{19} \mathrm{~F}$ NMR experiments. Coupling constants $(J)$ are given in Hertz $(\mathrm{Hz})$. The letters $\mathrm{m}, \mathrm{s}, \mathrm{d}, \mathrm{t}$, and $\mathrm{q}$ stand for multiplet, singlet, doublet, triplet, and quartet, respectively. The letters br indicate that the signal is broad. High-resolution mass spectra were carried out by the Universidad de Valencia Mass Spectrometry Service.

\section{General Procedure for the synthesis of difluorinated carboxylic acids (General Procedure}

\section{A).}

Bis(2-methoxyethyl)aminosulfur trifluoride (Deoxofluor $\left.{ }^{\circledR}\right)$ (1.7 equiv) and EtOH (0.2 equiv) were added to a cold $\left(0{ }^{\circ} \mathrm{C}\right)$ solution of the $\alpha$-ketoester $\left(1.0\right.$ equiv) in $\mathrm{CH}_{2} \mathrm{Cl}_{2}(0.3 \mathrm{M})$. The reaction mixture was stirred overnight and then quenched with sat. aq. $\mathrm{NaHCO}_{3}$ and extracted 
with $\mathrm{CH}_{2} \mathrm{Cl}_{2}$. The combined organic layers were washed with $1 \mathrm{M} \mathrm{HCl}$ and brine, dried over $\mathrm{Na}_{2} \mathrm{SO}_{4}$, filtered, and the volatiles were removed under reduced pressure to afford the intermediate difluorinated esters. These were dissolved in a 3.5:1 $\mathrm{THF} / \mathrm{H}_{2} \mathrm{O}$ mixture $(0.3 \mathrm{M})$, cooled to $0{ }^{\circ} \mathrm{C}$, and then $\mathrm{LiOH} \cdot \mathrm{H}_{2} \mathrm{O}$ (3 equiv) was added. After stirring for $5 \mathrm{~h}$, the reaction mixture was acidified with $1 \mathrm{M} \mathrm{HCl}$ and extracted with EtOAc. The organic layers were washed with brine, dried over $\mathrm{Na}_{2} \mathrm{SO}_{4}$, and filtered. The filtrate was concentrated and purified by distilling it under reduced pressure.

\section{General Procedure for the synthesis of fluorinated imidoyl chlorides (General Procedure} B).

$\mathrm{PPh}_{3}$ (3.0 equiv) and $\mathrm{Et}_{3} \mathrm{~N}$ (1.2 equiv) were added to a cold $\left(0^{\circ} \mathrm{C}\right)$ solution of the carboxylic acid (1.0 equiv) in $\mathrm{CCl}_{4}(1 \mathrm{M})$. After stirring for $10 \mathrm{~min}$, $p$-anisidine (1.2 equiv) was added and the mixture was stirred at reflux for $3 \mathrm{~h}$. The solvents were then removed under reduced pressure, and the residue was suspended in hexane and filtered. The filtrate was concentrated and purified by distilling it under reduced pressure.

\section{General Procedure for the cross-metathesis reaction (General Procedure C).}

Ethyl acrylate (5.0 equiv) and second generation ruthenium alkylidene catalyst 9 ( 0.05 equiv) were added successively to a solution of the fluorinated imidoyl chloride (1.0 equiv) in PhMe $(0.25 \mathrm{M})$. The mixture was stirred at $95{ }^{\circ} \mathrm{C}$ under argon overnight. The solvent was then removed under reduced pressure and the crude reaction mixture was purified by means of flash column chromatography on silica gel with the appropriate solvents. 
General Procedure for the chemoselective hydrogenation of olefins (General Procedure D). Palladium on active carbon ( $10 \% \mathrm{wt}, 0.2$ equiv) was added to a solution of the enoate ( 1.0 equiv) in EtOAc $(0.05 \mathrm{M})$. The resulting suspension was stirred in a medium pressure reactor with hydrogen $(4 \mathrm{~atm})$ for $24 \mathrm{~h}$. The reaction mixture was then filtered through Celite ${ }^{\circledR}$. The filtrate was concentrated and purified by means of flash chromatography on silica gel with the appropriate solvents.

\section{General Procedure for the Dieckmann-type cyclization (General Procedure E).}

$N$-Buthyllithium (2.5 $\mathrm{M}$ in hexane, 2.1 equiv) was added dropwise to a cold $\left(-30{ }^{\circ} \mathrm{C}\right)$ solution of diisopropylamine (2.1 equiv) in THF ( $0.25 \mathrm{M})$. After stirring for $30 \mathrm{~min}$, the mixture was cooled to $-78{ }^{\circ} \mathrm{C}$ and a solution of the imidoyl chloride (1.0 equiv) in THF ( $\left.0.12 \mathrm{M}\right)$ was added slowly. After stirring for $1 \mathrm{~h}$ at $-78{ }^{\circ} \mathrm{C}$, the reaction was quenched with sat. aq. $\mathrm{NH}_{4} \mathrm{Cl}$ and the aqueous layer was extracted with $\mathrm{CH}_{2} \mathrm{Cl}_{2}$. The combined organic layers were washed with brine and dried over $\mathrm{Na}_{2} \mathrm{SO}_{4}$. The filtrate was concentrated and purified by means of flash chromatography on silica gel with the appropriate solvents.

\section{General Procedure for the hydrogenation of enamino esters (General Procedure F).}

Palladium on active carbon (10\% wt, 0.2 equiv) and $\mathrm{NH}_{4} \mathrm{CO}_{2} \mathrm{H}$ (10.0 equiv) were added to a solution of the enamino ester $(1.0$ equiv) in $\mathrm{EtOH}(0.1 \mathrm{M})$. The mixture was stirred in a sealed tube in a microwave reactor at $100{ }^{\circ} \mathrm{C}$ for $45 \mathrm{~min}$. The reaction was then filtered. The filtrate was concentrated and purified by means of flash chromatography on silica gel with the appropriate solvents. 
Synthesis of 2,2-difluoro-5-hexenoic acid, $12 b$.

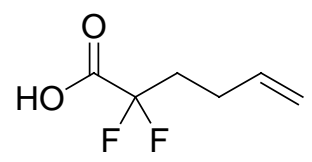

By means of General Procedure A, from $937 \mathrm{mg}(6.00 \mathrm{mmol})$ of ethyl 2-oxo-5-hexenoate 11b, ${ }^{1}$ $766 \mathrm{mg}$ of $\mathbf{1 2 b}$ were obtained as a colorless oil ( $85 \%$ yield). Bp: $30-33{ }^{\circ} \mathrm{C}\left(10^{-2}\right.$ Torr $) .{ }^{1} \mathrm{H}$ NMR $\left(300 \mathrm{MHz}, \mathrm{CDCl}_{3}\right) \delta 2.08-2.24(\mathrm{~m}, 4 \mathrm{H}), 4.98(\mathrm{~d}, J=11.1 \mathrm{~Hz}, 1 \mathrm{H}), 5.03(\mathrm{~d}, J=17.9 \mathrm{~Hz}, 1 \mathrm{H})$, 5.69-5.78 (m, 1H), $10.22(\mathrm{br}, 1 \mathrm{H}) .{ }^{13} \mathrm{C} \mathrm{NMR}\left(75.5 \mathrm{MHz}, \mathrm{CDCl}_{3}\right) \delta 25.5\left(\mathrm{t},{ }^{3} J_{\mathrm{CF}}=4.6 \mathrm{~Hz}\right), 33.5(\mathrm{t}$, $\left.{ }^{2} J_{\mathrm{CF}}=23.0 \mathrm{~Hz}\right), 115.7\left(\mathrm{t},{ }^{1} J_{\mathrm{CF}}=250.4 \mathrm{~Hz}\right), 116.2,135.5,168.7\left(\mathrm{t},{ }^{2} J_{\mathrm{CF}}=33.6 \mathrm{~Hz}\right) .{ }^{19} \mathrm{~F} \mathrm{NMR}$ $\left(282.4 \mathrm{MHz}, \mathrm{CDCl}_{3}\right) \delta-107.29\left(\mathrm{t}, J_{\mathrm{FH}}=16.0 \mathrm{~Hz}, 2 \mathrm{~F}\right)$. HRMS (EI) calcd for $\mathrm{C}_{6} \mathrm{H}_{8} \mathrm{~F}_{2} \mathrm{O}_{2}\left(\mathrm{M}^{+}\right)$: 150.0942, found: 150.0452 .

Synthesis of 2,2-difluoro-6-heptenoic acid, 12c.

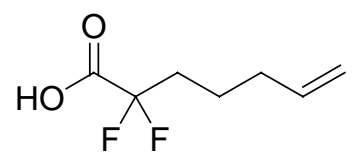

By means of General Procedure A, from 760 mg (4.47 mmol) of ethyl 2-oxo-6-heptenoate 11c, ${ }^{1}$ $623 \mathrm{mg}$ of $12 \mathrm{c}$ were obtained as a colorless oil ( $85 \%$ yield). Bp: $38-41{ }^{\circ} \mathrm{C}\left(10^{-2}\right.$ Torr $) .{ }^{1} \mathrm{H}$ NMR $\left(300 \mathrm{MHz}, \mathrm{CDCl}_{3}\right) \delta 1.51-1.61(\mathrm{~m}, 2 \mathrm{H}), 2.01-2.11(\mathrm{~m}, 4 \mathrm{H}), 4.94-5.02(\mathrm{~m}, 2 \mathrm{H})$, 5.64-5.77 (m, 1H), $9.57(\mathrm{br}, 1 \mathrm{H}) .{ }^{13} \mathrm{C} \mathrm{NMR}\left(75.5 \mathrm{MHz}, \mathrm{CDCl}_{3}\right) \delta 20.5\left(\mathrm{t},{ }^{3} J_{\mathrm{CF}}=4.0 \mathrm{~Hz}\right), 32.8,33.6\left(\mathrm{t},{ }^{2} J_{\mathrm{CF}}=\right.$ 23.0 Hz), 115.9, 137.1, 168.9. ${ }^{19} \mathrm{~F}$ NMR $\left(282.4 \mathrm{MHz}, \mathrm{CDCl}_{3}\right) \delta-107.13\left(\mathrm{t}, J_{\mathrm{FH}}=17.0 \mathrm{~Hz}, 2 \mathrm{~F}\right)$. HRMS (EI) calcd for $\mathrm{C}_{7} \mathrm{H}_{10} \mathrm{~F}_{2} \mathrm{O}_{2}\left(\mathrm{M}^{+}\right)$: 164.0649, found: 164.0691 .

\footnotetext{
${ }^{1}$ Macritchie, J. A.; Silcock, A.; Willis, C. L. Tetrahedron Asymmetry, 1997, 8, 3895-3902.
} 


\section{Synthesis of 1-chloro-2,2-difluoro- $N$-(4-methoxyphenyl)-5-hexen-1-imine, 1 b.}

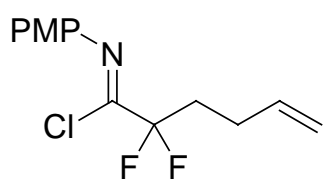

By means of General Procedure B, from $500 \mathrm{mg}(3.33 \mathrm{mmol})$ of 12b, $638 \mathrm{mg}$ of $\mathbf{1 b}$ were obtained as a colorless oil (70\% yield). Bp: $70-72{ }^{\circ} \mathrm{C}\left(2 \cdot 10^{-2}\right.$ Torr $) .{ }^{1} \mathrm{H}$ NMR $\left(300 \mathrm{MHz}, \mathrm{CDCl}_{3}\right)$ $\delta 2.37-2.43(\mathrm{~m}, 4 \mathrm{H}), 3.86(\mathrm{~s}, 3 \mathrm{H}), 5.07(\mathrm{dd}, J=10.2,1.5 \mathrm{~Hz}, 1 \mathrm{H}), 5.14(\mathrm{dd}, J=17.1,1.5 \mathrm{~Hz}, 1 \mathrm{H})$, 5.84-5.94 (m, 1H), $6.96(\mathrm{~d}, J=9.0 \mathrm{~Hz}, 2 \mathrm{H}), 7.17(\mathrm{~d}, J=9.0 \mathrm{~Hz}, 2 \mathrm{H}) .{ }^{13} \mathrm{C} \mathrm{NMR}(75.5 \mathrm{MHz}$, $\left.\mathrm{CDCl}_{3}\right) \delta 26.2\left(\mathrm{t},{ }^{3} J_{\mathrm{CF}}=4.3 \mathrm{~Hz}\right), 34.2\left(\mathrm{t},{ }^{2} J_{\mathrm{CF}}=23.6 \mathrm{~Hz}\right), 55.4,114.1,115.7,118.0\left(\mathrm{t},{ }^{1} J_{\mathrm{CF}}=246.7\right.$ $\mathrm{Hz}), 123.1,136.3,137.0,137.3\left(\mathrm{t},{ }^{2} J_{\mathrm{CF}}=36.8 \mathrm{~Hz}\right), 158.5 .{ }^{19} \mathrm{~F}$ NMR $\left(282.4 \mathrm{MHz}, \mathrm{CDCl}_{3}\right) \delta-$ $99.24\left(\mathrm{t}, J_{\mathrm{FH}}=15.5 \mathrm{~Hz}, 2 \mathrm{~F}\right)$. HRMS $(\mathrm{EI})$ calcd for $\mathrm{C}_{13} \mathrm{H}_{14} \mathrm{ClF}_{2} \mathrm{NO}\left(\mathrm{M}^{+}\right)$: 273.0732, found: 273.0650. Anal. calcd for $\mathrm{C}_{13} \mathrm{H}_{14} \mathrm{ClF}_{2} \mathrm{NO}$ : C, 57.05; H, 5.16; N, 5.12, found: C, 56.89; H, 5.03; $\mathrm{N}, 4.98$.

\section{Synthesis of 1-chloro-2,2-difluoro- $N$-(4-methoxyphenyl)-6-hepten-1-imine, $1 \mathrm{c}$.}

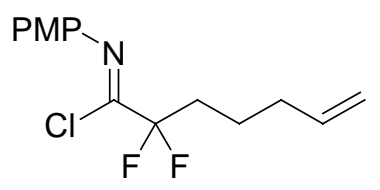

By means of General Procedure B, from $465 \mathrm{mg}(2.83 \mathrm{mmol})$ of 12c, $513 \mathrm{mg}$ of 1c were obtained as a colorless oil (63\% yield). Bp: $75-77^{\circ} \mathrm{C}\left(2 \cdot 10^{-2}\right.$ Torr $) .{ }^{1} \mathrm{H}$ NMR $\left(300 \mathrm{MHz}, \mathrm{CDCl}_{3}\right)$ $\delta$ 1.59-1.69 (m, 2H), 2.06-2.23 (m, 4H), $3.75(\mathrm{~s}, 3 \mathrm{H}), 4.94(\mathrm{dd}, J=10.3,1.5 \mathrm{~Hz}, 1 \mathrm{H}), 4.99$ (dd, 
$J=17.3,1.7 \mathrm{~Hz}, 1 \mathrm{H}), 5.66-5.80(\mathrm{~m}, 1 \mathrm{H}), 6.86(\mathrm{~d}, J=8.9 \mathrm{~Hz}, 2 \mathrm{H}), 7.05(\mathrm{~d}, J=9.0 \mathrm{~Hz}, 2 \mathrm{H}) .{ }^{13} \mathrm{C}$ $\operatorname{NMR}\left(75.5 \mathrm{MHz}, \mathrm{CDCl}_{3}\right) \delta 21.1\left(\mathrm{t},{ }^{3} J_{\mathrm{CF}}=3.7 \mathrm{~Hz}\right), 33.0,34.2\left(\mathrm{t},{ }^{2} J_{\mathrm{CF}}=23.9 \mathrm{~Hz}\right), 55.4,114.1$, $115.5,118.3\left(\mathrm{t},{ }^{1} J_{\mathrm{CF}}=246.4 \mathrm{~Hz}\right), 123.1,137.5,158.5 .{ }^{19} \mathrm{~F} \mathrm{NMR}\left(282.4 \mathrm{MHz}, \mathrm{CDCl}_{3}\right) \delta-99.18(\mathrm{t}$, $\left.J_{\mathrm{FH}}=16.5 \mathrm{~Hz}, 2 \mathrm{~F}\right)$. HRMS (EI) calcd for $\mathrm{C}_{14} \mathrm{H}_{16} \mathrm{ClF}_{2} \mathrm{NO}\left(\mathrm{M}^{+}\right): 287.0888$, found: 287.0847. Anal. calcd for $\mathrm{C}_{14} \mathrm{H}_{16} \mathrm{ClF}_{2} \mathrm{NO}: \mathrm{C}, 58.44 ; \mathrm{H}, 5.60 ; \mathrm{N}, 4.87$, found: $\mathrm{C}, 58.28 ; \mathrm{H}, 5.75 ; \mathrm{N}, 4.81$.

\section{Synthesis of ethyl (E)-6-chloro-5,5-difluoro-6-(4-methoxyphenylimino)-2-hexenoate, 5a.}<smiles>CCOC(=O)/C=C/CC(F)(F)/C(Cl)=N/P=O</smiles>

By means of General Procedure C, from $400 \mathrm{mg}(1.54 \mathrm{mmol})$ of $1 \mathbf{a},{ }^{2} 485 \mathrm{mg}$ of 5a were obtained as a colorless oil (95\% yield), as a mixture of $E / Z$ isomers in a 23:1 ratio determined by GC-MS. Data of the $E$ isomer: $R_{f}=0.10$ (hexane-diisopropyl ether, 7:1). ${ }^{1} \mathrm{H}$ NMR (300 MHz, $\left.\mathrm{CDCl}_{3}\right) \delta 1.23(\mathrm{t}, J=7.2 \mathrm{~Hz}, 3 \mathrm{H}), 3.14(\mathrm{tdd}, J=15.6,7.3,1.5 \mathrm{~Hz}, 2 \mathrm{H}), 3.76(\mathrm{~s}, 3 \mathrm{H}), 4.14(\mathrm{q}, J=$ $7.2 \mathrm{~Hz}, 2 \mathrm{H}), 5.99(\mathrm{~d}, J=15.6 \mathrm{~Hz}, 1 \mathrm{H}), 6.87(\mathrm{~d}, J=9.0 \mathrm{~Hz}, 2 \mathrm{H}), 6.91(\mathrm{dt}, J=15.6,7.4 \mathrm{~Hz}, 1 \mathrm{H})$, $7.10(\mathrm{~d}, J=9.0 \mathrm{~Hz}, 2 \mathrm{H}) .{ }^{13} \mathrm{C}$ NMR $\left(75.5 \mathrm{MHz}, \mathrm{CDCl}_{3}\right) \delta 14.2,37.9\left(\mathrm{t},{ }^{2} J_{\mathrm{CF}}=25.0 \mathrm{~Hz}\right), 55.5,60.6$, $114.1,116.6\left(\mathrm{t},{ }^{1} J_{\mathrm{CF}}=247.5 \mathrm{~Hz}\right), 123.5,127.1,136.2\left(\mathrm{t},{ }^{2} J_{\mathrm{CF}}=37.4 \mathrm{~Hz}\right), 136.5,137.3\left(\mathrm{t},{ }^{3} J_{\mathrm{CF}}=4.8\right.$ $\mathrm{Hz}), 158.8,165.6 .{ }^{19} \mathrm{~F}$ NMR $\left(282.4 \mathrm{MHz}, \mathrm{CDCl}_{3}\right) \delta-97.72\left(\mathrm{t}, J_{\mathrm{FH}}=15.5 \mathrm{~Hz}, 2 \mathrm{~F}\right)$. HRMS (EI) calcd for $\mathrm{C}_{15} \mathrm{H}_{16} \mathrm{ClF}_{2} \mathrm{NO}_{3}\left(\mathrm{M}^{+}\right)$: 331.0787, found: 331.0777. Anal. calcd for $\mathrm{C}_{15} \mathrm{H}_{16} \mathrm{ClF}_{2} \mathrm{NO}_{3}: \mathrm{C}$, $54.31 ; \mathrm{H}, 4.86 ; \mathrm{N}, 4.22$, found: $\mathrm{C}, 54.58 ; \mathrm{H}, 4.63 ; \mathrm{N}, 4.01$.

\footnotetext{
${ }^{2}$ Fustero, S.; Bartolomé, A.; Sanz-Cervera, J. F.; Sánchez-Roselló, M.; García Soler, J.; Ramírez de Arellano, C.; Simón Fuentes, A. Org. Lett. 2003, 5, 2523-2526.
} 
Synthesis of ethyl (E)-7-chloro-6,6-difluoro-7-(4-methoxyphenylimino)-2-heptenoate, 5 b.<smiles>CCOC(=O)/C=C/CCC(F)(F)/C(Cl)=N/P=N</smiles>

By means of General Procedure C, from $475 \mathrm{mg}(1.74 \mathrm{mmol})$ of $\mathbf{1 b}, 564 \mathrm{mg}$ of $5 \mathbf{b}$ were obtained as a colorless oil ( $94 \%$ yield), as a mixture of $E / Z$ isomers in a 46:1 ratio determined by GC-MS. Data of the $E$ isomer: $R_{f}=0.10$ (hexane-diisopropyl ether, $\left.7: 1\right) .{ }^{1} \mathrm{H}$ NMR $\left(300 \mathrm{MHz}, \mathrm{CDCl}_{3}\right) \delta$ $1.20(\mathrm{t}, J=7.2 \mathrm{~Hz}, 3 \mathrm{H}), 2.32-2.48(\mathrm{~m}, 4 \mathrm{H}), 3.75(\mathrm{~s}, 3 \mathrm{H}), 4.11(\mathrm{q}, J=7.2 \mathrm{~Hz}, 2 \mathrm{H}), 5.85(\mathrm{~d}, J=15.6$ $\mathrm{Hz}, 1 \mathrm{H}), 6.85(\mathrm{~d}, J=8.9 \mathrm{~Hz}, 2 \mathrm{H}), 6.91(\mathrm{dt}, J=15.6,6.3 \mathrm{~Hz}, 1 \mathrm{H}), 7.08(\mathrm{~d}, J=8.9 \mathrm{~Hz}, 2 \mathrm{H}) .{ }^{13} \mathrm{C}$ NMR (75.5 MHz, $\left.\mathrm{CDCl}_{3}\right) \delta 14.2,24.7\left(\mathrm{t},{ }^{3} J_{\mathrm{CF}}=4.6 \mathrm{~Hz}\right), 33.3\left(\mathrm{t},{ }^{2} J_{\mathrm{CF}}=23.9 \mathrm{~Hz}\right), 55.4,60.3$, 114.1, 117.6 (t, $\left.{ }^{1} J_{\mathrm{CF}}=247.0 \mathrm{~Hz}\right), 122.4,123.3,136.6,136.7\left(\mathrm{t},{ }^{2} J_{\mathrm{CF}}=37.4 \mathrm{~Hz}\right), 146.0,158.7$, 166.2. ${ }^{19} \mathrm{~F}$ NMR $\left(282.4 \mathrm{MHz}, \mathrm{CDCl}_{3}\right) \delta-99.40\left(\mathrm{t}, J_{\mathrm{FH}}=15.5 \mathrm{~Hz}, 2 \mathrm{~F}\right)$. HRMS (EI) calcd for $\mathrm{C}_{16} \mathrm{H}_{18} \mathrm{ClF}_{2} \mathrm{NO}_{3}\left(\mathrm{M}^{+}\right):$345.09439, found: 345.0937. Anal. calcd for $\mathrm{C}_{16} \mathrm{H}_{18} \mathrm{ClF}_{2} \mathrm{NO}_{3}$ : C, 55.58; H, 5.25; N, 4.05, found: C, 55.88; H, 5.43; N, 3.89.

\section{Synthesis of ethyl (E)-8-chloro-7,7-difluoro-8-(4-methoxyphenylimino)-2-octenoate, 5c.}<smiles>CCOC(=O)/C=C/CCCC(F)(F)/C(Cl)=N/P=N</smiles>

By means of General Procedure C, from $360 \mathrm{mg}(1.25 \mathrm{mmol})$ of 1c, $419 \mathrm{mg}$ of 5c were obtained as a colorless oil (93\% yield), as a mixture of $E / Z$ isomers in a 11:1 ratio determined by GC-MS. Data of the $E$ isomer: $R_{f}=0.10$ (hexane-diisopropyl ether, 7:1). ${ }^{1} \mathrm{H} \mathrm{NMR}\left(300 \mathrm{MHz}, \mathrm{CDCl}_{3}\right) \delta$ 
$1.21(\mathrm{t}, J=7.2 \mathrm{~Hz}, 3 \mathrm{H}), 1.70-1.75(\mathrm{~m}, 2 \mathrm{H}), 2.20-2.28(\mathrm{~m}, 4 \mathrm{H}), 3.75(\mathrm{~s}, 3 \mathrm{H}), 4.11$ (q, J= $7.2 \mathrm{~Hz}$ 2H), $5.80(\mathrm{dt}, J=15.6,1.5 \mathrm{~Hz}, 1 \mathrm{H}), 6.85$ (d, $J=9.0 \mathrm{~Hz}, 2 \mathrm{H}), 6.87$ (td, $J=15.6,6.8 \mathrm{~Hz}, 1 \mathrm{H}), 7.08$ $(\mathrm{d}, J=9.0 \mathrm{~Hz}, 2 \mathrm{H}) .{ }^{13} \mathrm{C} \mathrm{NMR}\left(75.5 \mathrm{MHz}, \mathrm{CDCl}_{3}\right) \delta 14.2,20.4\left(\mathrm{t},{ }^{3} J_{\mathrm{CF}}=4.0 \mathrm{~Hz}\right), 31.4,34.2(\mathrm{t}$, $\left.{ }^{2} J_{\mathrm{CF}}=23.9 \mathrm{~Hz}\right), 55.4,60.2,114.1,118.1\left(\mathrm{t},{ }^{1} J_{\mathrm{CF}}=246.7 \mathrm{~Hz}\right), 122.3,123.2,136.9,137.1\left(\mathrm{t},{ }^{2} J_{\mathrm{CF}}=\right.$ 37.4 Hz), 147.4, 158.6, 166.4. ${ }^{19} \mathrm{~F}$ NMR $\left(282.4 \mathrm{MHz}, \mathrm{CDCl}_{3}\right) \delta-98.95\left(\mathrm{t}, J_{\mathrm{FH}}=16.5 \mathrm{~Hz}, 2 \mathrm{~F}\right)$. HRMS (EI) calcd for $\mathrm{C}_{17} \mathrm{H}_{20} \mathrm{ClF}_{2} \mathrm{NO}_{3}\left(\mathrm{M}^{+}\right)$: 359.1099, found: 359.1166. Anal. calcd for $\mathrm{C}_{17} \mathrm{H}_{20} \mathrm{ClF}_{2} \mathrm{NO}_{3}: \mathrm{C}, 56.75 ; \mathrm{H}, 5.60 ; \mathrm{N}, 3.89$, found: $\mathrm{C}, 56.90 ; \mathrm{H}, 5.73 ; \mathrm{N}, 3.81$.

\section{Synthesis of ethyl 6-chloro-5,5-difluoro-6-(4-methoxyphenylimino)-hexanoate, 6a.}

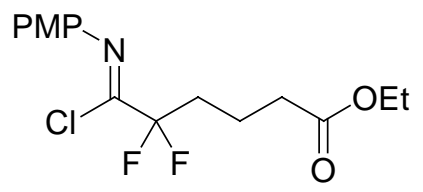

By means of General Procedure D, from $300 \mathrm{mg}(0.90 \mathrm{mmol})$ of $\mathbf{5 a}, 201 \mathrm{mg}$ of $\mathbf{6 a}$ were obtained as a colorless oil (68\% yield). $R_{f}=0.40$ (hexane/EtOAc, $\left.10: 1\right) .{ }^{1} \mathrm{H}$ NMR $\left(300 \mathrm{MHz}, \mathrm{CDCl}_{3}\right) \delta$ $1.18(\mathrm{t}, J=7.2 \mathrm{~Hz}, 3 \mathrm{H}), 1.86-1.91(\mathrm{~m}, 2 \mathrm{H}), 2.25-2.38(\mathrm{~m}, 4 \mathrm{H}), 3.75$ (s, 3H), 4.07 (q, $J=7.2 \mathrm{~Hz}$, 2H), $6.86(\mathrm{~d}, J=9.1 \mathrm{~Hz}, 2 \mathrm{H}), 7.07(\mathrm{~d}, J=9.1 \mathrm{~Hz}, 2 \mathrm{H}) .{ }^{13} \mathrm{C} \mathrm{NMR}\left(75.5 \mathrm{MHz}, \mathrm{CDCl}_{3}\right) \delta 14.2,17.5$ $\left(\mathrm{t},{ }^{3} J_{\mathrm{CF}}=4.3 \mathrm{~Hz}\right), 33.5,34.1\left(\mathrm{t},{ }^{2} J_{\mathrm{CF}}=23.9 \mathrm{~Hz}\right), 55.4,60.5,114.1,118.0\left(\mathrm{t},{ }^{1} J_{\mathrm{CF}}=246.4 \mathrm{~Hz}\right)$, 123.2, 136.9, $137.2\left(\mathrm{t},{ }^{2} J_{\mathrm{CF}}=37.1 \mathrm{~Hz}\right), 158.6,172.7 .{ }^{19} \mathrm{~F} \mathrm{NMR}\left(282.4 \mathrm{MHz}, \mathrm{CDCl}_{3}\right) \delta-99.32(\mathrm{t}$, $J_{\mathrm{FH}}=16.5 \mathrm{~Hz}, 2 \mathrm{~F}$ ). HRMS (EI) calcd for $\mathrm{C}_{15} \mathrm{H}_{18} \mathrm{ClF}_{2} \mathrm{NO}_{3}\left(\mathrm{M}^{+}\right)$: 333.0943, found: 333.0886 . Anal. calcd for $\mathrm{C}_{15} \mathrm{H}_{18} \mathrm{ClF}_{2} \mathrm{NO}_{3}$ : C, 53.98; H, 5.44; N, 4.20, found: C, 54.13; H, 5.56; N, 4.29. 
Synthesis of ethyl 7-chloro-6,6-difluoro-7-(4-methoxyphenylimino)-heptanoate, 6 b.<smiles>CCOC(=O)CCCCC(F)(F)/C(Cl)=N/P=NP</smiles>

By means of General Procedure D, from $120 \mathrm{mg}(0.35 \mathrm{mmol})$ of $5 \mathbf{b}, 86 \mathrm{mg}$ of $\mathbf{6 b}$ were obtained as a colorless oil $\left(72 \%\right.$ yield). $R_{f}=0.30$ (hexane/EtOAc, $\left.10: 1\right) .{ }^{1} \mathrm{H}$ NMR $\left(300 \mathrm{MHz}, \mathrm{CDCl}_{3}\right) \delta$ $1.18(\mathrm{t}, J=7.2 \mathrm{~Hz}, 3 \mathrm{H}), 1.57-1.70(\mathrm{~m}, 4 \mathrm{H}), 2.23-2.30(\mathrm{~m}, 4 \mathrm{H}), 3.76(\mathrm{~s}, 3 \mathrm{H}), 4.06(\mathrm{q}, J=7.2 \mathrm{~Hz}$ 2H), $6.86(\mathrm{~d}, J=9.0 \mathrm{~Hz}, 2 \mathrm{H}), 7.06(\mathrm{~d}, J=9.0 \mathrm{~Hz}, 2 \mathrm{H}) .{ }^{13} \mathrm{C} \mathrm{NMR}\left(75.5 \mathrm{MHz}, \mathrm{CDCl}_{3}\right) \delta 14.2,21.4$ $\left(\mathrm{t},{ }^{3} J_{\mathrm{CF}}=4.0 \mathrm{~Hz}\right), 24.5,33.9,34.5\left(\mathrm{t},{ }^{2} J_{\mathrm{CF}}=23.9 \mathrm{~Hz}\right), 55.4,60.3,114.1,118.1\left(\mathrm{t},{ }^{1} J_{\mathrm{CF}}=246.4 \mathrm{~Hz}\right)$, 123.1, 137.0, $137.4\left(\mathrm{t},{ }^{2} J_{\mathrm{CF}}=37.1 \mathrm{~Hz}\right), 158.5,173.2 .{ }^{19} \mathrm{~F}$ NMR $\left(282.4 \mathrm{MHz}, \mathrm{CDCl}_{3}\right) \delta-99.06(\mathrm{t}$, $\left.J_{\mathrm{FH}}=16.5 \mathrm{~Hz}, 2 \mathrm{~F}\right)$. HRMS (EI) calcd for $\mathrm{C}_{16} \mathrm{H}_{20} \mathrm{ClF}_{2} \mathrm{NO}_{3}\left(\mathrm{M}^{+}\right)$: 347.1099, found: 347.1098 . Anal. calcd for $\mathrm{C}_{16} \mathrm{H}_{20} \mathrm{ClF}_{2} \mathrm{NO}_{3}$ : C, 55.26; H, 5.80; N, 4.03, found: C, 55.07; H, 5.68; N, 4.12.

\section{Synthesis of ethyl 8-chloro-7,7-difluoro-8-(4-methoxyphenylimino)-octanoate, 6c.}

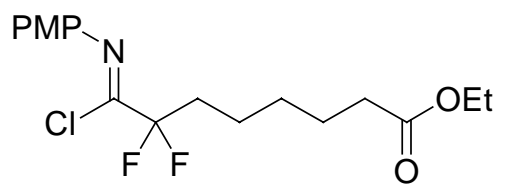

By means of General Procedure D, from $365 \mathrm{mg}(1.01 \mathrm{mmol})$ of 5c, $277 \mathrm{mg}$ of 6c were obtained as a colorless oil (75\% yield). $R_{f}=0.45$ (hexane/EtOAc, $\left.7: 1\right) .{ }^{1} \mathrm{H}$ NMR $\left(300 \mathrm{MHz}, \mathrm{CDCl}_{3}\right) \delta 1.15$ $(\mathrm{t}, J=7.1 \mathrm{~Hz}, 3 \mathrm{H}), 1.23-1.36(\mathrm{~m}, 2 \mathrm{H}), 1.48-1.61(\mathrm{~m}, 4 \mathrm{H}), 2.15-2.24(\mathrm{~m}, 4 \mathrm{H}), 3.71(\mathrm{~s}, 3 \mathrm{H}), 4.02$ $(\mathrm{dq}, J=7.1,1.9 \mathrm{~Hz}, 2 \mathrm{H}), 6.83(\mathrm{~d}, J=9.0 \mathrm{~Hz}, 2 \mathrm{H}), 7.03(\mathrm{~d}, J=9.0 \mathrm{~Hz}, 2 \mathrm{H}) ;{ }^{13} \mathrm{C} \mathrm{NMR}(75.5 \mathrm{MHz}$, $\left.\mathrm{CDCl}_{3}\right) \delta 14.2,21.5\left(\mathrm{t},{ }^{4} J_{\mathrm{CF}}=4.0 \mathrm{~Hz}\right), 24.6\left(\mathrm{t},{ }^{3} J_{\mathrm{CF}}=12.1 \mathrm{~Hz}\right), 28.7\left(\mathrm{t},{ }^{5} J_{\mathrm{CF}}=3.4 \mathrm{~Hz}\right), 34.1,34.7$ 
$\left(\mathrm{t},{ }^{2} J_{\mathrm{CF}}=23.6 \mathrm{~Hz}\right), 55.4,60.2,114.1,118.2\left(\mathrm{t},{ }^{1} J_{\mathrm{CF}}=246.7 \mathrm{~Hz}\right), 123.1,137.1,137.5\left(\mathrm{t},{ }^{2} J_{\mathrm{CF}}=37.0\right.$ $\mathrm{Hz}), 158.5,173.6 ;{ }^{19} \mathrm{~F}$ NMR $\left(282.4 \mathrm{MHz}, \mathrm{CDCl}_{3}\right) \delta-99.24\left(\mathrm{tt},{ }^{1} J_{\mathrm{FH}}=16.5,{ }^{2} J_{\mathrm{FH}}=5.2 \mathrm{~Hz}, 2 \mathrm{~F}\right)$. HRMS (EI) calcd for $\mathrm{C}_{17} \mathrm{H}_{22} \mathrm{ClF}_{2} \mathrm{NO}_{3}\left(\mathrm{M}^{+}\right)$: 361.1256, found: 361.1250. Anal. calcd for $\mathrm{C}_{17} \mathrm{H}_{22} \mathrm{ClF}_{2} \mathrm{NO}_{3}: \mathrm{C}, 56.43 ; \mathrm{H}, 6.13 ; \mathrm{N}, 3.87$, found: $\mathrm{C}, 56.29 ; \mathrm{H}, 6.21 ; \mathrm{N}, 3.96$.

\section{Synthesis of 3,3-difluoro-1-(ethoxycarbonyl)-2-(4-methoxyphenylamino)-1-cyclopentene,}

7a.

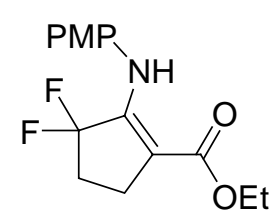

By means of General Procedure E, from $200 \mathrm{mg}(0.60 \mathrm{mmol})$ of $\mathbf{6 a}, 143 \mathrm{mg}$ of $7 \mathbf{a}$ were obtained as a yellow solid (80\% yield). $R_{f}=0.34$ (hexane/EtOAc, 10:1). Mp: $58-61{ }^{\circ} \mathrm{C} .{ }^{1} \mathrm{H}$ NMR $(300$ $\left.\mathrm{MHz}, \mathrm{CDCl}_{3}\right) \delta 1.25(\mathrm{t}, J=7.1 \mathrm{~Hz}, 3 \mathrm{H}), 2.17-2.31(\mathrm{~m}, 2 \mathrm{H}), 2.48-2.52(\mathrm{~m}, 2 \mathrm{H}), 3.72(\mathrm{~s}, 3 \mathrm{H}), 4.16$ (q, $J=7.1 \mathrm{~Hz}, 2 \mathrm{H}), 6.76$ (d, $J=9.0 \mathrm{~Hz}, 2 \mathrm{H}), 7.10(\mathrm{~d}, J=9.0 \mathrm{~Hz}, 2 \mathrm{H}), 8.65(\mathrm{br}, 1 \mathrm{H}) .{ }^{13} \mathrm{C}$ NMR $\left(75.5 \mathrm{MHz}, \mathrm{CDCl}_{3}\right) \delta 14.5,22.7\left(\mathrm{t},{ }^{3} J_{\mathrm{CF}}=2.7 \mathrm{~Hz}\right), 33.3\left(\mathrm{t},{ }^{2} J_{\mathrm{CF}}=24.4 \mathrm{~Hz}\right), 55.4,59.8,101.0(\mathrm{t}$, $\left.{ }^{3} J_{\mathrm{CF}}=7.5 \mathrm{~Hz}\right), 113.9,125.6,128.9\left(\mathrm{t},{ }^{1} J_{\mathrm{CF}}=249.0 \mathrm{~Hz}\right), 131.6,149.6\left(\mathrm{t},{ }^{2} J_{\mathrm{CF}}=23.3 \mathrm{~Hz}\right), 157.2$, 167.8. ${ }^{19} \mathrm{~F}$ NMR $\left(282.4 \mathrm{MHz}, \mathrm{CDCl}_{3}\right) \delta-87.82\left(\mathrm{tt},{ }^{1} J_{\mathrm{FH}}=15.9 \mathrm{~Hz},{ }^{2} J_{\mathrm{FH}}=5.1 \mathrm{~Hz}, 2 \mathrm{~F}\right)$. HRMS (EI) calcd for $\mathrm{C}_{15} \mathrm{H}_{17} \mathrm{~F}_{2} \mathrm{NO}_{3}\left(\mathrm{M}^{+}\right)$: 297.1177, found: 297.1148. Anal. calcd for $\mathrm{C}_{15} \mathrm{H}_{17} \mathrm{~F}_{2} \mathrm{NO}_{3}$ : $\mathrm{C}$, 60.60; H, 5.76; N, 4.71, found: C, 60.46; H, 5.62; N, 4.57. 
Synthesis of 3,3-difluoro-1-(ethoxycarbonyl)-2-(4-methoxyphenylamino)-1-cyclohexene, 7b.

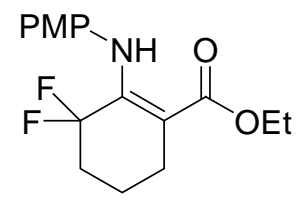

By means of General Procedure E, from $66 \mathrm{mg}(0.19 \mathrm{mmol})$ of $\mathbf{6 b}, 44 \mathrm{mg}$ of $7 \mathbf{b}$ were obtained as a yellow solid (75\% yield). $R_{f}=0.20$ (hexane/EtOAc, 20:1). Mp: 66-68 ${ }^{\circ} \mathrm{C} .{ }^{1} \mathrm{H} \mathrm{NMR}(300 \mathrm{MHz}$, $\left.\mathrm{CDCl}_{3}\right) \delta 1.22(\mathrm{t}, J=7.2 \mathrm{~Hz}, 3 \mathrm{H}), 1.70-1.74(\mathrm{~m}, 2 \mathrm{H}), 1.95-1.99(\mathrm{~m}, 2 \mathrm{H}), 2.35-2.38(\mathrm{~m}, 2 \mathrm{H}), 3.71$ (s, 3H), 4.11 (q, J=7.2 Hz, 2H), 6.74 (d, J=8.9 Hz, 2H), 7.07 (d, J=8.9 Hz, 2H), 9.94 (br, 1H). ${ }^{13} \mathrm{C}$ NMR $\left(75.5 \mathrm{MHz}, \mathrm{CDCl}_{3}\right) \delta 14.3,18.4\left(\mathrm{t},{ }^{3} J_{\mathrm{CF}}=4.9 \mathrm{~Hz}\right), 24.0,34.2\left(\mathrm{t},{ }^{2} J_{\mathrm{CF}}=23.6 \mathrm{~Hz}\right), 55.3$, $60.0,98.3\left(\mathrm{t},{ }^{3} J_{\mathrm{CF}}=6.0 \mathrm{~Hz}\right), 113.5,118.8\left(\mathrm{t},{ }^{1} J_{\mathrm{CF}}=243.2 \mathrm{~Hz}\right), 127.5,133.7,147.7\left(\mathrm{t},{ }^{2} J_{\mathrm{CF}}=23.0\right.$ $\mathrm{Hz}), 157.3,170.2 .{ }^{19} \mathrm{~F}$ NMR $\left(282.4 \mathrm{MHz}, \mathrm{CDCl}_{3}\right) \delta-89.67\left(\mathrm{t}, J_{\mathrm{FH}}=14.4 \mathrm{~Hz}, 2 \mathrm{~F}\right) . \mathrm{HRMS}(\mathrm{EI})$ calcd for $\mathrm{C}_{16} \mathrm{H}_{19} \mathrm{~F}_{2} \mathrm{NO}_{3}\left(\mathrm{M}^{+}\right)$: 311.1333, found: 311.1408. Anal. calcd for $\mathrm{C}_{16} \mathrm{H}_{19} \mathrm{~F}_{2} \mathrm{NO}_{3}$ : $\mathrm{C}$, 61.73; H, 6.15; N, 4.50, found: C, 61.59; H, 6.02; N, 4.58.

\section{Synthesis of 3,3-difluoro-1-(ethoxycarbonyl)-2-(4-methoxyphenylamino)-1-cycloheptene,} 7c.

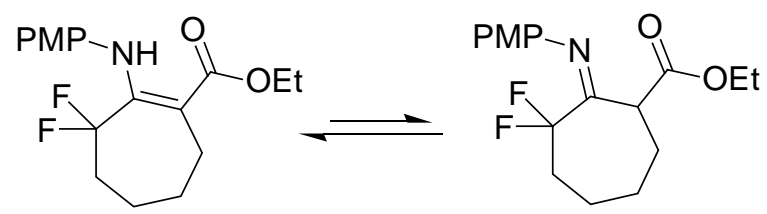

By means of General Procedure E, from $92 \mathrm{mg}(0.25 \mathrm{mmol})$ of $6 \mathbf{c}, 7 \mathbf{c}$ was obtained as a mixture of enamino (20 mg, 24\% yield) and imino forms (13 mg, 16\% yield), both as yellow oils. Data of the enamino form: $R_{f}=0.55$ (hexane/EtOAc, $\left.10: 1\right) .{ }^{1} \mathrm{H} \mathrm{NMR}\left(300 \mathrm{MHz}, \mathrm{CDCl}_{3}\right) \delta 1.23(\mathrm{t}, \mathrm{J}=$ 
$7.2 \mathrm{~Hz}, 3 \mathrm{H}), 1.54(\mathrm{br}, 2 \mathrm{H}), 1.68-1.77(\mathrm{~m}, 2 \mathrm{H}), 1.91-2.05(\mathrm{~m}, 2 \mathrm{H}), 2.34-2.39(\mathrm{~m}, 2 \mathrm{H}), 3.72(\mathrm{~s}$, 3H), 4.12 (q, $J=7.2 \mathrm{~Hz}, 2 \mathrm{H}), 6.75(\mathrm{~d}, J=8.9 \mathrm{~Hz}, 2 \mathrm{H}), 7.08$ (d, $J=8.9 \mathrm{~Hz}, 2 \mathrm{H}), 9.96$ (br, $1 \mathrm{H}) .{ }^{13} \mathrm{C}$ $\operatorname{NMR}\left(75.5 \mathrm{MHz}, \mathrm{CDCl}_{3}\right) \delta 14.4,18.4\left(\mathrm{t},{ }^{3} J_{\mathrm{CF}}=4.6 \mathrm{~Hz}\right), 24.0,34.2\left(\mathrm{t},{ }^{2} J_{\mathrm{CF}}=23.6 \mathrm{~Hz}\right), 55.3,60.0$, $98.3\left(\mathrm{t},{ }^{3} J_{\mathrm{CF}}=9.7 \mathrm{~Hz}\right), 113.5,118.8\left(\mathrm{t},{ }^{1} J_{\mathrm{CF}}=243.2 \mathrm{~Hz}\right), 127.5,133.7,147.7\left(\mathrm{t},{ }^{2} J_{\mathrm{CF}}=23.0 \mathrm{~Hz}\right)$, 157.2, 170.3. ${ }^{19} \mathrm{~F}$ NMR $\left(282.4 \mathrm{MHz}, \mathrm{CDCl}_{3}\right) \delta-89.66\left(\mathrm{t}, J_{\mathrm{FH}}=14.4 \mathrm{~Hz}, 2 \mathrm{~F}\right)$. HRMS (EI) calcd for $\mathrm{C}_{17} \mathrm{H}_{21} \mathrm{~F}_{2} \mathrm{NO}_{3}\left(\mathrm{M}^{+}\right)$: 325.1490 , found: 325.1481. Data of the imino form: $R_{f}=0.35$ (hexane/EtOAc, 10:1). ${ }^{1} \mathrm{H}$ NMR (300 MHz, $\left.\mathrm{CDCl}_{3}\right) \delta$ 0.74-0.90 (m, 1H), 0.96-1.14 (m, 1H), $1.22(\mathrm{t}, J=7.1 \mathrm{~Hz}, 3 \mathrm{H}), 1.43-1.60(\mathrm{~m}, 1 \mathrm{H}), 1.73-1.88(\mathrm{~m}, 2 \mathrm{H}), 1.89-2.15(\mathrm{~m}, 2 \mathrm{H}), 2.17-2.32(\mathrm{~m}$, 1H), $3.68(\mathrm{dd}, J=8.7,2.6 \mathrm{~Hz}, 1 \mathrm{H}), 3.73(\mathrm{~s}, 3 \mathrm{H}), 4.05-4.23(\mathrm{~m}, 2 \mathrm{H}), 6.71$ (d, $J=8.9 \mathrm{~Hz}, 2 \mathrm{H}), 6.82$ $(\mathrm{d}, J=8.9 \mathrm{~Hz}, 2 \mathrm{H}) .{ }^{19} \mathrm{~F}$ NMR $\left(282.4 \mathrm{MHz}, \mathrm{CDCl}_{3}\right) \delta-92.03\left(\mathrm{dd}, J_{\mathrm{FF}}=242.7 \mathrm{~Hz}, J_{\mathrm{FH}}=12.3 \mathrm{~Hz}\right.$, 1F), $-109.51\left(\mathrm{ddt}, J_{\mathrm{FF}}=242.5 \mathrm{~Hz}, J_{\mathrm{FH}}=34.1,3.0 \mathrm{~Hz}, 1 \mathrm{~F}\right)$.

Synthesis of cis-( \pm )-1,1-difluoro-3-(ethoxycarbonyl)-2-(4-methoxyphenylamino)cyclopentane, $( \pm)-8 \mathrm{a}$.

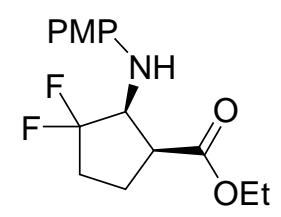

By means of General Procedure F, from $42 \mathrm{mg}(0.14 \mathrm{mmol})$ of $7 \mathbf{a}, 35 \mathrm{mg}$ of $\mathbf{8 a}$ were obtained as a yellow oil (83\% yield). $R_{f}=0.30$ (hexane/EtOAc, 7:1). ${ }^{1} \mathrm{H}$ NMR $\left(300 \mathrm{MHz}, \mathrm{CDCl}_{3}\right) \delta 1.08(\mathrm{t}$, $J=7.2 \mathrm{~Hz}, 3 \mathrm{H}), 1.92-2.30(\mathrm{~m}, 4 \mathrm{H}), 3.23(\mathrm{q}, J=7.3 \mathrm{~Hz}, 1 \mathrm{H}), 3.67(\mathrm{~s}, 3 \mathrm{H}), 3.97-4.05(\mathrm{~m}, 3 \mathrm{H}), 6.59$ $(\mathrm{d}, J=9.0 \mathrm{~Hz}, 2 \mathrm{H}), 6.70(\mathrm{~d}, J=9.0 \mathrm{~Hz}, 2 \mathrm{H}) .{ }^{13} \mathrm{C} \mathrm{NMR}\left(75.5 \mathrm{MHz}, \mathrm{CDCl}_{3}\right) \delta 14.0,22.6\left(\mathrm{t},{ }^{3} J_{\mathrm{CF}}=\right.$ $4.0 \mathrm{~Hz}), 32.3\left(\mathrm{t},{ }^{2} J_{\mathrm{CF}}=24.7 \mathrm{~Hz}\right), 44.7\left(\mathrm{t},{ }^{3} J_{\mathrm{CF}}=4.9 \mathrm{~Hz}\right), 55.7,61.0,61.2\left(\mathrm{dd},{ }^{2} J_{\mathrm{CF}}=29.6,21.0 \mathrm{~Hz}\right)$, 
$114.8,115.2,129.2\left(\mathrm{dd},{ }^{1} J_{\mathrm{CF}}=255.3,251.1 \mathrm{~Hz}\right), 140.7,152.8,172.1 .{ }^{19} \mathrm{~F}$ NMR $(282.4 \mathrm{MHz}$, $\left.\mathrm{CDCl}_{3}\right) \delta-97.60\left(\mathrm{ddt}, J_{\mathrm{FF}}=230.4 \mathrm{~Hz}, J_{\mathrm{FH}}=26.8,10.3 \mathrm{~Hz}, 1 \mathrm{~F}\right),-107.70\left(\mathrm{ddt}, J_{\mathrm{FF}}=229.9 \mathrm{~Hz}, J_{\mathrm{FH}}=\right.$ 24.7, $10.3 \mathrm{~Hz}, 1 \mathrm{~F})$. HRMS (EI) calcd for $\mathrm{C}_{15} \mathrm{H}_{19} \mathrm{~F}_{2} \mathrm{NO}_{3}\left(\mathrm{M}^{+}\right)$: 299.1333, found: 299.1342. Anal. calcd for $\mathrm{C}_{15} \mathrm{H}_{19} \mathrm{~F}_{2} \mathrm{NO}_{3}$ : C, 60.19; $\mathrm{H}, 6.40 ; \mathrm{N}, 4.68$, found: $\mathrm{C}, 60.34 ; \mathrm{H}, 6.57 ; \mathrm{N}, 4.59$.

Synthesis of cis-( \pm )-1,1-difluoro-3-(ethoxycarbonyl)-2-(4-methoxyphenylamino)cyclohexane, $( \pm)-8 b$.

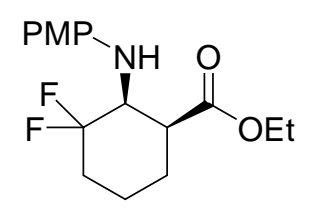

By means of General Procedure F, from $34 \mathrm{mg}(0.11 \mathrm{mmol})$ of $\mathbf{7 b}, 30 \mathrm{mg}$ of $\mathbf{8 b}$ were obtained as a yellow solid (87\% yield). $R_{f}=0.20$ (hexane/diisopropyl ether, $1: 1$ ). Mp: $79-81{ }^{\circ} \mathrm{C} .{ }^{1} \mathrm{H}$ NMR $\left(300 \mathrm{MHz}, \mathrm{CDCl}_{3}\right) \delta 0.95(\mathrm{t}, J=7.2 \mathrm{~Hz}, 3 \mathrm{H}), 1.19-1.97(\mathrm{~m}, 7 \mathrm{H}), 2.82-2.87(\mathrm{~m}, 1 \mathrm{H}), 3.66(\mathrm{~s}, 3 \mathrm{H})$, 3.90-3.98 (m, 1H), $3.92(\mathrm{q}, J=7.1 \mathrm{~Hz}, 2 \mathrm{H}), 6.61(\mathrm{~d}, J=9.0 \mathrm{~Hz}, 2 \mathrm{H}), 6.68(\mathrm{~d}, J=9.0 \mathrm{~Hz}, 2 \mathrm{H}) .{ }^{13} \mathrm{C}$ NMR $\left(100 \mathrm{MHz}, \mathrm{CDCl}_{3}\right) \delta 13.9,20.1\left(\mathrm{t},{ }^{3} J_{\mathrm{CF}}=9.8 \mathrm{~Hz}\right), 21.3,29.2\left(\mathrm{t},{ }^{2} J_{\mathrm{CF}}=23.2 \mathrm{~Hz}\right), 44.1(\mathrm{t}$, $\left.{ }^{3} J_{\mathrm{CF}}=5.5 \mathrm{~Hz}\right), 55.7,57.4\left(\mathrm{dd},{ }^{2} J_{\mathrm{CF}}=32.9,22.6 \mathrm{~Hz}\right), 60.9,114.6,115.6,123.3\left(\mathrm{dd},{ }^{1} J_{\mathrm{CF}}=249.9\right.$, $241.9 \mathrm{~Hz}), 141.4,152.9,172.0 .{ }^{19} \mathrm{~F}$ NMR $\left(282.4 \mathrm{MHz}, \mathrm{CDCl}_{3}\right) \delta-101.76\left(\mathrm{~d}, J_{\mathrm{FF}}=239.2 \mathrm{~Hz}, 1 \mathrm{~F}\right)$, $-105.04\left(\mathrm{dm}, J_{\mathrm{FF}}=239.2 \mathrm{~Hz}, 1 \mathrm{~F}\right)$. HRMS (EI) calcd for $\mathrm{C}_{16} \mathrm{H}_{21} \mathrm{~F}_{2} \mathrm{NO}_{3}\left(\mathrm{M}^{+}\right): 313.1489$, found: 313.1437. Anal. calcd for $\mathrm{C}_{16} \mathrm{H}_{21} \mathrm{~F}_{2} \mathrm{NO}_{3}: \mathrm{C}, 61.33 ; \mathrm{H}, 6.76 ; \mathrm{N}, 4.47$, found: $\mathrm{C}, 61.22 ; \mathrm{H}, 6.80 ; \mathrm{N}$, 4.54 . 
Synthesis of cis-( \pm )-1,1-difluoro-3-(ethoxycarbonyl)-2-(4-methoxyphenylamino)cycloheptane, $( \pm)-8 c$.

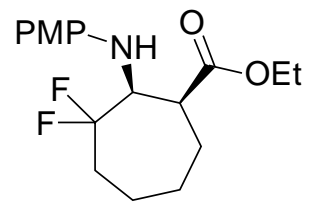

By means of General Procedure F, from $50 \mathrm{mg}(0.15 \mathrm{mmol})$ of 7c, $41 \mathrm{mg}$ of 8c were obtained as a yellow solid (82\% yield). $R_{f}=0.20$ (hexane/diisopropyl ether, 1:1). Its physical and spectroscopic data matched those previously reported. ${ }^{2}$

Synthesis of $\quad(+)-(E)-\left(1^{\prime} R, 2 ' S, 5 ’ R\right)-8$ '-phenylmenthyl 6-chloro-5,5-difluoro-6-(4methoxyphenylimino)-2-hexenoate, $(+)-5 d$.<smiles>C[C@H]1CC[C@@H](C(C)(C)c2ccccc2)[C@H](OC(=O)/C=C/CC(F)(F)/C(Cl)=N/[PH]#N)C1</smiles>

By means of General Procedure C, from $363 \mathrm{mg}(1.40 \mathrm{mmol})$ of 1a and (+)-8-phenylmenthyl acrylate $^{3}(800 \mathrm{mg}, 2.79 \mathrm{mmol}), 550 \mathrm{mg}$ of $5 \mathbf{d}$ were obtained as a colorless oil (76\% yield), as a mixture of $E / Z$ isomers in a $12: 1$ ratio determined by GC-MS. Data of the $E$ isomer: $R_{f}=0.15$ (hexane/EtOAc, 15:1). $[\alpha]_{\mathrm{D}}^{25}=+3.8\left(\mathrm{c}_{1.1}, \mathrm{CHCl}_{3}\right) .{ }^{1} \mathrm{H} \mathrm{NMR}\left(300 \mathrm{MHz}, \mathrm{CDCl}_{3}\right): \delta 0.79(\mathrm{~d}, J=$ $6.4 \mathrm{~Hz}, 3 \mathrm{H}), 0.90(\mathrm{q}, J=12.0 \mathrm{~Hz}, 2 \mathrm{H}), 1.04(\mathrm{qd}, J=13.2,3.0 \mathrm{~Hz}, 1 \mathrm{H}), 1.13(\mathrm{~s}, 3 \mathrm{H}), 1.21(\mathrm{~s}, 3 \mathrm{H})$, 1.36-1.43 (m, 1H), 1.53-1.68 (m, 2H), 1.82 (br d, $J=11.7 \mathrm{~Hz}, 1 \mathrm{H}), 1.98(\mathrm{ddd}, J=12.4,10.9,3.4$ Hz, 1H), 2.97 (tdd, $J=15.8,7.2,1.1 \mathrm{~Hz}, 2 \mathrm{H}), 3.75(\mathrm{~s}, 3 \mathrm{H}), 4.78(\mathrm{td}, J=10.5,4.5 \mathrm{~Hz}, 1 \mathrm{H}), 5.37$

\footnotetext{
${ }^{3}$ Whitesell, J. K.; Bhattacharya, A.; Buchanan, C. M.; Chen, H. H.; Deyo, D.; James, D.; Liu, C.-L.; Minton, M. A. Tetrahedron 1986, 42, 2993-3001.
} 
(d, $J=15.8 \mathrm{~Hz}, 1 \mathrm{H}), 6.44(\mathrm{dt}, J=15.5,7.5 \mathrm{~Hz}, 1 \mathrm{H}), 6.85(\mathrm{~d}, J=9.0 \mathrm{~Hz}, 2 \mathrm{H}), 7.00-7.20(\mathrm{~m}, 5 \mathrm{H})$, $7.09(\mathrm{~d}, J=9.0 \mathrm{~Hz}, 2 \mathrm{H}) .{ }^{13} \mathrm{C}$ NMR $\left(75.5 \mathrm{MHz}, \mathrm{CDCl}_{3}\right): \delta 21.8,24.7,26.5,28.0,31.2,34.5,37.7$ $\left(\mathrm{t},{ }^{2} J_{\mathrm{CF}}=25.0 \mathrm{~Hz}\right), 39.6,41.6,50.5,55.4,74.5,114.1,116.6\left(\mathrm{t},{ }^{1} J_{\mathrm{CF}}=247.8 \mathrm{~Hz}\right), 123.5,124.8$, 125.4, 127.3, 127.9, $136.4\left(\mathrm{t},{ }^{2} J_{\mathrm{CF}}=18.7 \mathrm{~Hz}\right), 136.5,136.6,151.6,158.8,164.7 .{ }^{19} \mathrm{~F}$ NMR $(282.4$ $\left.\mathrm{MHz}, \mathrm{CDCl}_{3}\right): \delta-97.74\left(\mathrm{t}, J_{\mathrm{FH}}=15.6 \mathrm{~Hz}, 2 \mathrm{~F}\right) . \mathrm{HRMS}(\mathrm{EI})$ calcd for $\mathrm{C}_{29} \mathrm{H}_{34} \mathrm{ClF}_{2} \mathrm{NO}_{3}\left(\mathrm{M}^{+}\right)$: 517.2195; found: 517.2209.

\section{Synthesis of $\quad(+)-(E)-\left(1^{\prime} R, 2^{\prime} S, 5^{\prime} R\right)-8$ '-phenylmenthyl 6-chloro-5,5-difluoro-6-(4-} methoxyphenylimino)hexanoate, (+)-6d.

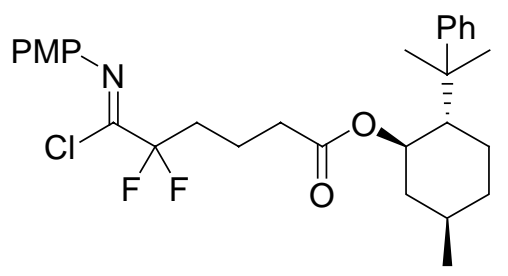

By means of General Procedure D, from $830 \mathrm{mg}(1.60 \mathrm{mmol})$ of $5 \mathbf{d}, 785 \mathrm{mg}$ of $\mathbf{6 d}$ were obtained as a colorless oil (94\% yield). $R_{f}=0.15$ (hexane/EtOAc, $\left.15: 1\right) .[\alpha]^{25}=+8.1(c 1.0$, $\left.\mathrm{CHCl}_{3}\right) .{ }^{1} \mathrm{H}$ NMR $\left(300 \mathrm{MHz}, \mathrm{CDCl}_{3}\right): \delta 0.79(\mathrm{~d}, J=6.4 \mathrm{~Hz}, 3 \mathrm{H}), 0.88(\mathrm{q}, J=12.1 \mathrm{~Hz}, 2 \mathrm{H}), 1.06$ (qd, $J=12.8,3.0 \mathrm{~Hz}, 1 \mathrm{H}), 1.12(\mathrm{~s}, 3 \mathrm{H}), 1.23(\mathrm{~s}, 3 \mathrm{H}), 1.35-1.41(\mathrm{~m}, 1 \mathrm{H}), 1.55-1.79(\mathrm{~m}, 7 \mathrm{H}), 1.92-$ 2.35 (m, 3H), 3.75 (s, 3H), 4.76 (td, $J=10.7,4.5 \mathrm{~Hz}, 1 \mathrm{H}), 6.86(\mathrm{~d}, J=9.0 \mathrm{~Hz}, 2 \mathrm{H}), 7.07$ (d, $J=$ $8.7 \mathrm{~Hz}, 2 \mathrm{H}), 7.18-7.22(\mathrm{~m}, 5 \mathrm{H}) .{ }^{13} \mathrm{C}$ NMR $\left(75.5 \mathrm{MHz}, \mathrm{CDCl}_{3}\right): \delta 17.2,21.8,23.9,26.4,28.8$, 31.2, 33.3, 34.1 (t, $\left.{ }^{2} J_{\mathrm{CF}}=23.9 \mathrm{~Hz}\right), 34.5,39.5,41.7,50.2,55.4,74.0,114.1,118.0\left(\mathrm{t},{ }^{1} J_{\mathrm{CF}}=246.7\right.$ $\mathrm{Hz}), 123.1,125.0,125.3,127.9,137.0,137.2\left(\mathrm{t},{ }^{2} J_{\mathrm{CF}}=37.0 \mathrm{~Hz}\right), 151.8,158.5,171.9 .{ }^{19} \mathrm{~F} \mathrm{NMR}$ (282.4 MHz, $\mathrm{CDCl}_{3}$ ): $\delta$-99.39 (br, 2F). HRMS (EI) calcd for $\mathrm{C}_{29} \mathrm{H}_{36} \mathrm{ClF}_{2} \mathrm{NO}_{3}\left(\mathrm{M}^{+}\right)$: 519.2352; found: 519.2324 . 
Synthesis of $\quad(+)-\left(1^{\prime} R, 2^{\prime} S, 5^{\prime} R\right)-3,3-d i f l u o r o-1-\left(8^{\prime}-p h e n y l m e n t h y l o x y c a r b o n y l\right)-2-(4-$ methoxyphenylamino)-1-cyclopentene, $(+)-7 d$.

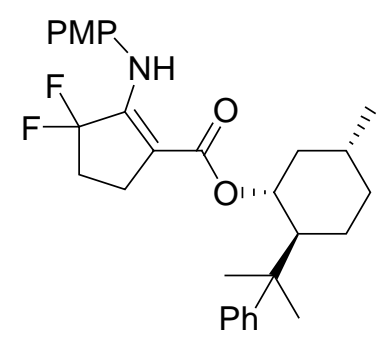

By means of General Procedure E, from $155 \mathrm{mg}(0.30 \mathrm{mmol})$ of $\mathbf{6 d}$, $120 \mathrm{mg}$ of $7 \mathbf{d}$ were obtained as a colorless oil (83\% yield). $R_{f}=0.35$ (hexane/EtOAc, $\left.5: 1\right) .[\alpha]^{25}{ }_{\mathrm{D}}=+54.0\left(c 0.9, \mathrm{CHCl}_{3}\right) .{ }^{1} \mathrm{H}$ NMR (300 MHz, CDCl $): \delta 0.81(\mathrm{~d}, J=6.4 \mathrm{~Hz}, 3 \mathrm{H}), 0.85-1.15(\mathrm{~m}, 3 \mathrm{H}), 1.16(\mathrm{~s}, 3 \mathrm{H}), 1.29(\mathrm{~s}$, $3 \mathrm{H}), 1.38-1.45(\mathrm{~m}, 1 \mathrm{H}), 1.56-1.66(\mathrm{~m}, 3 \mathrm{H}), 1.82(\mathrm{br} \mathrm{d}, J=10.9 \mathrm{~Hz}, 1 \mathrm{H}), 1.94-2.12(\mathrm{~m}, 4 \mathrm{H}), 3.72$ (s, 3H), $4.92(\mathrm{td}, J=10.5,4.5 \mathrm{~Hz}, 1 \mathrm{H}), 6.76(\mathrm{~d}, J=9.0 \mathrm{~Hz}, 2 \mathrm{H}), 7.01-7.22(\mathrm{~m}, 7 \mathrm{H}), 8.66(\mathrm{~s}, 1 \mathrm{H})$. ${ }^{13} \mathrm{C}$ NMR $\left(75.5 \mathrm{MHz}, \mathrm{CDCl}_{3}\right): \delta 21.8,22.0,25.3,26.7,27.8,31.4,33.2\left(\mathrm{t},{ }^{2} J_{\mathrm{CF}}=24.4 \mathrm{~Hz}\right), 34.5$, 39.8, 42.3, 50.5, 55.4, 73.4, $101.9\left(\mathrm{t},{ }^{3} J_{\mathrm{CF}}=7.5 \mathrm{~Hz}\right), 113.9,124.9,125.1\left(\mathrm{t},{ }^{4} J_{\mathrm{CF}}=2.7 \mathrm{~Hz}\right), 125.4$, $127.9,128.9\left(\mathrm{t},{ }^{1} J_{\mathrm{CF}}=249.0 \mathrm{~Hz}\right), 131.9,148.9\left(\mathrm{t},{ }^{2} J_{\mathrm{CF}}=23.3 \mathrm{~Hz}\right), 151.6,156.9,166.9 .{ }^{19} \mathrm{~F} \mathrm{NMR}$ (282.4 MHz, $\left.\mathrm{CDCl}_{3}\right): \delta-87.73(\mathrm{br}, 2 \mathrm{~F})$. HRMS (EI) calcd for $\mathrm{C}_{29} \mathrm{H}_{35} \mathrm{~F}_{2} \mathrm{NO}_{3}\left(\mathrm{M}^{+}\right)$: 483.2585; found: 483.2556 .

Synthesis of $\quad(+)-\left(2 S, 3 S, 1^{\prime} R, 2^{\prime} S, 5^{\prime} R\right)-1,1-d i f l u o r o-2-(4-m e t h o x y p h e n y l a m i n o)-3-\left(8^{\prime}-\right.$ phenylmenthyloxycarbonyl)cyclopentane, $(+)-8 \mathrm{~d}$.

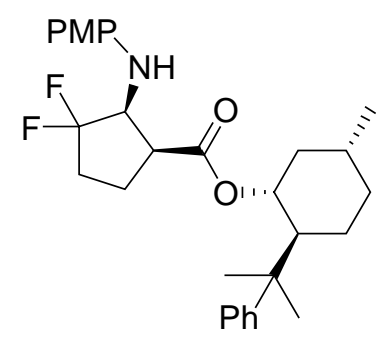


By means of General Procedure F, from $93 \mathrm{mg}(0.19 \mathrm{mmol})$ of $7 \mathbf{d}, 38 \mathrm{mg}$ of $\mathbf{8 d}$ were obtained as a colorless oil (41\% yield), together with $10 \mathrm{mg}$ of its diastereoisomer (11\% yield) and $31 \mathrm{mg}$ of recovered 7d (33\% yield). $R_{f}=0.15$ (hexane/EtOAc, $\left.15: 1\right) .[\alpha]^{25}{ }_{\mathrm{D}}=+27.1\left(\mathrm{c} 1.0, \mathrm{CHCl}_{3}\right) .{ }^{1} \mathrm{H}$ NMR (300 MHz, $\left.\mathrm{CDCl}_{3}\right): \delta 0.46(\mathrm{q}, J=11.8 \mathrm{~Hz}, 1 \mathrm{H}), 0.67(\mathrm{~d}, J=6.5 \mathrm{~Hz}, 3 \mathrm{H}), 0.68-0.84(\mathrm{~m}$, 2H), 0.94-1.10 (m, 2H), $1.11(\mathrm{~s}, 3 \mathrm{H}), 1.20(\mathrm{~s}, 3 \mathrm{H}), 1.23-1.35(\mathrm{~m}, 1 \mathrm{H}), 1.44-1.58(\mathrm{~m}, 2 \mathrm{H}), 1.60-$ $1.73(\mathrm{~m}, 2 \mathrm{H}), 1.87(\mathrm{td}, J=11.4,3.6 \mathrm{~Hz}, 1 \mathrm{H}), 1.93-2.12(\mathrm{~m}, 2 \mathrm{H}), 2.13-2.30(\mathrm{~m}, 1 \mathrm{H}), 3.61-3.71$ (m, 1H), $3.68(\mathrm{~s}, 3 \mathrm{H}), 4.68(\mathrm{td}, J=10.7,4.4 \mathrm{~Hz}, 1 \mathrm{H}), 6.44(\mathrm{~d}, J=8.9 \mathrm{~Hz}, 2 \mathrm{H}), 6.69$ (d, $J=8.9 \mathrm{~Hz}$, 2H), 7.04-7.14 (m, 1H), 7.16-7.25 (m, 4H). ${ }^{13} \mathrm{C}$ NMR (75.5 MHz, $\left.\mathrm{CDCl}_{3}\right): \delta$ 21.6, 21.9, 23.6, 26.2, 29.0, 31.1, 31.9 (t, $\left.{ }^{2} J_{\mathrm{CF}}=24.4 \mathrm{~Hz}\right), 34.4,39.4,41.1,44.3\left(\mathrm{~d},{ }^{3} J_{\mathrm{CF}}=3.5 \mathrm{~Hz}\right), 50.1,55.8,60.7$ $\left(\mathrm{dd},{ }^{2} J_{\mathrm{CF}}=30.3,20.6 \mathrm{~Hz}\right), 74.9,114.6,114.8,125.0,125.3,127.8,129.4\left(\mathrm{dd},{ }^{1} J_{\mathrm{CF}}=257.2,252.7\right.$ $\mathrm{Hz}), 140.8,151.9,152.6,171.2 .{ }^{19} \mathrm{~F}$ NMR $\left(282.4 \mathrm{MHz}, \mathrm{CDCl}_{3}\right) \delta-98.76\left(\mathrm{dtd}, J_{\mathrm{FF}}=228.9 \mathrm{~Hz}\right.$, $\left.J_{\mathrm{FH}}=16.1,9.1 \mathrm{~Hz}, 1 \mathrm{~F}\right),-109.20\left(\mathrm{dtd}, J_{\mathrm{FF}}=228.9 \mathrm{~Hz}, J_{\mathrm{FH}}=13.1,7.2 \mathrm{~Hz}, 1 \mathrm{~F}\right)$. HRMS (EI) calcd for $\mathrm{C}_{29} \mathrm{H}_{37} \mathrm{~F}_{2} \mathrm{NO}_{3}\left(\mathrm{M}^{+}\right)$: 485.2742 ; found: 485.2748 .

Data of the $(2 R, 3 R)$ diastereoisomer of $\mathbf{8 d}$ :

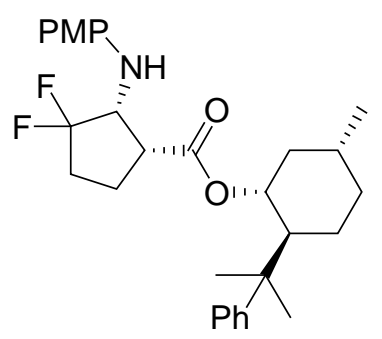

$R_{f}=0.18\left(\right.$ hexane/EtOAc, 15:1). $[\alpha]^{25}=-13.1\left(\right.$ c 1.3, $\left.\mathrm{CHCl}_{3}\right) .{ }^{1} \mathrm{H}$ NMR $\left(300 \mathrm{MHz}, \mathrm{CDCl}_{3}\right): \delta$ 0.77-0.82 (m, 1H), $0.82(\mathrm{~d}, J=6.5 \mathrm{~Hz}, 3 \mathrm{H}), 0.89$ (q, $J=11.6 \mathrm{~Hz}, 1 \mathrm{H}), 0.96-1.09$ (m, 2H), 1.05 (s, $3 \mathrm{H}), 1.12(\mathrm{~s}, 3 \mathrm{H}), 1.16-1.28(\mathrm{~m}, 1 \mathrm{H}), 1.29-1.49(\mathrm{~m}, 1 \mathrm{H}), 1.53-1.72(\mathrm{~m}, 4 \mathrm{H}), 1.80$ (br d, J= 12.1 $\mathrm{Hz}, 1 \mathrm{H}), 1.89-2.28(\mathrm{~m}, 3 \mathrm{H}), 2.49(\mathrm{q}, J=7.2 \mathrm{~Hz}, 1 \mathrm{H}), 3.68(\mathrm{~s}, 3 \mathrm{H}), 3.73-3.85(\mathrm{~m}, 1 \mathrm{H}), 4.74$ (td, 
$J=10.7,4.4 \mathrm{~Hz}, 1 \mathrm{H}), 6.64(\mathrm{~d}, J=9.0 \mathrm{~Hz}, 2 \mathrm{H}), 6.73(\mathrm{~d}, J=9.1 \mathrm{~Hz}, 2 \mathrm{H}), 7.00-7.06(\mathrm{~m}, 1 \mathrm{H}), 7.09-$ $7.20(\mathrm{~m}, 4 \mathrm{H}) .{ }^{13} \mathrm{C}$ NMR $\left(75.5 \mathrm{MHz}, \mathrm{CDCl}_{3}\right): \delta 21.8,22.7,24.4,26.5,28.0,31.3,32.3\left(\mathrm{t},{ }^{2} J_{\mathrm{CF}}=\right.$ $24.9 \mathrm{~Hz}), 34.5,39.5,41.5,43.9\left(\mathrm{~d},{ }^{3} J_{\mathrm{CF}}=5.5 \mathrm{~Hz}\right), 50.2,55.7,61.2\left(\mathrm{dd},{ }^{2} J_{\mathrm{CF}}=29.5,21.3 \mathrm{~Hz}\right)$, 75.4, 114.8, 115.5, 128.0, 125.3, 127.9, $129.1\left(\mathrm{t},{ }^{1} J_{\mathrm{CF}}=254.6 \mathrm{~Hz}\right), 140.7,151.6,152.9,171.6 .{ }^{19} \mathrm{~F}$ $\operatorname{NMR}\left(282.4 \mathrm{MHz}, \mathrm{CDCl}_{3}\right) \delta-96.74\left(\mathrm{dtd}, J_{\mathrm{FF}}=230.4 \mathrm{~Hz}, J_{\mathrm{FH}}=15.8,10.9 \mathrm{~Hz}, 1 \mathrm{~F}\right),-105.43$ (ddt, $\left.J_{\mathrm{FF}}=230.2 \mathrm{~Hz}, J_{\mathrm{FH}}=14.5,10.0 \mathrm{~Hz}, 1 \mathrm{~F}\right)$. HRMS (EI) calcd for $\mathrm{C}_{29} \mathrm{H}_{37} \mathrm{~F}_{2} \mathrm{NO}_{3}\left(\mathrm{M}^{+}\right): 485.2742$; found: 485.2735 .

\section{Synthesis of (+)-(2S,3S)-1,1-difluoro-3-(hydroxymethyl)-2-(4-methoxyphenylamino)-} cyclopentane, $(+)-13$.

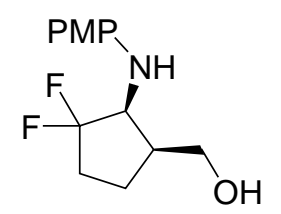

$\mathrm{LiAlH}_{4}(38 \mathrm{mg}, 1.00 \mathrm{mmol})$ was added to a cold $\left(0{ }^{\circ} \mathrm{C}\right)$ solution of $8 \mathbf{8 d}(97 \mathrm{mg}, 0.20 \mathrm{mmol})$ in THF ( $2 \mathrm{~mL})$. After stirring at room temperature for $6 \mathrm{~h}$, the reaction was quenched with $\mathrm{H}_{2} \mathrm{O}$ (10 $\mathrm{mL}$ ) and then potassium sodium tartrate $(50 \mathrm{mg})$ was added. The aqueous layer was extracted with EtOAc $(3 \times 10 \mathrm{~mL})$, dried over $\mathrm{Na}_{2} \mathrm{SO}_{4}$, and concentrated. The crude reaction mixture was purified by means of column chromatography on silica gel (hexane/EtOAc, 5:1 to 1:1) to obtain $41 \mathrm{mg}$ of $\mathbf{1 3}$ as a colorless oil (80 yield). $R_{f}=0.25$ (hexane/EtOAc, $\left.1: 1\right) \cdot[\alpha]^{25}{ }_{\mathrm{D}}=+7.9(c 0.5$, $\left.\mathrm{CHCl}_{3}\right) .{ }^{1} \mathrm{H} \mathrm{NMR}\left(300 \mathrm{MHz}, \mathrm{CDCl}_{3}\right): \delta 1.58-1.72(\mathrm{~m}, 1 \mathrm{H}), 1.82-1.96(\mathrm{~m}, 1 \mathrm{H}), 1.98-2.27(\mathrm{~m}$, 2H), 2.52-2.66 (m, 1H), 3.57 (dd, $J=11.3,5.0 \mathrm{~Hz}, 1 \mathrm{H}), 3.69$ (s, 3H), $3.70(\mathrm{dd}, J=11.2,7.6 \mathrm{~Hz}$, 1H), $3.89(\mathrm{dt}, J=14.0,9.6 \mathrm{~Hz}, 1 \mathrm{H}), 6.64(\mathrm{~d}, J=9.0 \mathrm{~Hz}, 2 \mathrm{H}), 6.74(\mathrm{~d}, J=9.1 \mathrm{~Hz}, 2 \mathrm{H}) .{ }^{13} \mathrm{C}$ NMR $\left(75.5 \mathrm{MHz}, \mathrm{CDCl}_{3}\right): \delta 22.1\left(\mathrm{dd},{ }^{3} J_{\mathrm{CF}}=5.5,3.1 \mathrm{~Hz}\right), 32.3\left(\mathrm{t},{ }^{2} J_{\mathrm{CF}}=23.8 \mathrm{~Hz}\right), 40.6\left(\mathrm{~d},{ }^{3} J_{\mathrm{CF}}=6.1\right.$ 
$\mathrm{Hz}), 55.8,62.1\left(\mathrm{dd},{ }^{2} J_{\mathrm{CF}}=25.3,19.2 \mathrm{~Hz}\right), 63.0\left(\mathrm{~d},{ }^{4} J_{\mathrm{CF}}=1.2 \mathrm{~Hz}\right), 115.0,115.6,129.8\left(\mathrm{dd},{ }^{2} J_{\mathrm{CF}}=\right.$ 254.4, 250.2 Hz), 141.3, 153.3. ${ }^{19} \mathrm{~F}$ NMR $\left(282.4 \mathrm{MHz}, \mathrm{CDCl}_{3}\right) \delta-100.79\left(\mathrm{dq}, J_{\mathrm{FF}}=229.4 \mathrm{~Hz}\right.$, $\left.J_{\mathrm{FH}}=10.4 \mathrm{~Hz}, 1 \mathrm{~F}\right),-107.89\left(\mathrm{dtd}, J_{\mathrm{FF}}=229.0 \mathrm{~Hz}, J_{\mathrm{FH}}=19.7,15.6 \mathrm{~Hz}, 1 \mathrm{~F}\right)$. HRMS (EI) calcd for $\mathrm{C}_{13} \mathrm{H}_{17} \mathrm{~F}_{2} \mathrm{NO}_{2}\left(\mathrm{M}^{+}\right): 257.1227$; found: 257.1165.

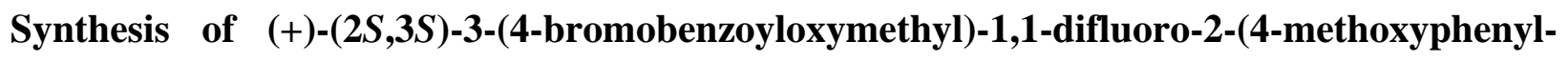
amino)cyclopentane, $(+)-14$.

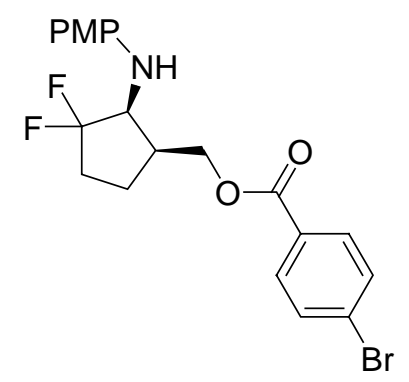

$\mathrm{Et}_{3} \mathrm{~N}(67 \mu \mathrm{L}, 0.48 \mathrm{mmol}), p$-bromobenzoyl chloride (105 $\left.\mathrm{mg}, 0.48 \mathrm{mmol}\right)$, and DMAP (10 mg, $0.08 \mathrm{mmol})$ were all added to a cold $\left(0^{\circ} \mathrm{C}\right)$ solution of $13(41 \mathrm{mg}, 0.16 \mathrm{mmol})$ in $\mathrm{CH}_{2} \mathrm{Cl}_{2}(1.6$ $\mathrm{mL})$. After stirring at room temperature for $3 \mathrm{~h}$, the reaction was quenched with $\mathrm{H}_{2} \mathrm{O}(10 \mathrm{~mL})$. The aqueous layer was extracted with $\mathrm{CH}_{2} \mathrm{Cl}_{2}(3 \times 10 \mathrm{~mL})$, dried over $\mathrm{Na}_{2} \mathrm{SO}_{4}$, and concentrated. The crude reaction mixture was purified by means of column chromatography on silica gel (hexane/EtOAc, 10:1 to $2: 1$ ) to obtain $61 \mathrm{mg}$ of $\mathbf{1 4}$ as a colorless oil (87 yield). $R_{f}=0.50$ (hexane/EtOAc, 2:1). $[\alpha]^{25}{ }_{\mathrm{D}}=+41.3\left(\right.$ c $\left.2.3, \mathrm{CHCl}_{3}\right) .{ }^{1} \mathrm{H}$ NMR $\left(300 \mathrm{MHz}, \mathrm{CDCl}_{3}\right): \delta 1.68-1.82$ (m, 1H), 1.95-2.32 (m, 3H), 2.81-2.94 (m, 1H), $3.66(\mathrm{~s}, 3 \mathrm{H}), 3.82$ (br, 1H), 4.00 (dt, J=14.9, 9.5 Hz, 1H), $4.22(\mathrm{dd}, J=11.4,6.4 \mathrm{~Hz}, 1 \mathrm{H}), 4.32(\mathrm{dd}, J=11.4,6.7 \mathrm{~Hz}, 1 \mathrm{H}), 6.54(\mathrm{~d}, J=8.8 \mathrm{~Hz}, 2 \mathrm{H})$, $6.67(\mathrm{~d}, J=9.0 \mathrm{~Hz}, 2 \mathrm{H}), 7.45(\mathrm{~d}, J=8.7 \mathrm{~Hz}, 2 \mathrm{H}), 7.66(\mathrm{~d}, J=8.6 \mathrm{~Hz}, 2 \mathrm{H}) .{ }^{13} \mathrm{C}$ NMR $(75.5 \mathrm{MHz}$, $\left.\mathrm{CDCl}_{3}\right): \delta 22.4,31.8\left(\mathrm{t},{ }^{2} J_{\mathrm{CF}}=24.0 \mathrm{~Hz}\right), 37.5\left(\mathrm{~d},{ }^{3} J_{\mathrm{CF}}=6.4 \mathrm{~Hz}\right), 55.6,60.2\left(\mathrm{dd},{ }^{2} J_{\mathrm{CF}}=25.0,19.7\right.$ $\mathrm{Hz}), 64.2,114.4,114.9,128.0,128.7,129.4\left(\mathrm{dd},{ }^{1} J_{\mathrm{CF}}=255.8,251.1 \mathrm{~Hz}\right), 131.0,131.6,140.7$, 
152.5, 165.7. ${ }^{19} \mathrm{~F}$ NMR $\left(282.4 \mathrm{MHz}, \mathrm{CDCl}_{3}\right) \delta-100.99\left(\mathrm{dq}, J_{\mathrm{FF}}=230.0 \mathrm{~Hz}, J_{\mathrm{FH}}=10.1 \mathrm{~Hz}, 1 \mathrm{~F}\right),-$ $107.46\left(\mathrm{dq}, J_{\mathrm{FF}}=229.6 \mathrm{~Hz}, J_{\mathrm{FH}}=17.4 \mathrm{~Hz}, 1 \mathrm{~F}\right)$. HRMS (EI) calcd for $\mathrm{C}_{20} \mathrm{H}_{20} \mathrm{BrF}_{2} \mathrm{NO}_{3}\left(\mathrm{M}^{+}\right)$: 439.0595; found: 439.0573 .

Synthesis of (+)-(2S,3S)-2-amino-3-(4-bromobenzoyloxymethyl)-1,1-difluorocyclopentane hydrochloride, (+)-15.

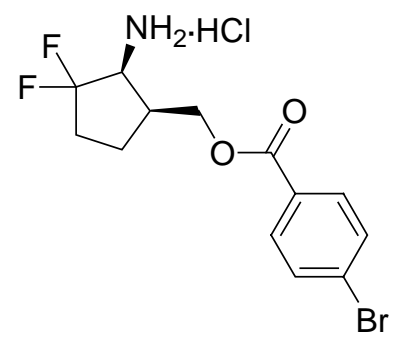

A solution of CAN (228 mg, $0.42 \mathrm{mmol})$ in $\mathrm{H}_{2} \mathrm{O}(1.5 \mathrm{~mL})$ was added dropwise to a cold $\left(0^{\circ} \mathrm{C}\right)$ solution of $14(61 \mathrm{mg}, 0.14 \mathrm{mmol})$ in $\mathrm{MeCN}(3.0 \mathrm{~mL})$. After stirring for $5 \mathrm{~h}$ at room temperature, the reaction was quenched with $5 \%$ aq. $\mathrm{NaHCO}_{3}$ until pH 7 , and $20 \%$ aq. $\mathrm{Na}_{2} \mathrm{~S}_{2} \mathrm{O}_{3}$ was added. The aqueous layer was extracted with EtOAc and the combined organic layers were dried over $\mathrm{Na}_{2} \mathrm{SO}_{4}$. The filtrate was concentrated and purified by means of flash chromatography on silica gel (hexane/EtOAc, 5:1 to 1:1) to obtain $39 \mathrm{mg}$ of the corresponding free amine as a colorless oil. This was dissolved in $\mathrm{Et}_{2} \mathrm{O}(1 \mathrm{~mL})$ and then anhydrous $\mathrm{HCl}\left(2 \mathrm{M}\right.$ in $\left.\mathrm{Et}_{2} \mathrm{O}, 1 \mathrm{~mL}\right)$ was added. The mixture was stirred at room temperature for $6 \mathrm{~h}$ and then concentrated. The residue was recrystallized $\left(\mathrm{MeOH} / \mathrm{i}-\mathrm{Pr}_{2} \mathrm{O}\right)$ to obtain $40 \mathrm{mg}$ of $\mathbf{1 5}$ as a white solid (78\% yield). Mp: $182-183{ }^{\circ} \mathrm{C} .[\alpha]^{25} \mathrm{D}^{2}=+1.1\left(c\right.$ 1.0, MeOH). ${ }^{1} \mathrm{H}$ NMR $\left(300 \mathrm{MHz}, \mathrm{CD}_{3} \mathrm{OD}\right): \delta 1.75-1.90(\mathrm{~m}$, 1H), 2.05-2.20 (m, 1H), 2.21-2.40 (m, 2H), $2.93(\mathrm{q}, J=6.9 \mathrm{~Hz}, 1 \mathrm{H}), 4.06(\mathrm{q}, J=9.8 \mathrm{~Hz}, 1 \mathrm{H})$, 4.30-4.42 (m, 2H), $7.62(\mathrm{~d}, J=8.3 \mathrm{~Hz}, 2 \mathrm{H}), 7.92(\mathrm{~d}, J=8.5 \mathrm{~Hz}, 2 \mathrm{H}) .{ }^{13} \mathrm{C}$ NMR $(75.5 \mathrm{MHz}$, $\left.\mathrm{CD}_{3} \mathrm{OD}\right): \delta 24.6,33.8\left(\mathrm{t},{ }^{2} J_{\mathrm{CF}}=23.6 \mathrm{~Hz}\right), 39.7\left(\mathrm{~d},{ }^{3} J_{\mathrm{CF}}=4.3 \mathrm{~Hz}\right), 58.5\left(\mathrm{dd},{ }^{2} J_{\mathrm{CF}}=30.4,20.2 \mathrm{~Hz}\right)$, 
65.0, 130.2, $130.7\left(\mathrm{t},{ }^{1} J_{\mathrm{CF}}=254.1 \mathrm{~Hz}\right), 130.9,133.3,133.9,167.8 .{ }^{19} \mathrm{~F}$ NMR $(282.4 \mathrm{MHz}$, $\left.\mathrm{CD}_{3} \mathrm{OD}\right) \delta-97.23\left(\mathrm{br} \mathrm{d}, J_{\mathrm{FF}}=237.1 \mathrm{~Hz}, 1 \mathrm{~F}\right),-102.93\left(\mathrm{dq}, J_{\mathrm{FF}}=237.1 \mathrm{~Hz}, J_{\mathrm{FH}}=15.8 \mathrm{~Hz}, 1 \mathrm{~F}\right)$. HRMS (FAB) calcd for $\mathrm{C}_{13} \mathrm{H}_{15} \mathrm{BrF}_{2} \mathrm{NO}_{2}\left(\mathrm{M}-\mathrm{Cl}^{+}\right)$: 334.0254 ; found: 334.0255 .

\section{X-ray data of (+)-15.}

The absolute structure of a single crystal of (+)-15 has been determined by X-ray diffraction studies. The structure shows two molecules in the asymmetric unit with an $(S)$ configuration for all the stereocenters (Figure 1). In the structure the cyclopentane ring adopts an envelope conformation with the 4-bromobenzoyloxymethyl group and the nitrogen atom showing a relative cis conformation. In the crystal all the ammonium hydrogen atoms form $\mathrm{N}-\mathrm{H}{ }^{\cdots} \mathrm{Cl}$ hydrogen bonds producing molecular ladders (Figure 2). C-H $\cdots$ F-C interactions are observed within ladders.

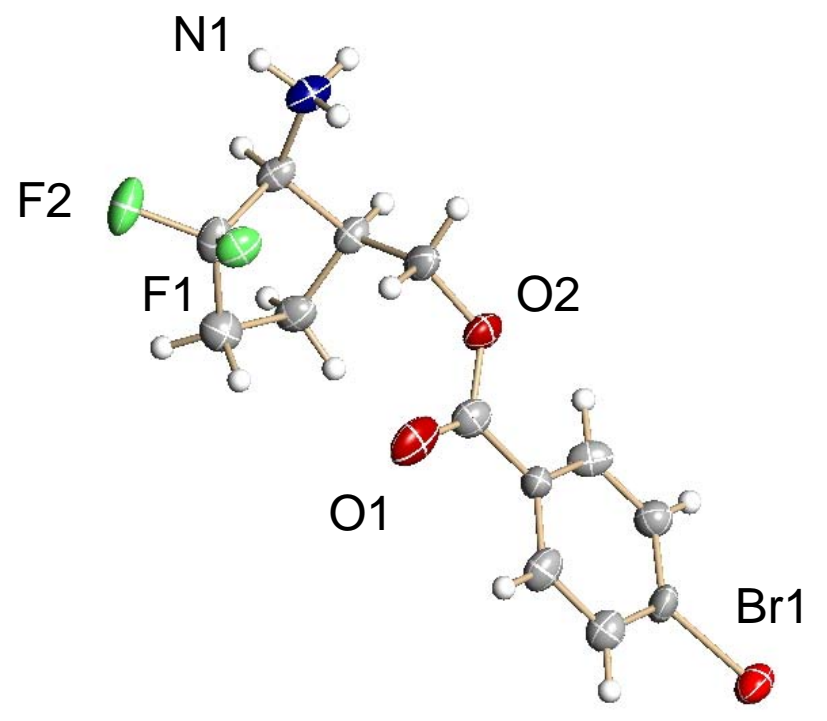

Figure 1. X-ray ellipsoid plot of compound (+)-15 (50\% probability level). 


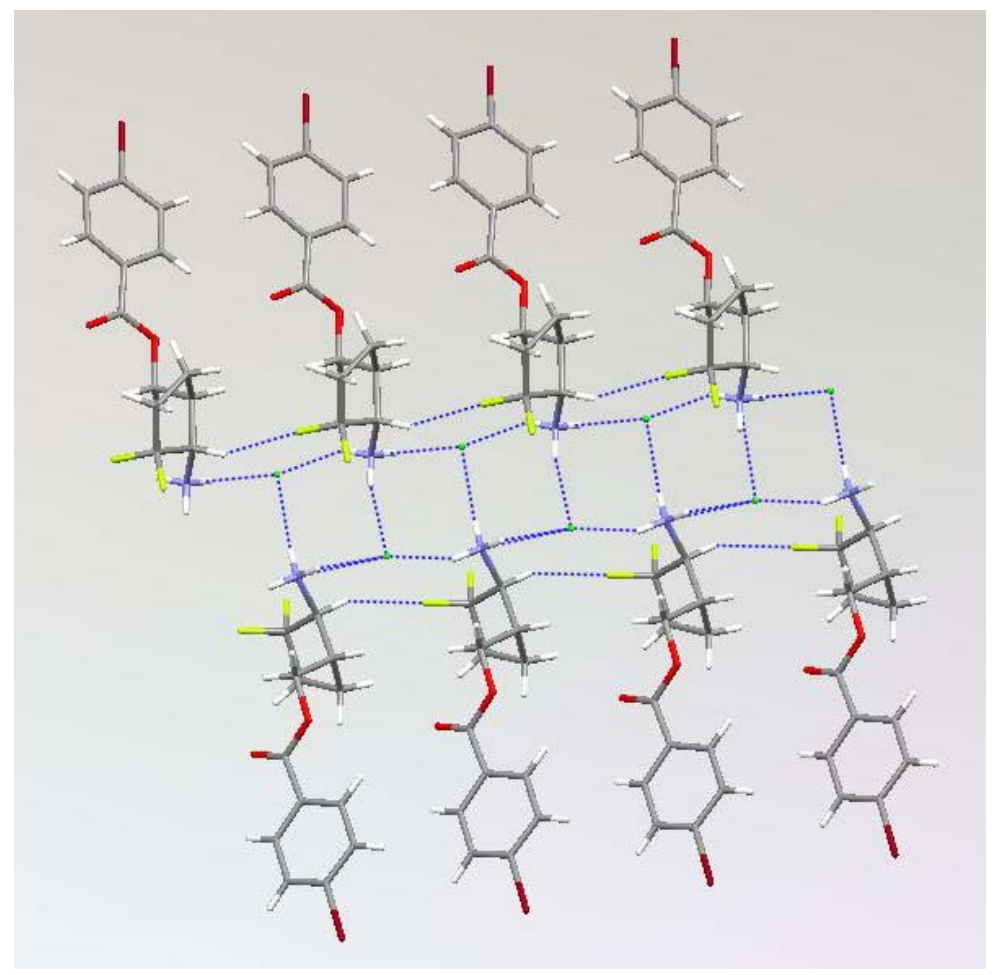

Figure 2. View of compound (+)-15 crystal packing showing the molecular ladder. Grey carbon, pale green fluorine, green chlorine, blue nitrogen and red oxygen atoms. $\mathrm{N}-\mathrm{H}^{\cdots}{ }^{\cdots} \mathrm{Cl}$ and $\mathrm{C}-\mathrm{H} \cdots \mathrm{F}-\mathrm{C}$ hydrogen bonds are shown in blue colour.

$X$-ray data for compound (+)-15: colourless lath, $0.52 \times 0.12 \times 0.02 \mathrm{~mm}$ size, monoclinic, $\mathrm{P} 2_{1}$, a $=15.334(3), \mathrm{b}=5.4149(11), \mathrm{c}=18.587(4) \AA, \beta=110.44(3) \AA, \mathrm{V}=1446.1(6) \AA^{3}, \mathrm{Z}=4, \rho_{\text {calcd }}=$ $1.702 \mathrm{gcm}^{-3}, \theta \max =30.00$, Mo K $\alpha, \lambda=0.71073 \AA, \omega$-scan, diffractometer Nonius Kappa $\mathrm{CCD}, \mathrm{T}=150(2) \mathrm{K}, 7307$ reflections collected of which 4760 were independent $\left(\mathrm{R}_{\mathrm{int}}=0.065\right)$, 3880 observed reflections $(\mathrm{I}>2 \sigma \mathrm{I})$, direct primary solution and refinement on $\mathrm{F}^{2}$ (SHELXS-97 and SHELXL-97, G.M. Sheldrick, University of Göttingen, 1997), 363 refined parameters, ammonium hydrogen atoms refined as rigid, others riding, the absolute structure was determined 
by anomalous dispersion effects (Flack parameter 0.06(19), H.D. Flack, Acta Cryst. A39, 876, 1983), $\mathrm{R}_{1}[\mathrm{I}>2 \sigma(\mathrm{I})]=0.0640, \mathrm{wR}_{2}$ (all data) $=0.1701$.

Table 1: Intermolecular hydrogen bonds $\mathrm{X}-\mathrm{H}^{\cdots} \mathrm{Y}$ lenthgs $(\AA)$ and angles $\left(^{\circ}\right)$ involved in the infinite ladder formation for compound (+)-15.

\begin{tabular}{|c|c|c|c|c|c|c|}
\hline H-bond & $\mathrm{X}-\mathrm{H}$ & $\mathrm{Y}$ & $X \cdot \cdot Y$ & $\mathrm{H} \cdot \mathrm{Y}$ & $\mathrm{XH} \cdot \cdot \mathrm{Y}$ & symmtery operator \\
\hline $\mathrm{N}-\mathrm{H} \cdots \mathrm{Cl}$ & N1-H1A & Cl1A & $3.142(7)$ & 2.30 & 154.6 & $\mathrm{x}, \mathrm{y}, \mathrm{z}$ \\
\hline $\mathrm{N}-\mathrm{H} \cdots \mathrm{Cl}$ & N1-H1B & $\mathrm{Cl} 1^{\mathrm{i}}$ & $3.123(8)$ & 2.26 & 157.7 & $-\mathrm{x}+1, \mathrm{y}+1 / 2,-\mathrm{z}+1$ \\
\hline $\mathrm{N}-\mathrm{H} \cdots \mathrm{Cl}$ & N1-H1C & $\mathrm{Cl} 1^{\mathrm{ii}}$ & $3.198(9)$ & 2.33 & 158.6 & $-x+1,-y-1 / 2,-z+1$ \\
\hline $\mathrm{N}-\mathrm{H} \cdots \mathrm{Cl}$ & N1A-H1D & $\mathrm{Cl} 1^{\mathrm{iii}}$ & $3.108(7)$ & 2.718 & 156.0 & $\mathrm{x}+1, \mathrm{y}, \mathrm{z}$ \\
\hline $\mathrm{N}-\mathrm{H} \cdots \mathrm{Cl}$ & N1A-H1E & $\mathrm{Cl}_{1} \mathrm{~A}^{\mathrm{iv}}$ & $3.116(8)$ & 2.22 & 166.2 & $-x, y+1 / 2,-z+1$ \\
\hline $\mathrm{N}-\mathrm{H} \cdots \mathrm{Cl}$ & N1A-H1F & $\mathrm{Cl}^{1} \mathrm{~A}^{\mathrm{v}}$ & $3.193(8)$ & 2.36 & 152.5 & $-\mathrm{x}, \mathrm{y}-1 / 2,-\mathrm{z}+1$ \\
\hline $\mathrm{C}-\mathrm{H} \cdots \mathrm{F}$ & C16-H16 & $\mathrm{F} 2^{\mathrm{vi}}$ & $3.237(11)$ & 2.35 & 147.3 & $x, y+2, z$ \\
\hline $\mathrm{C}-\mathrm{H} \cdots \mathrm{F}$ & C16A-H16A & $\mathrm{F} 2 \mathrm{~A}^{\mathrm{vi}}$ & $3.138(10)$ & 2.28 & 143.0 & $\mathrm{x}, \mathrm{y}+2, \mathrm{z}$ \\
\hline
\end{tabular}


SLLO $Z$

9LO'Z

60EI $2-$

L6EI' $\bar{C}$

L6ti'z-

ztol $z=$

$668 \mathrm{I} z$

osoz'

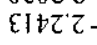

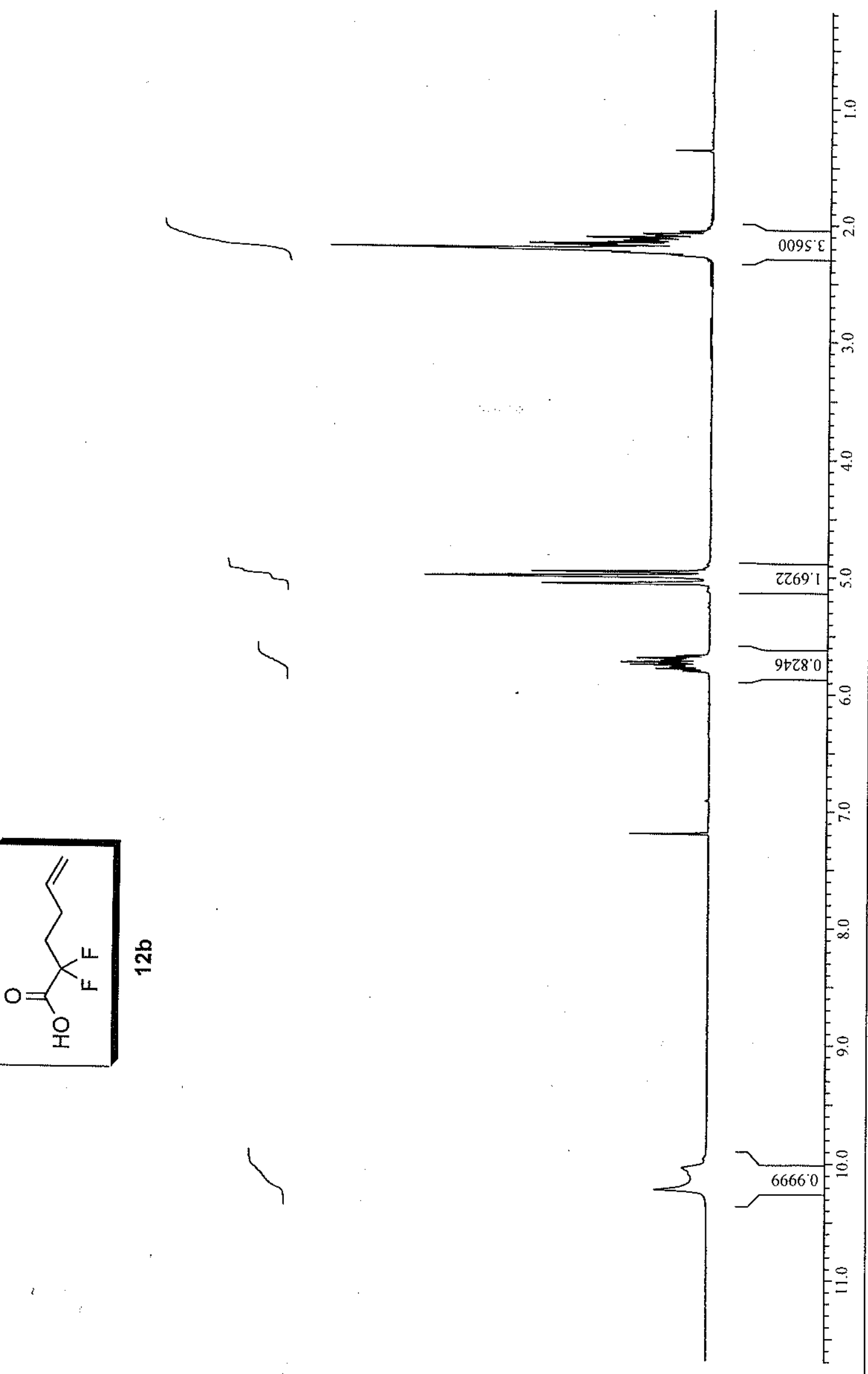

t $096^{\circ}$.

$\forall \angle 66 \circ-\sqrt{\Gamma}$

$0 \angle \$ 0 \subseteq-$

$\subseteq \varepsilon 699^{\circ}$

$t S 0 L S-7$

$6 \mathrm{ZI} L S$ '

$08 Z L S$

$\varepsilon 6+L \cdot S$

$10 L L S$

$6 E 8 L S$

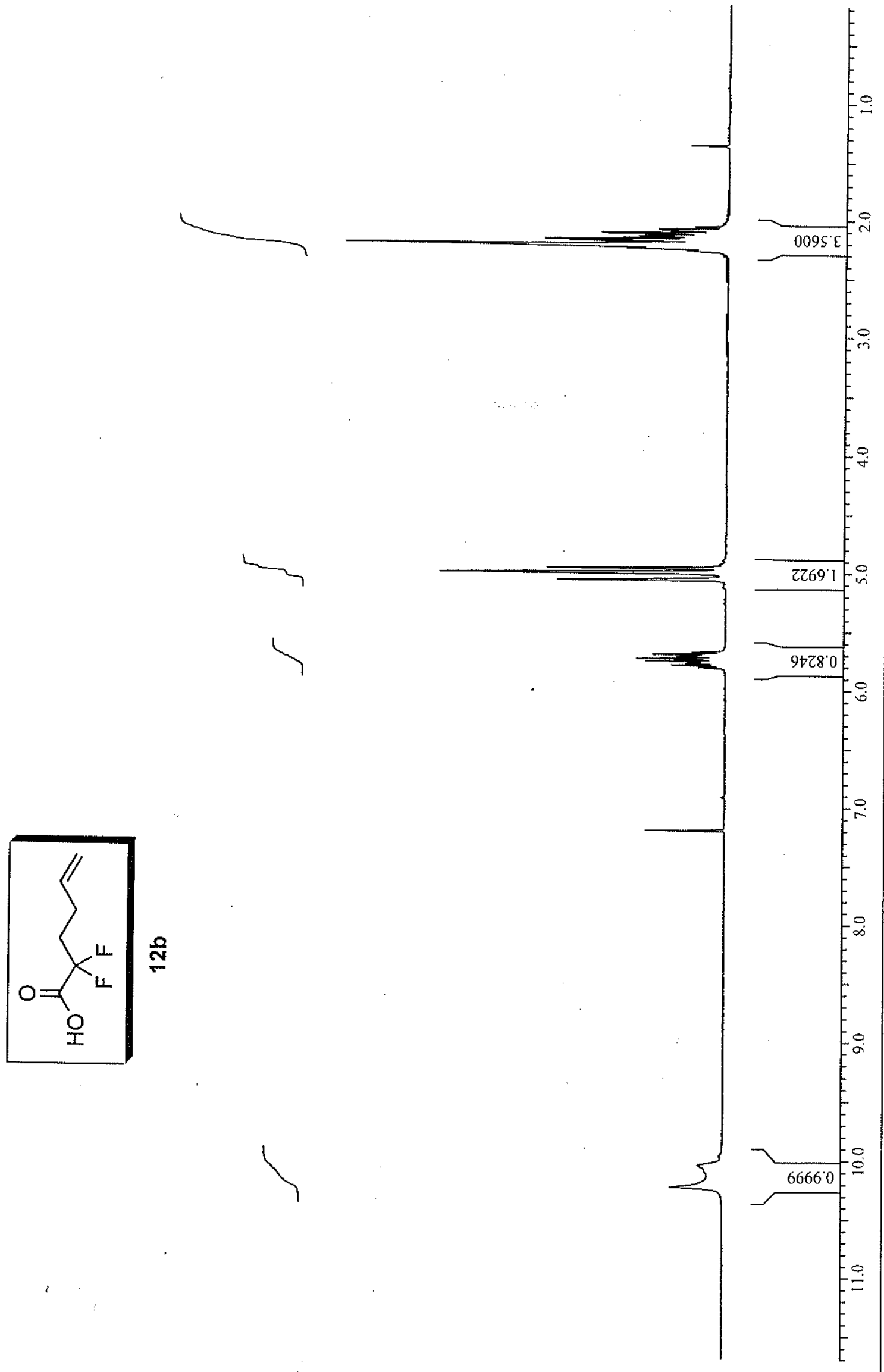

$681201-$ 


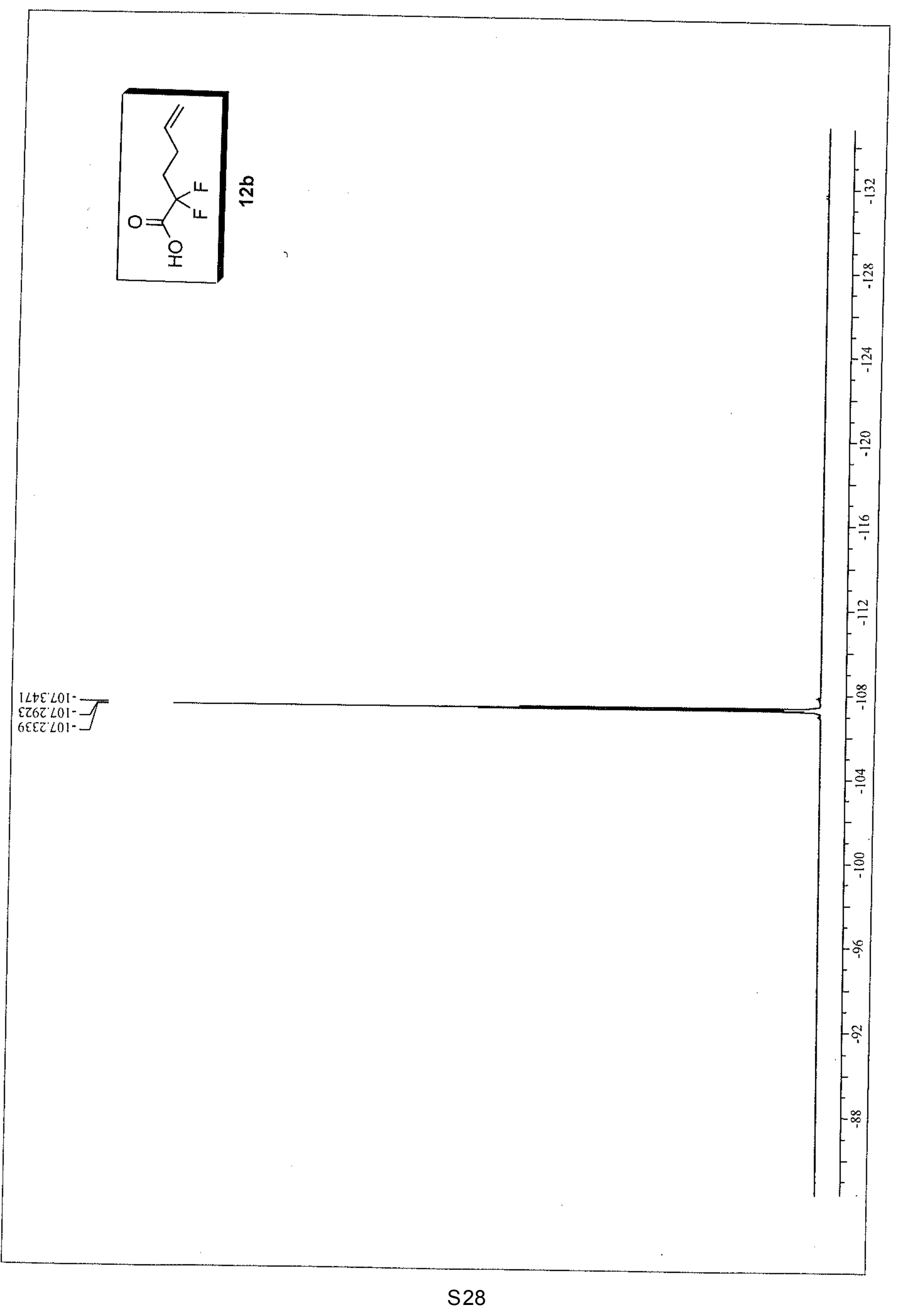




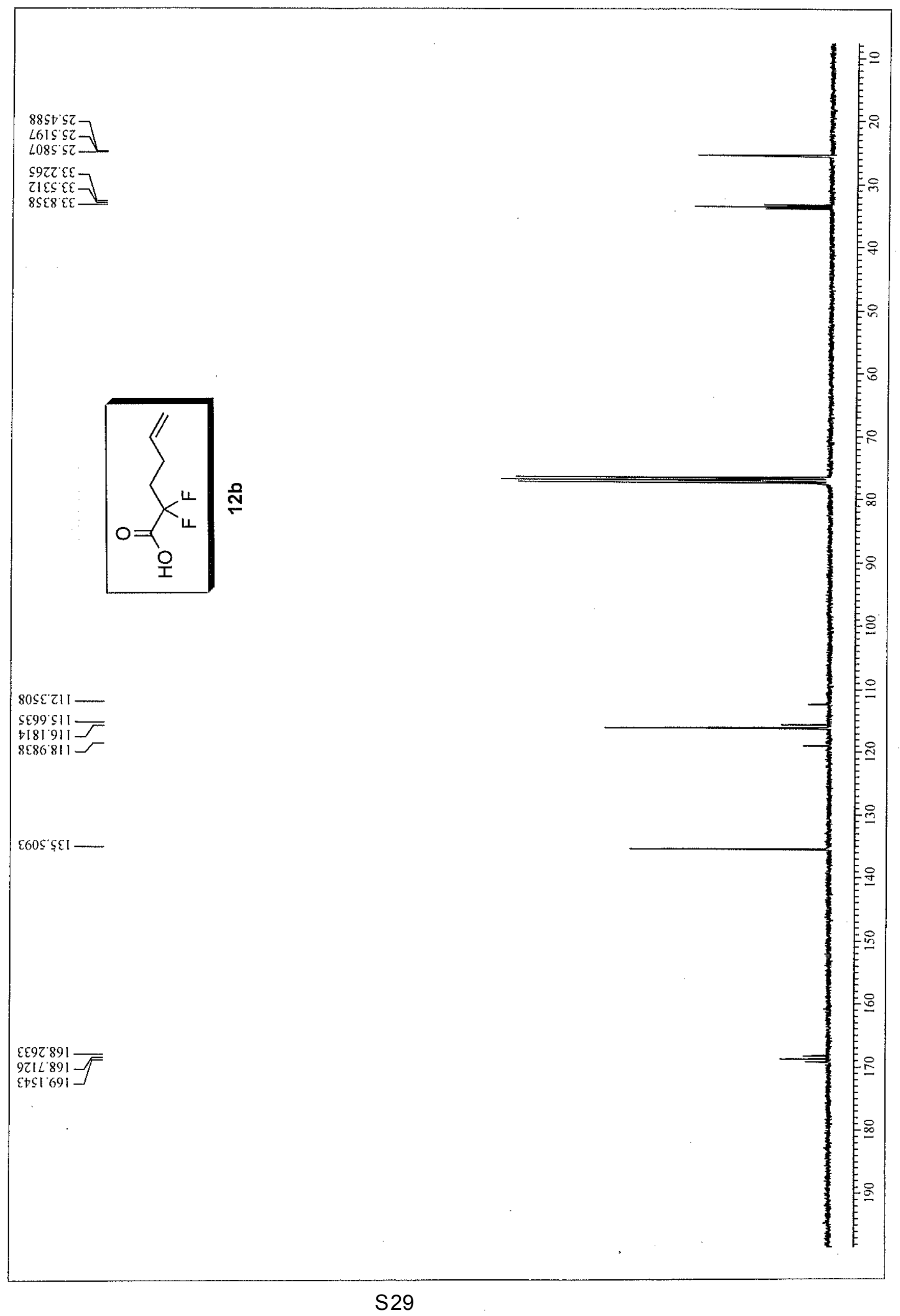


1605' I

ธEES I

LISS'I -

98\$s $1-$

$t z L S 1-$

6985 I -

0 वा9

2110 $z$

$\$ 620^{\circ} z-$

乙8£0 $Z$

$0<50 Z$ Z

t $190^{\circ} z$

$8590^{\circ} \mathrm{Z}$

$0 \$ 807$

ZLOI'

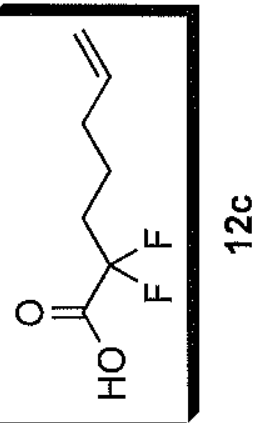

$2686^{\circ} t$

$t z+66^{\circ} \circ$

$\forall<t 6 \circ$

$0 \varepsilon 6^{\circ} \mathrm{t}-$

$\angle 8 \subseteq 6{ }^{\circ}-$

$2996^{\circ} \mathrm{t}$

$90 \angle 6 \circ-$

$1 \varepsilon \angle 6 \square-$

$69 L 66^{\circ}-$

I $500 \mathrm{~S}-$

$1010 \mathrm{~s}-$

$8 \subseteq 10 \varsigma$

ESEO S-

$\varepsilon \angle \varsigma 9^{\circ} \subseteq-$

$2699 \subseteq-$

$26 \angle 99^{\circ}-$

$\operatorname{tr} 69^{\circ} \varsigma-$

$8 \& I L ' S$

$\varepsilon 9 Z L \cdot S$

$+9 E L S-$

$\varepsilon 8 \sqcup L ' S-$

$20 L L^{\circ} \mathrm{S}$

$\{9956$

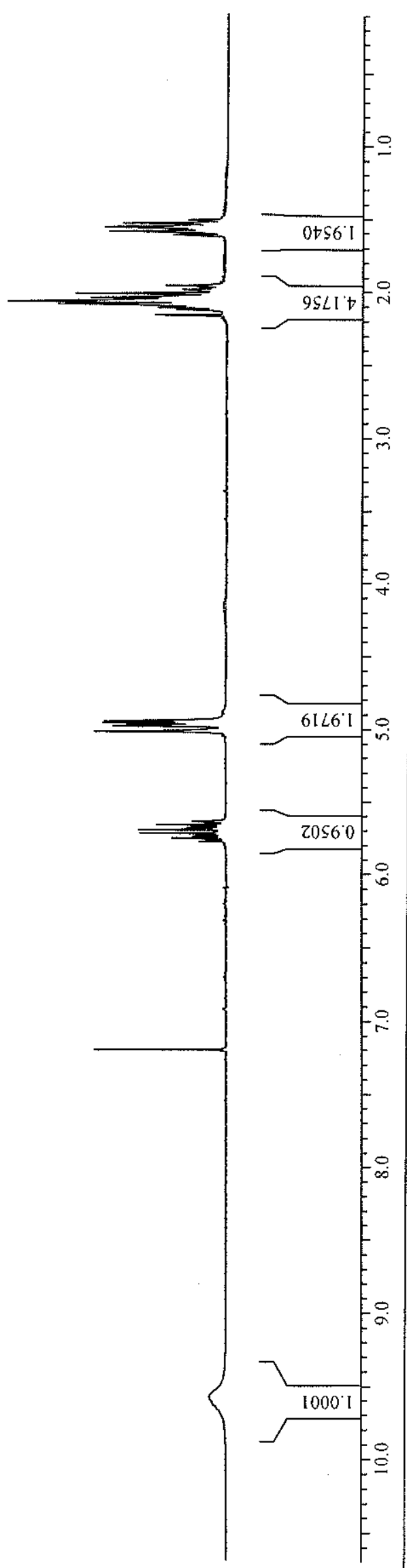


$\angle E 61^{\circ} \angle O \mathrm{I}^{-}-$

$\angle\left[E I^{\circ} \angle 01^{-}-\right.$
EELO $\angle 01^{-}$

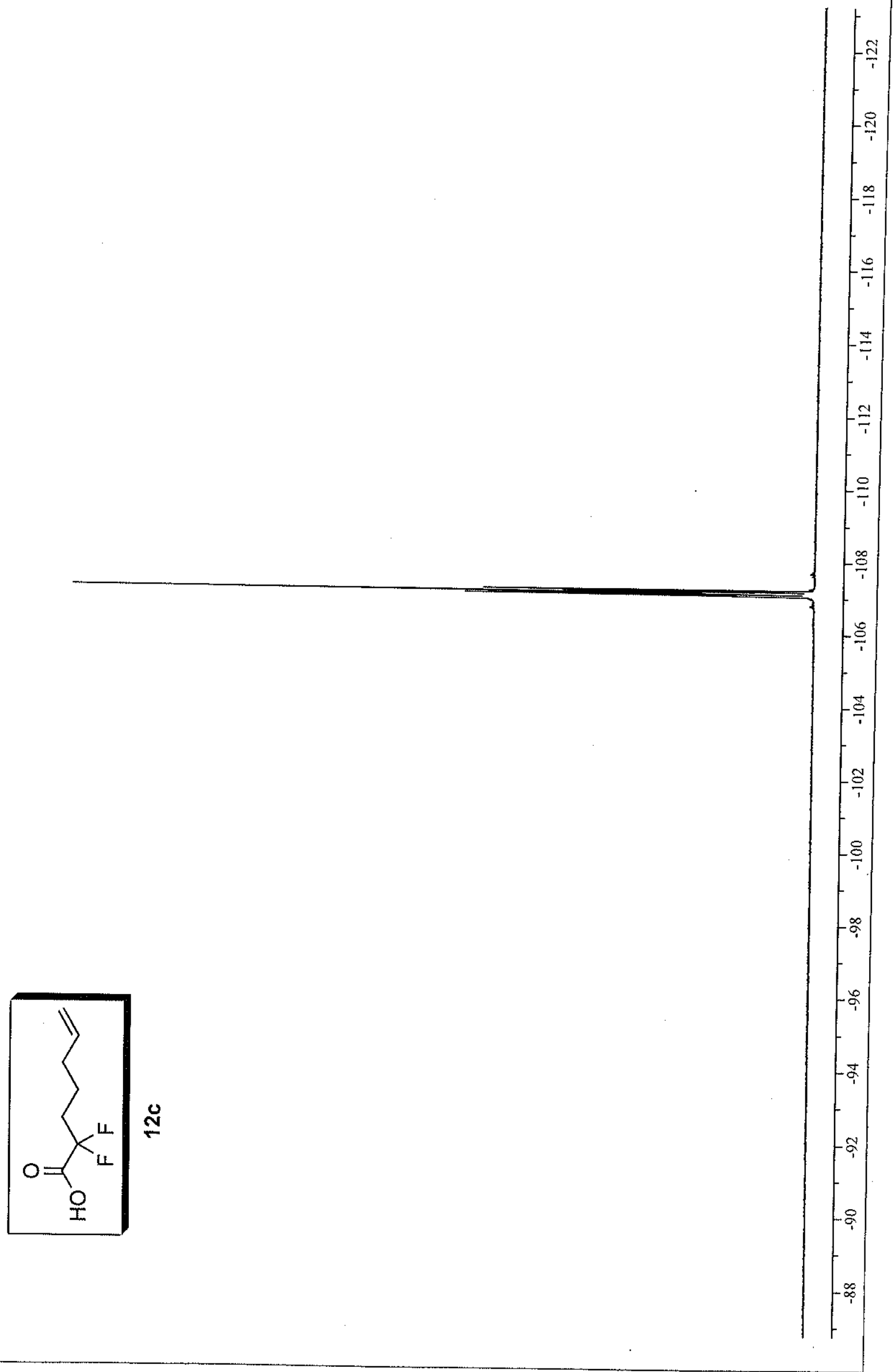




\section{$6 z 28 z \varepsilon$}

$9+9 z \varepsilon \varepsilon$

2695 $\varepsilon \varepsilon$

$6 \llbracket \angle 8 £ \varepsilon-$

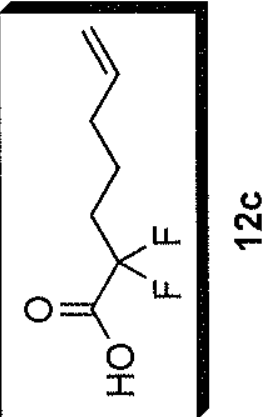

$\$ 198^{\circ} \subseteq 11-$

$18 \angle 0^{\circ} \angle E 1-$

1088891

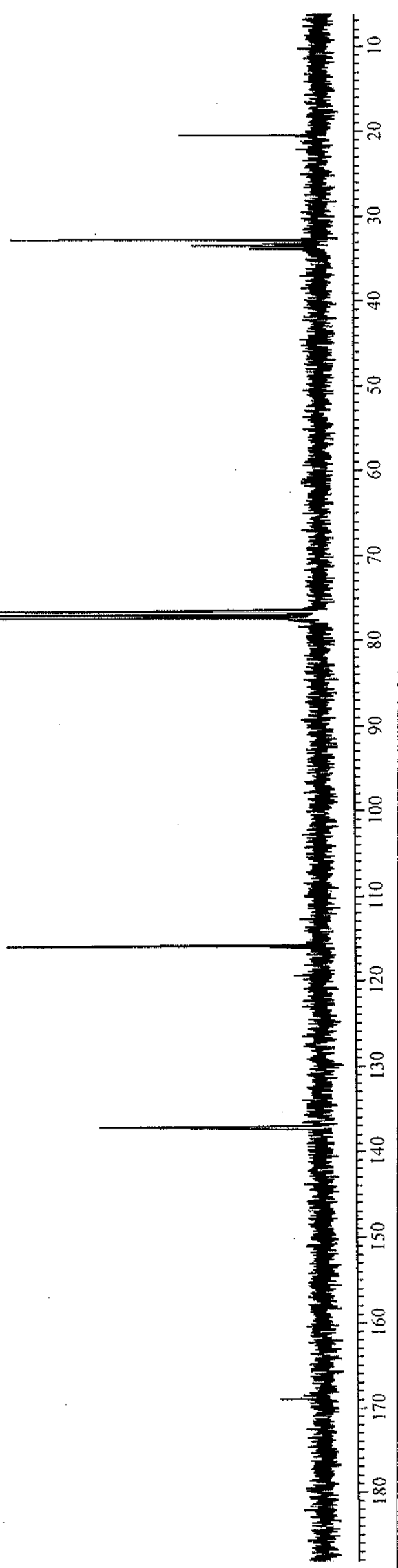




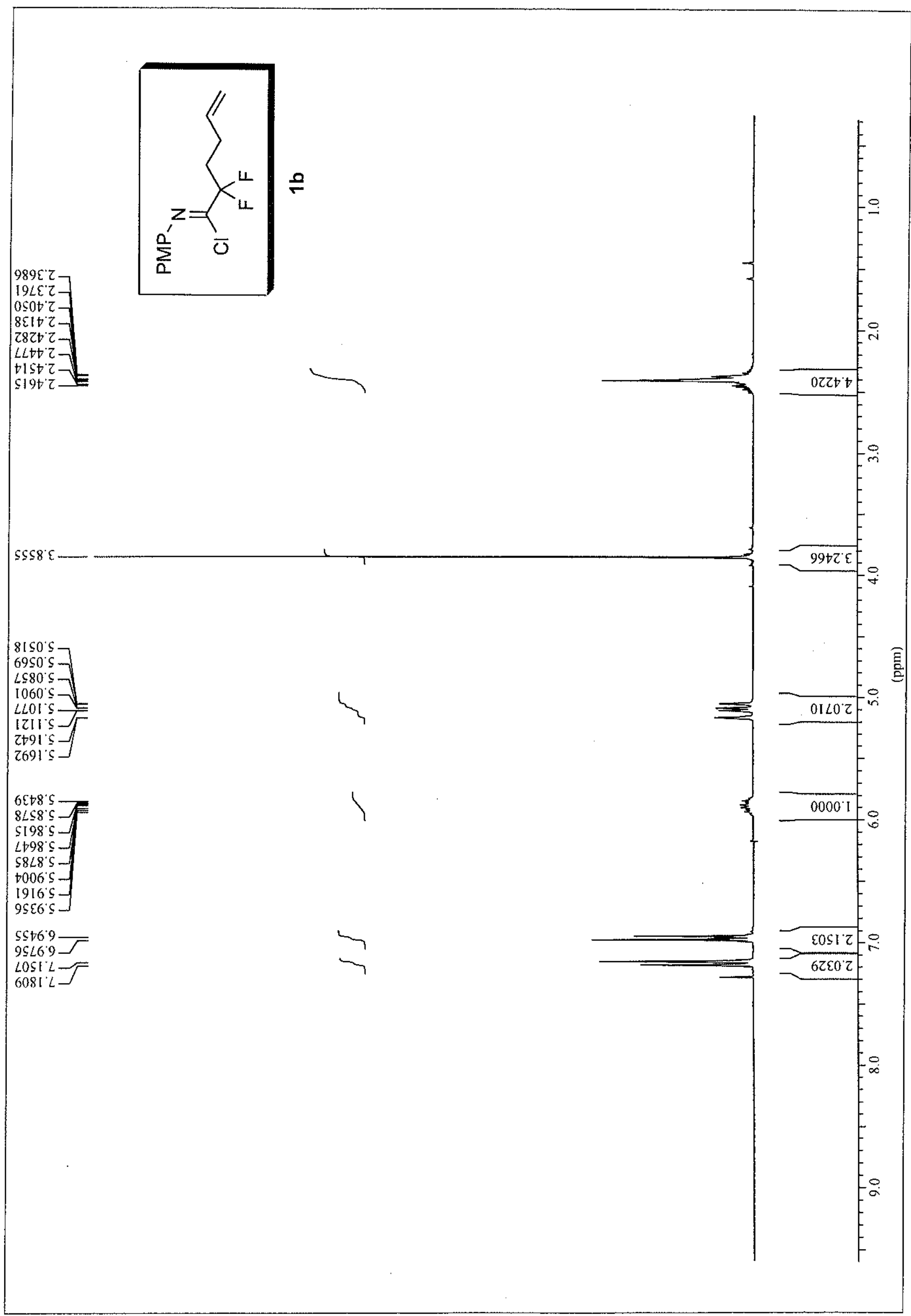




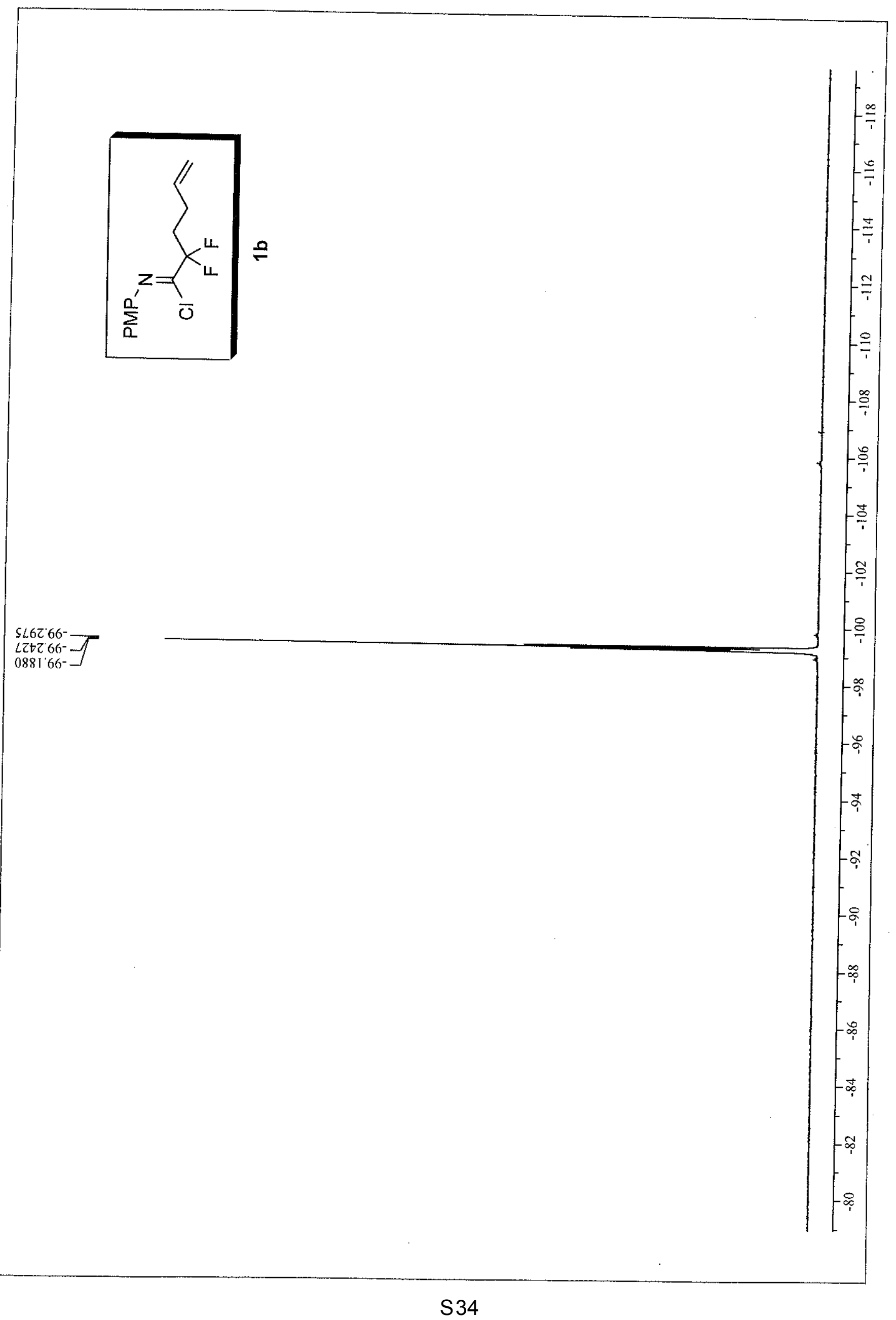


ILZ6 $\varepsilon \varepsilon$

$\downarrow 6 \varepsilon \mathcal{\circ} \downarrow \varepsilon$

$9[\operatorname{sct} t \varepsilon$

$\angle 0+T S \subseteq$

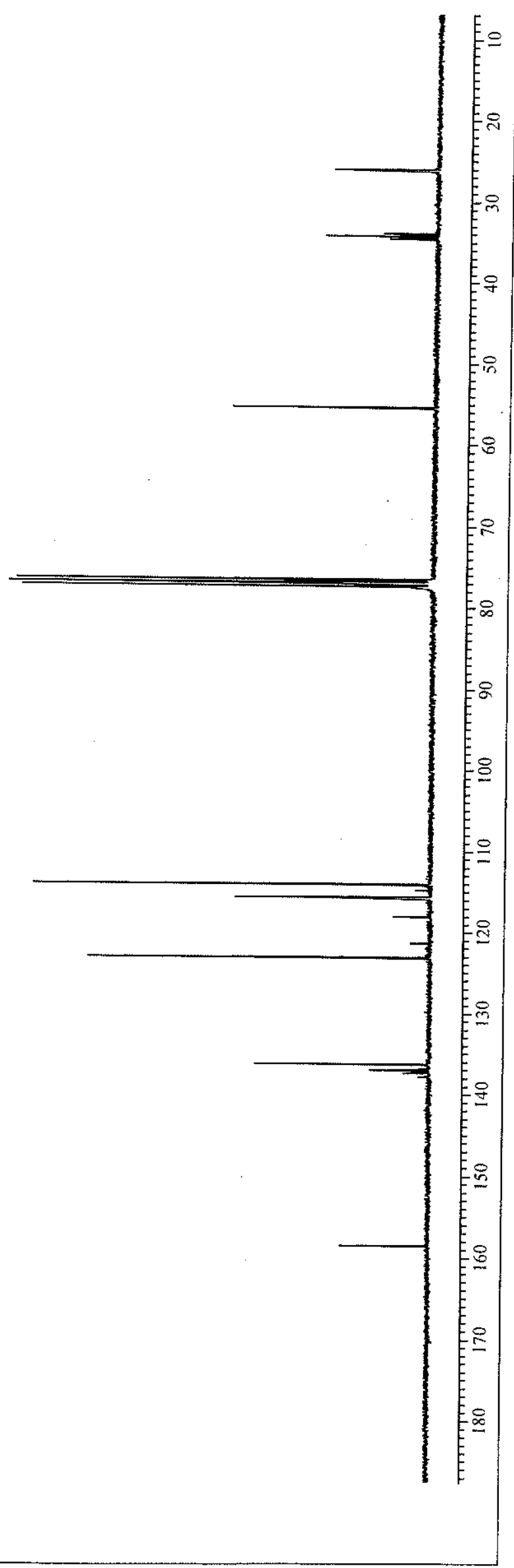

$2201+11$

6t9L'tl

to ZL SII

6IE0.811

6862 IZI -

Z†EIEZI

$198 Z^{\prime} 9 \varepsilon$

$6808^{\circ} 9 \mathrm{EL}$

Eจ66 9 \& I

$\varepsilon 16 Z^{\circ} L E I-$
$\angle 8 L L^{\circ} L E I$

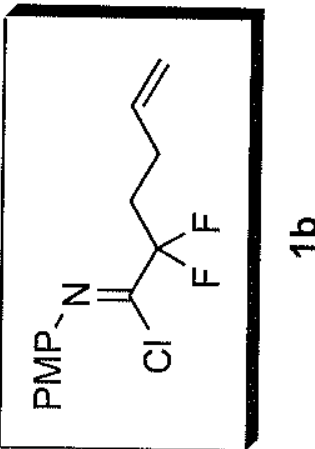

$\stackrel{2}{2}$

$\angle 0 £ \subseteq 8 S I-$ 


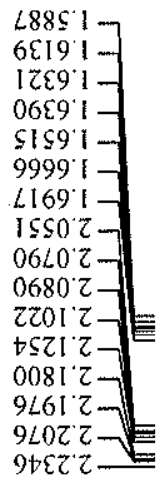

0689 1

SIS 1 -

$9999^{\circ} 1$

21691

$06 \angle 0$ Z

$0680^{\circ} \mathrm{Z}$

$2201^{\prime} z-$

tsZi'z]

$9 L 61^{\prime} z$

9L0てて

$9+\varepsilon \tau 2$
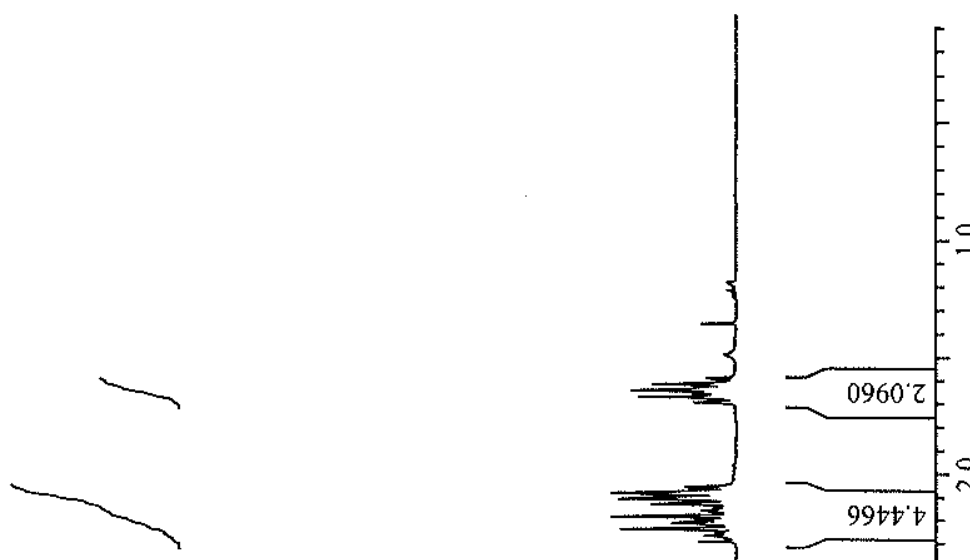

$0 \mid \subseteq L \mathcal{E}$

$00 S 0^{\circ} \varepsilon$

It $266^{\circ}$

$\therefore \angle 26^{\circ} \mathrm{O}$

จ0E6

$\varepsilon \nabla \subseteq 6^{\circ} t-$

$6656{ }^{\circ} \mathrm{t}$

$6 \pm 96 \circ-$

$0 L 10 \subseteq-$

$\angle Z Z O^{\circ} \mathrm{S}$

$8+99^{\circ} \varsigma-$

$8989^{\circ} \varsigma$

$\angle 869^{\circ} \mathrm{S}$

$\mathfrak{5} 60 L ' S$

EIZL'S

$6 \varepsilon+L S$

$8 \subseteq S L S$

$\$ 99 L^{\circ} \mathcal{S}$

$8 L L L ' S-$

t008. $\subseteq]$

$91+8 .=$

I $1288^{\circ}$

$1880 \%$

$2890 \mathrm{~L}-$

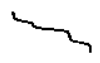

$2890^{\circ} \mathrm{C}$
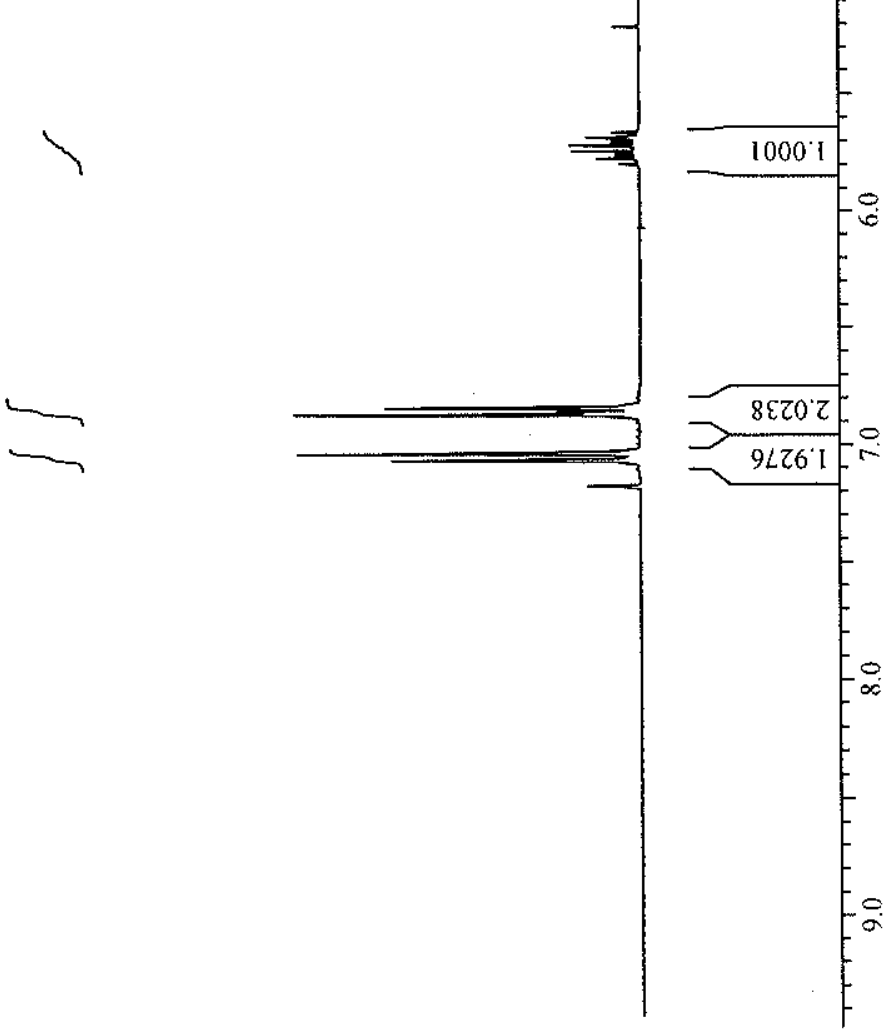


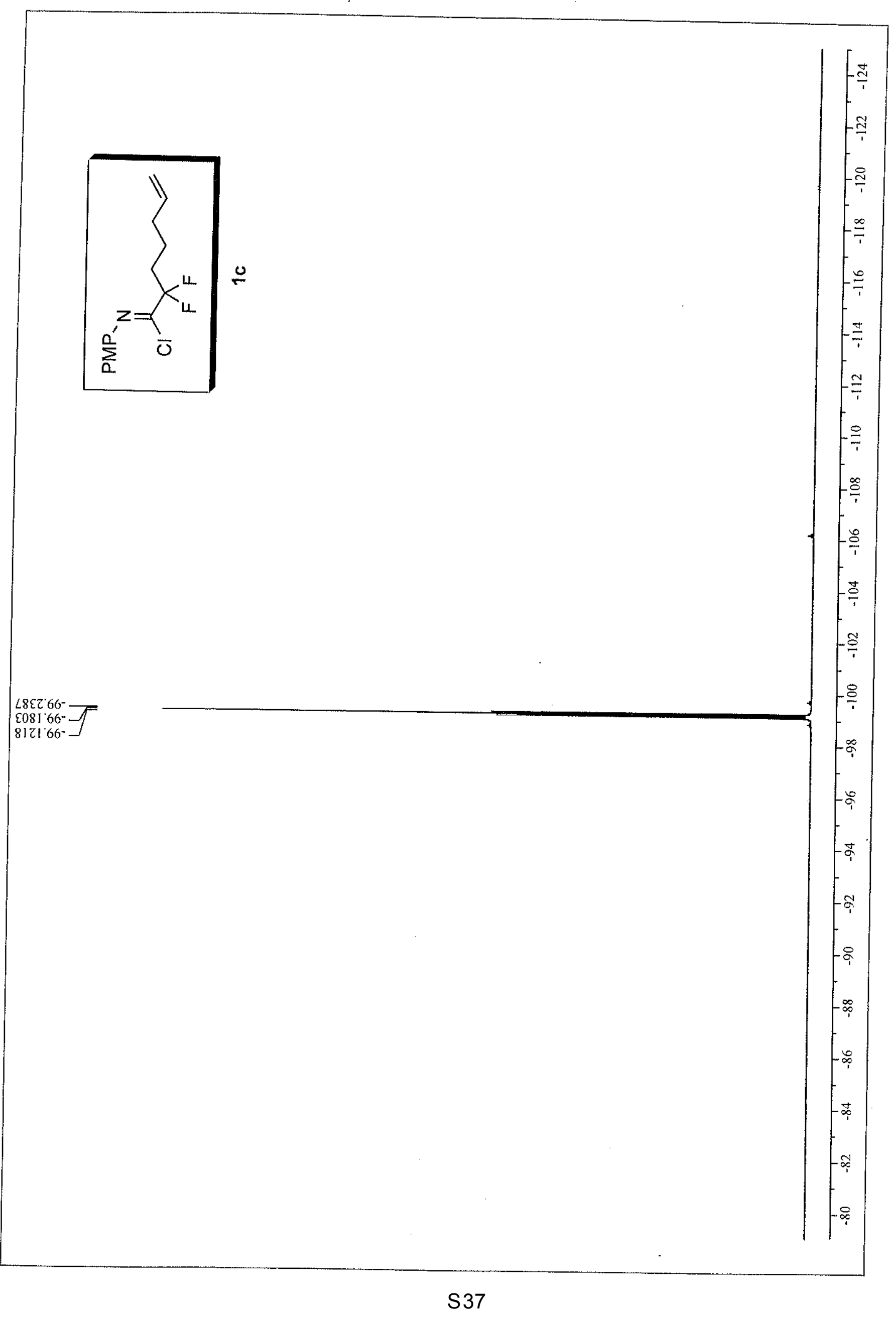



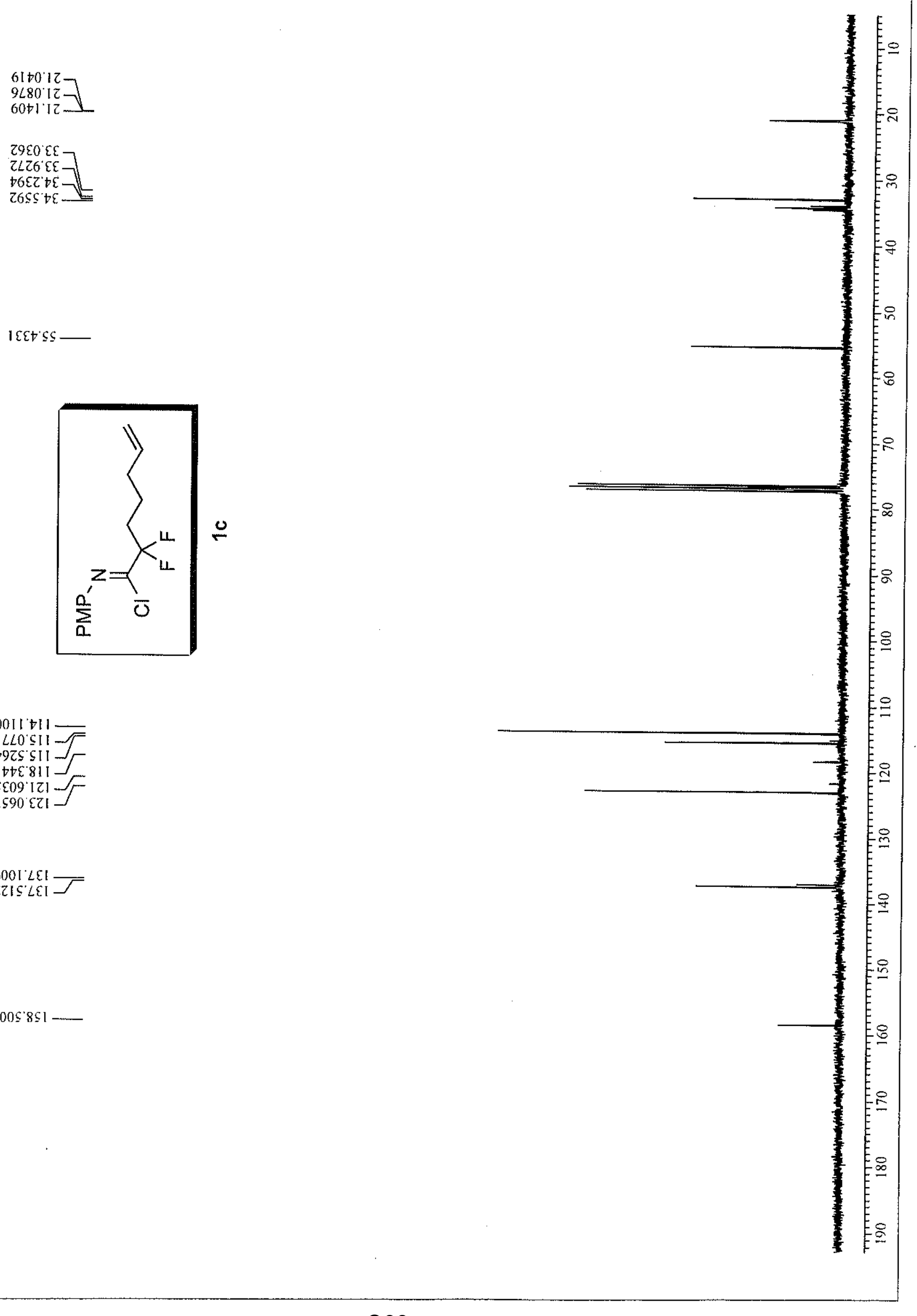


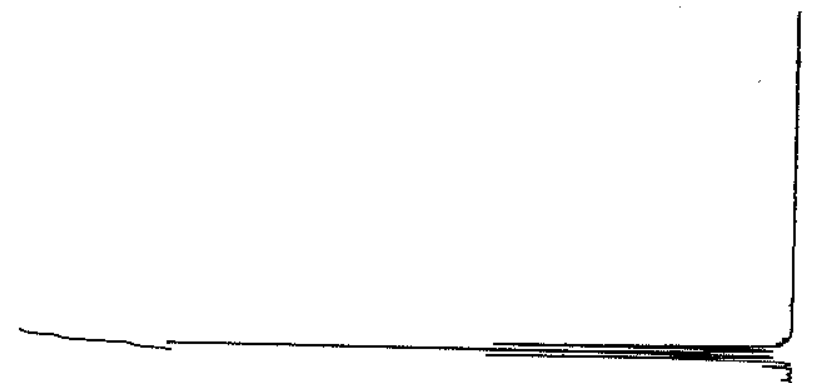

$\neg S \angle 0^{\circ} \varepsilon$

$5080^{\circ} \varepsilon-$

$6660^{\circ} \varepsilon$

$\varepsilon+0 I^{\circ} \varepsilon-$

र्टा ह

$0 Z \subseteq$ I $\mathcal{L}$

t9s $\varepsilon$

$9621 \mathcal{L}-$

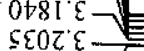

ธ80Z $\varsigma$

$9+9 L^{\prime}$

860 I' $t$

LEEI't
SLSI $=-1=$

$\angle 08 I^{\circ}-$

80965

$62109=$

1 2589

27889 -

$91069-=$

$90566^{\circ}-$

$\varepsilon 680^{\circ} \mathrm{L}-$

$+6 ! 1 \%$
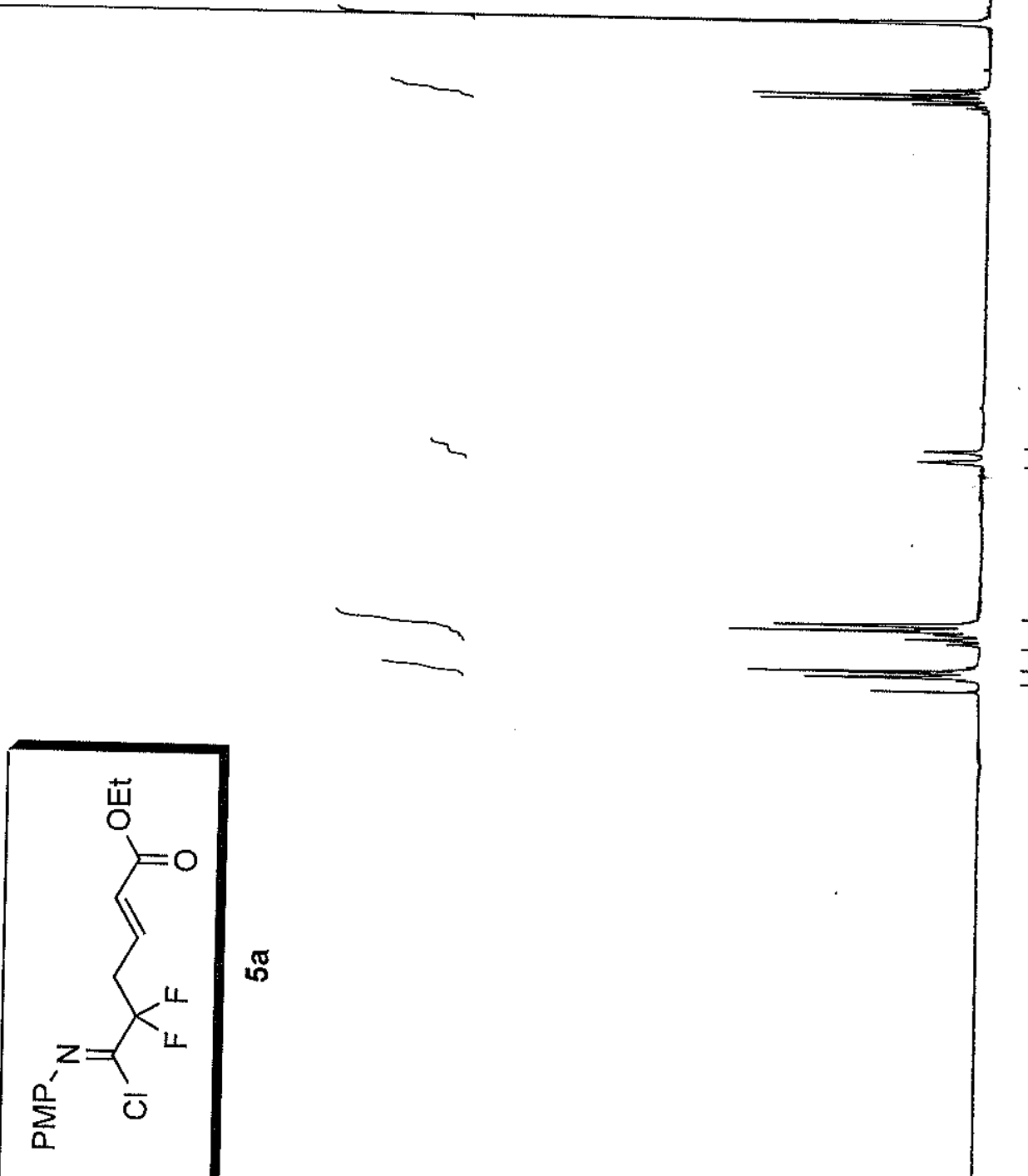

$8508^{\circ} \varepsilon$

z96z' $z$

in

is

$\stackrel{\circ}{+}$

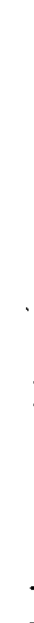




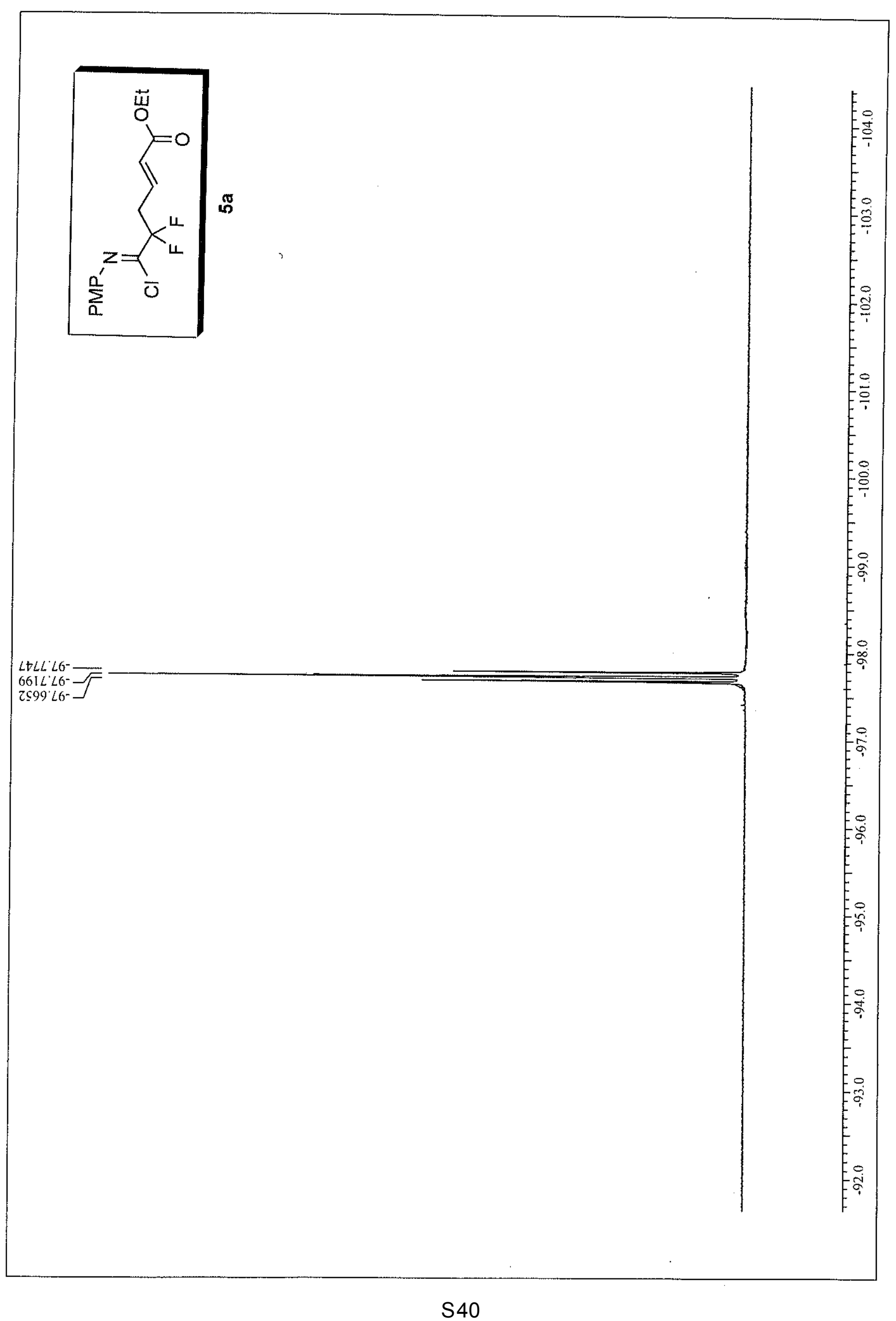




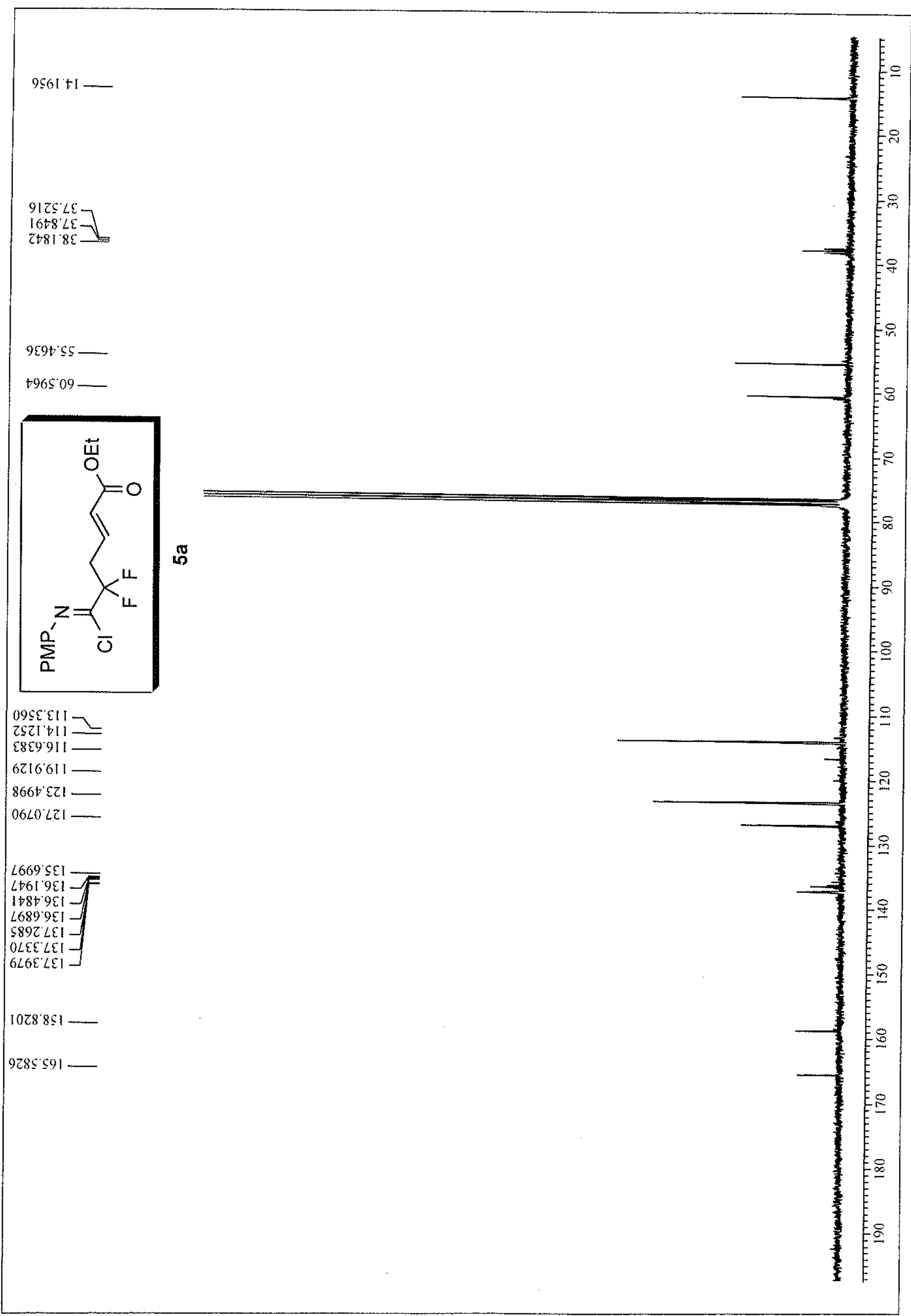


98LI'I

†ZOZ I

ع922.1]

$\checkmark \varepsilon Z C^{\prime}=$

S8Z\&' 2 -

I $6 \varepsilon \varepsilon \bar{\varepsilon}$

¿Zse

66ฐ\& $Z$

SSLE'

I $\varepsilon 8 \mathcal{E}^{\prime} \mathrm{Z}$

SZ6E'Z

6900' 2

68Zっ Z

\&E栱 2

ZLLV"

$\angle \mathcal{S t} \angle \mathcal{E}$

$\nabla \varepsilon \angle 0^{\circ} \mathrm{D}$

ZL60

l[ $6 \pm 1 \%$

$696 L^{\circ} \mathrm{S}$

$06 \pm 8^{\circ} \mathrm{C}-$

4
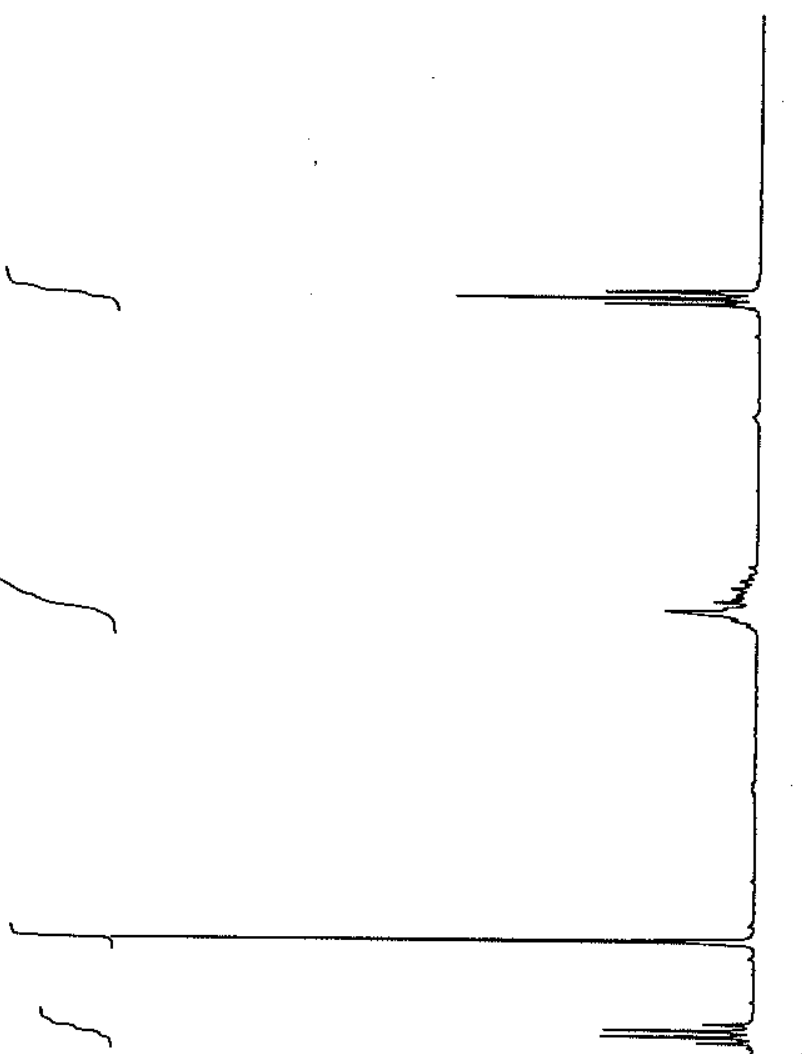

E

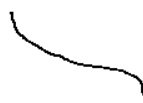

0L6L'

$5688^{\circ} 9$

$0698^{\circ} 9$

E216.

Z๖\&6.9-

$\varepsilon \forall \subseteq 6^{\circ}$

$S \varepsilon 90^{\circ} \mathrm{L}-$

$\angle \varepsilon 60^{\circ} L$
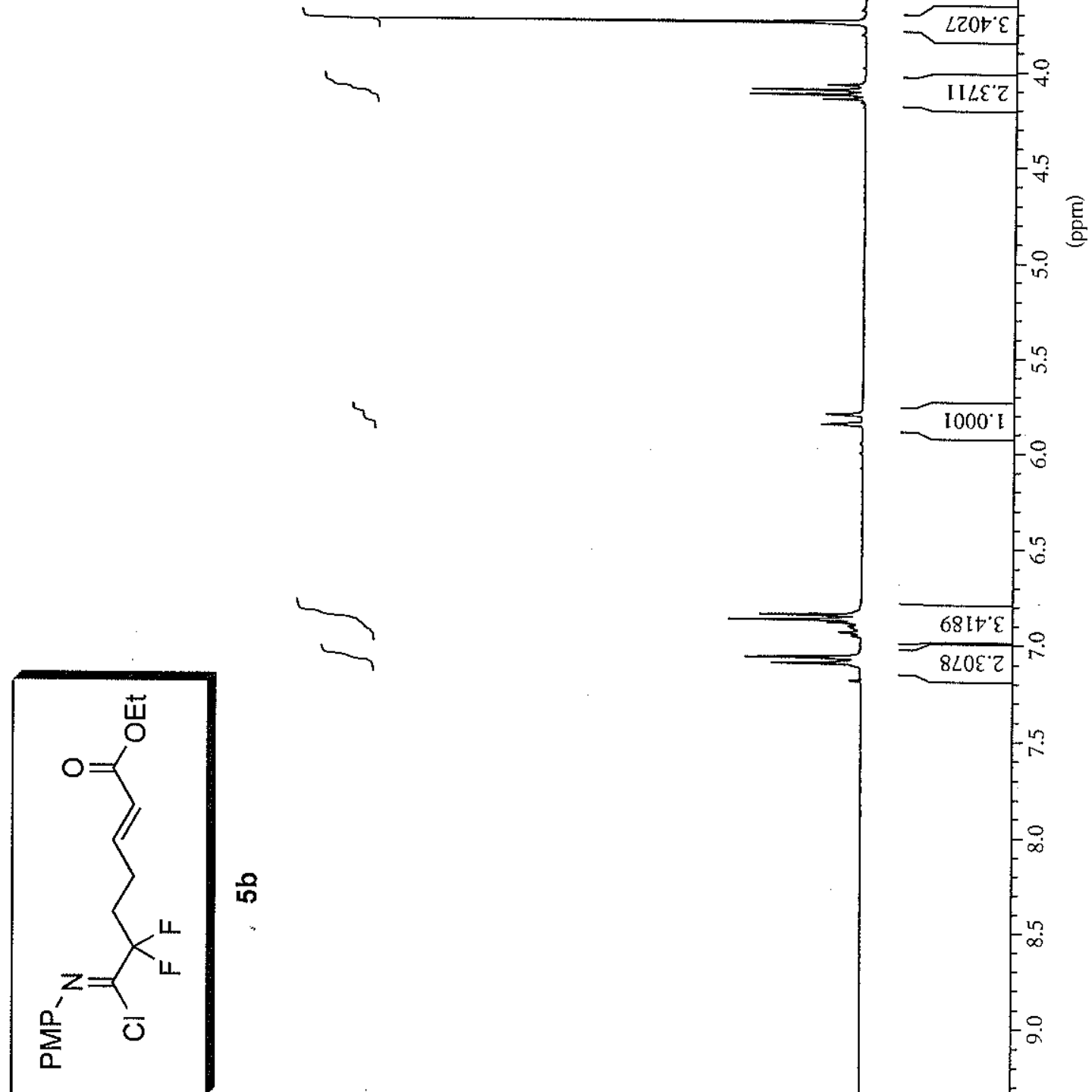

in

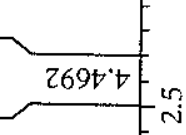

i

$m$

흥

in

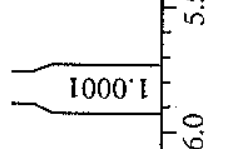

$-8$

?
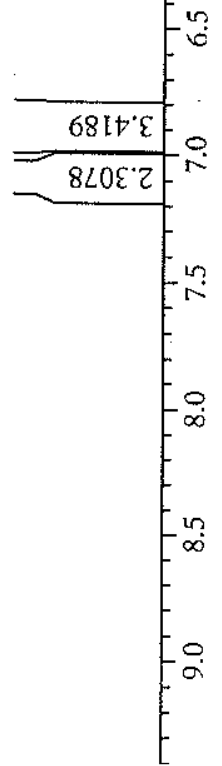

은

$\infty$ 


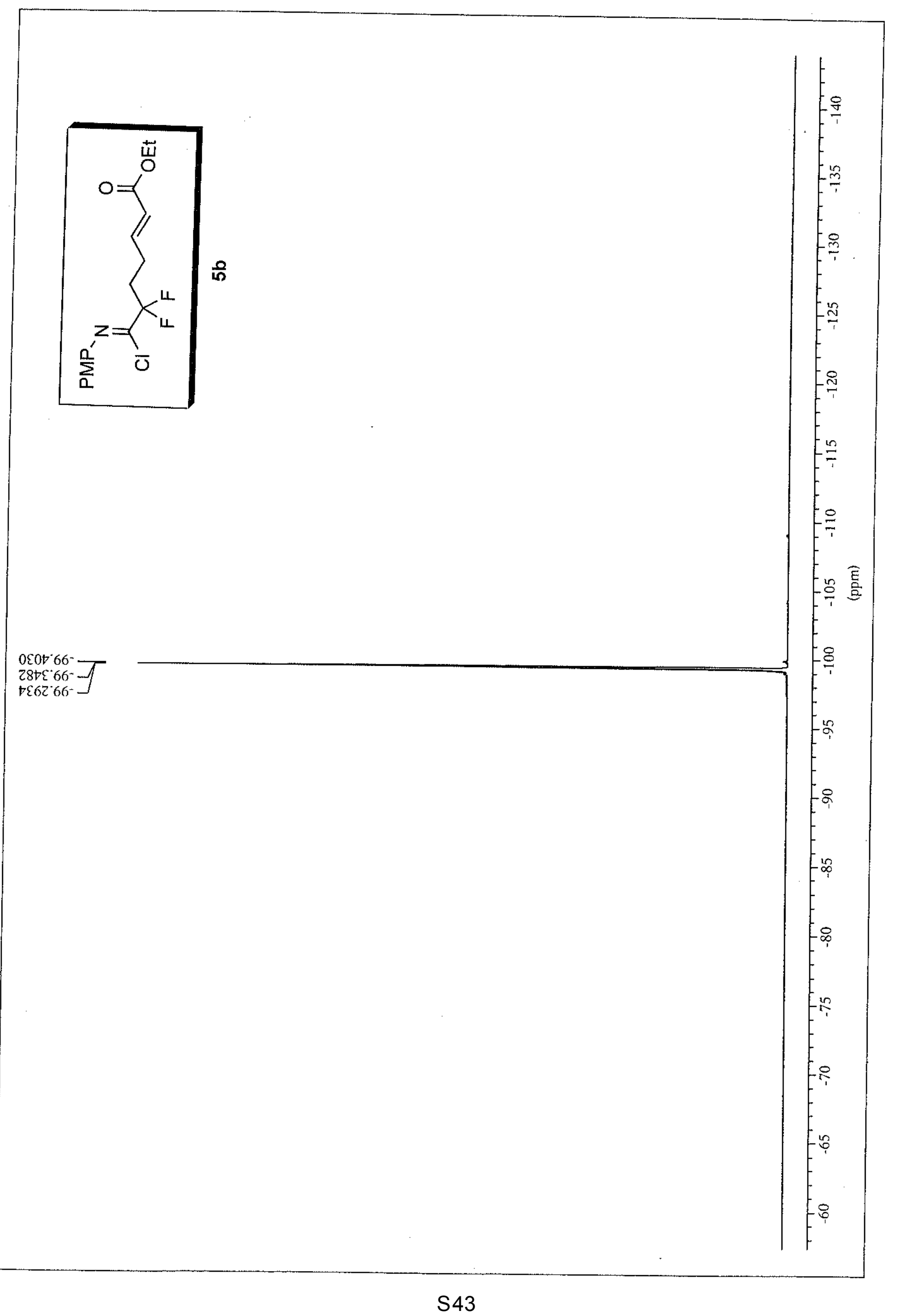




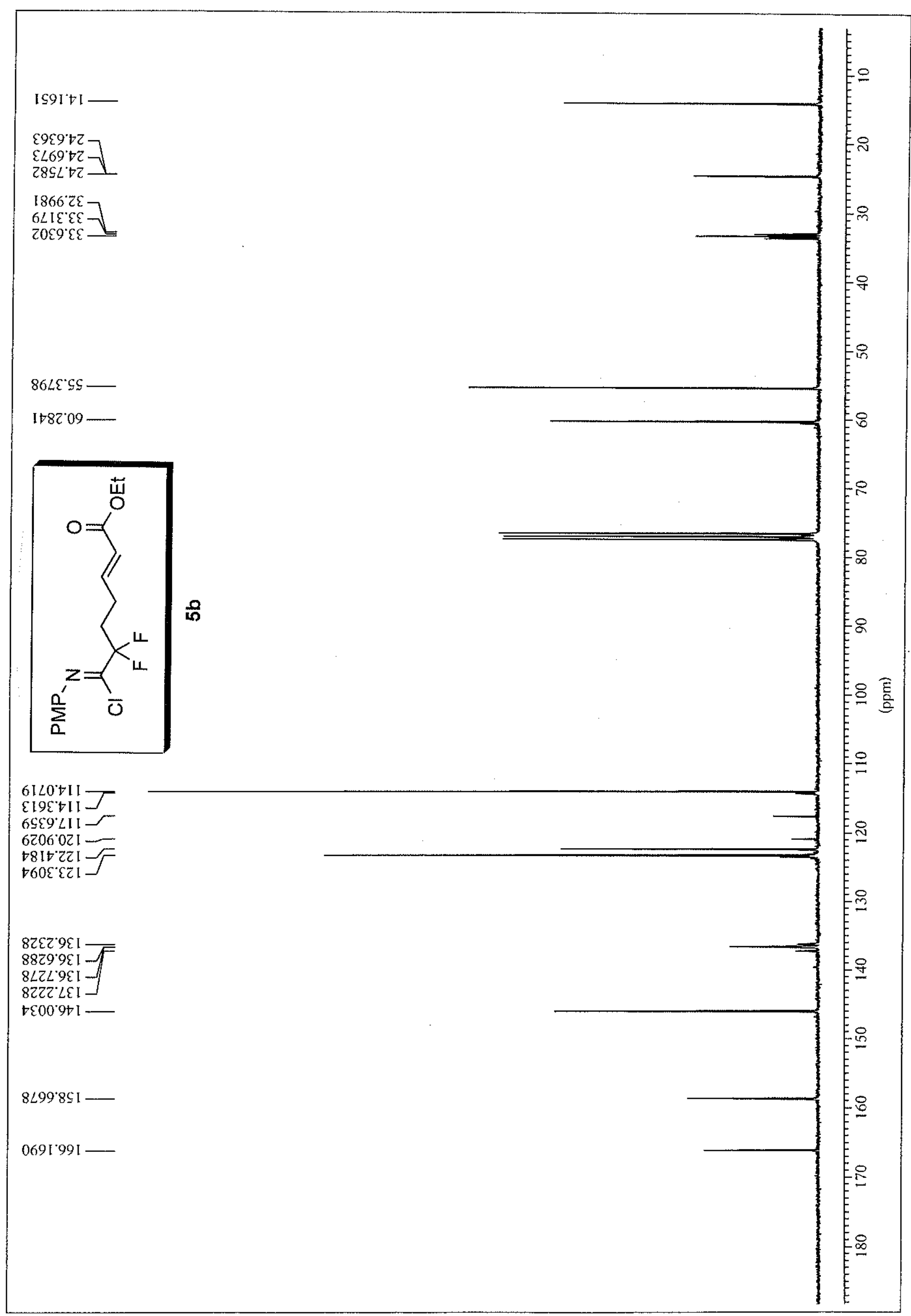


$088 \mathrm{I}^{\circ} \mathrm{I}$

6902' !

LOEZ' I

$\angle L 69^{\circ}[7$

$E Z L ' I$

$86 t L^{\circ} !$

$866 I^{\prime} Z$

$2602 ' 2$

$08 \mathrm{I} z^{\prime} \mathrm{Z}$

$\angle 82 Z^{\prime} Z$

ธZ\&Z゙

ZESZ' $Z$

†I8Z'Z

$I \mathcal{G} \leftarrow L^{\circ} \mathcal{E}$

\section{$\angle O \angle 0^{\circ}$}

$\$ 860^{\circ} \mathrm{F}$

$\checkmark 221 \circ-$

29t15

$0 S 9 L ' S$

$00 L L S T$

OSLL'S

$0 \angle 18^{\circ} \mathrm{S}-$

$1228^{\circ} \mathrm{S}$

$\left[\angle Z 8^{\circ} \mathrm{G}\right.$

$68 \mathcal{8} 8^{\prime} 9$

0698.9 庠

2LL8

$8668^{\circ} 9$

t226.

$5090^{\circ} \mathrm{L}-$

$6620^{\circ} L$
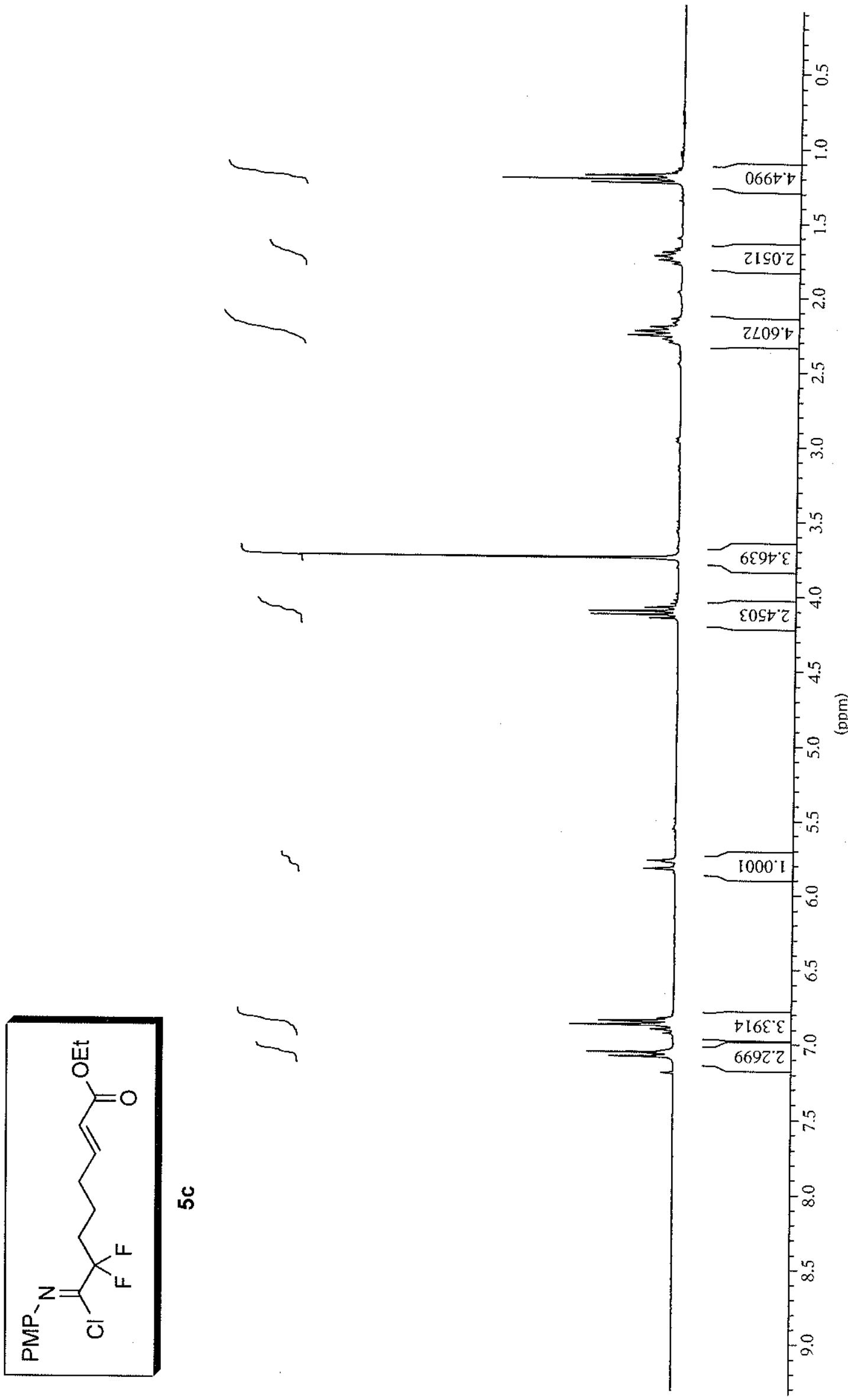


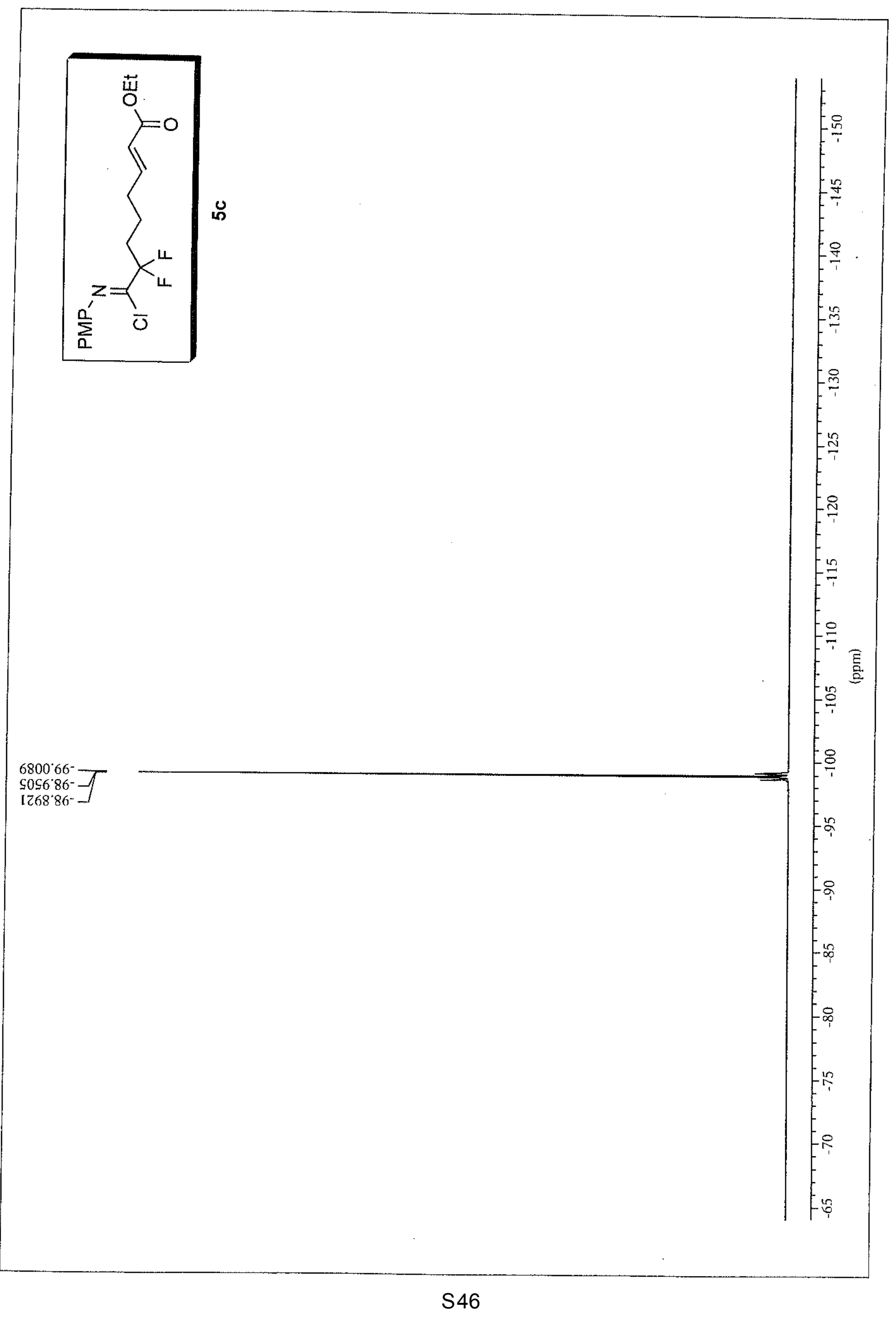




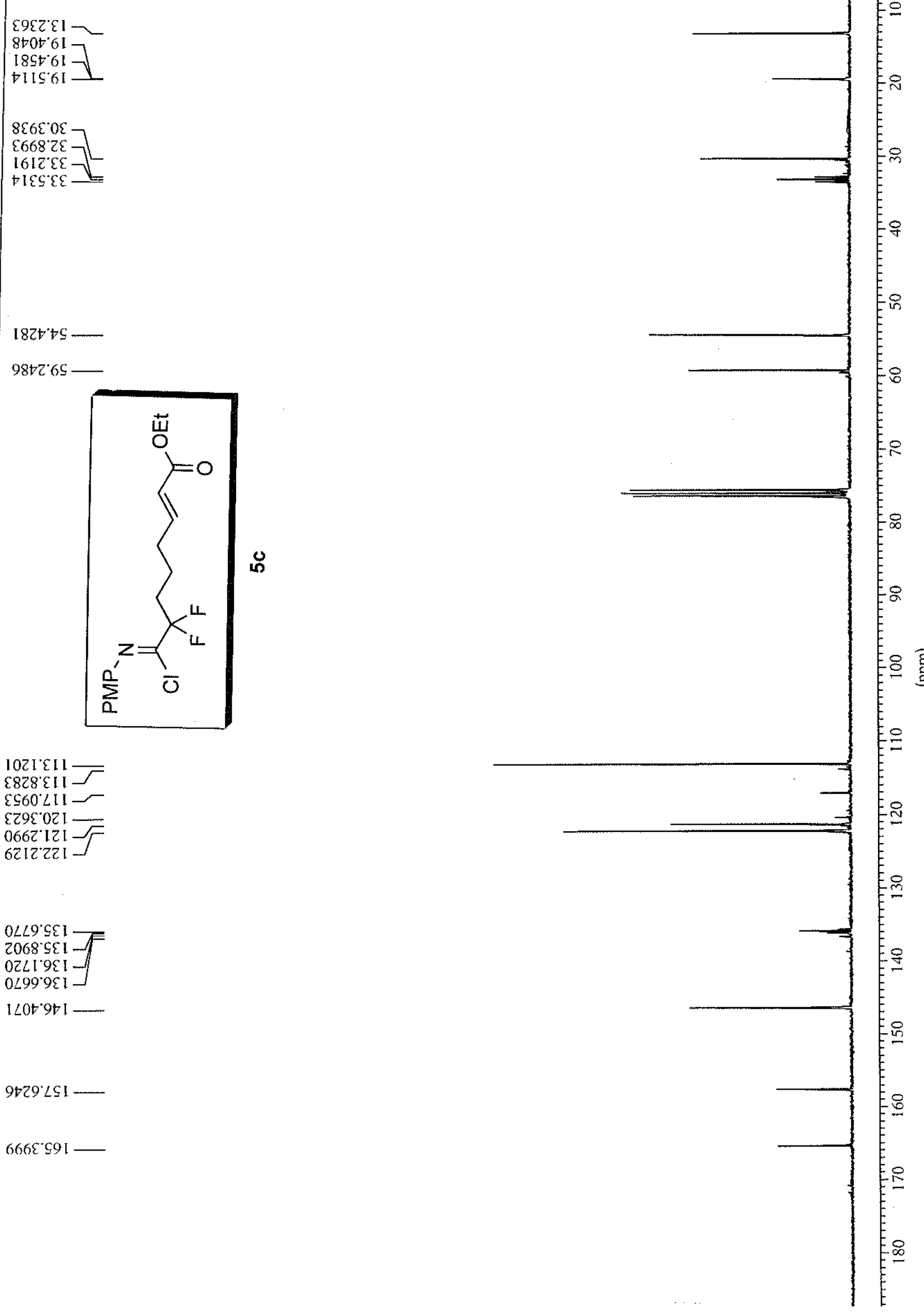




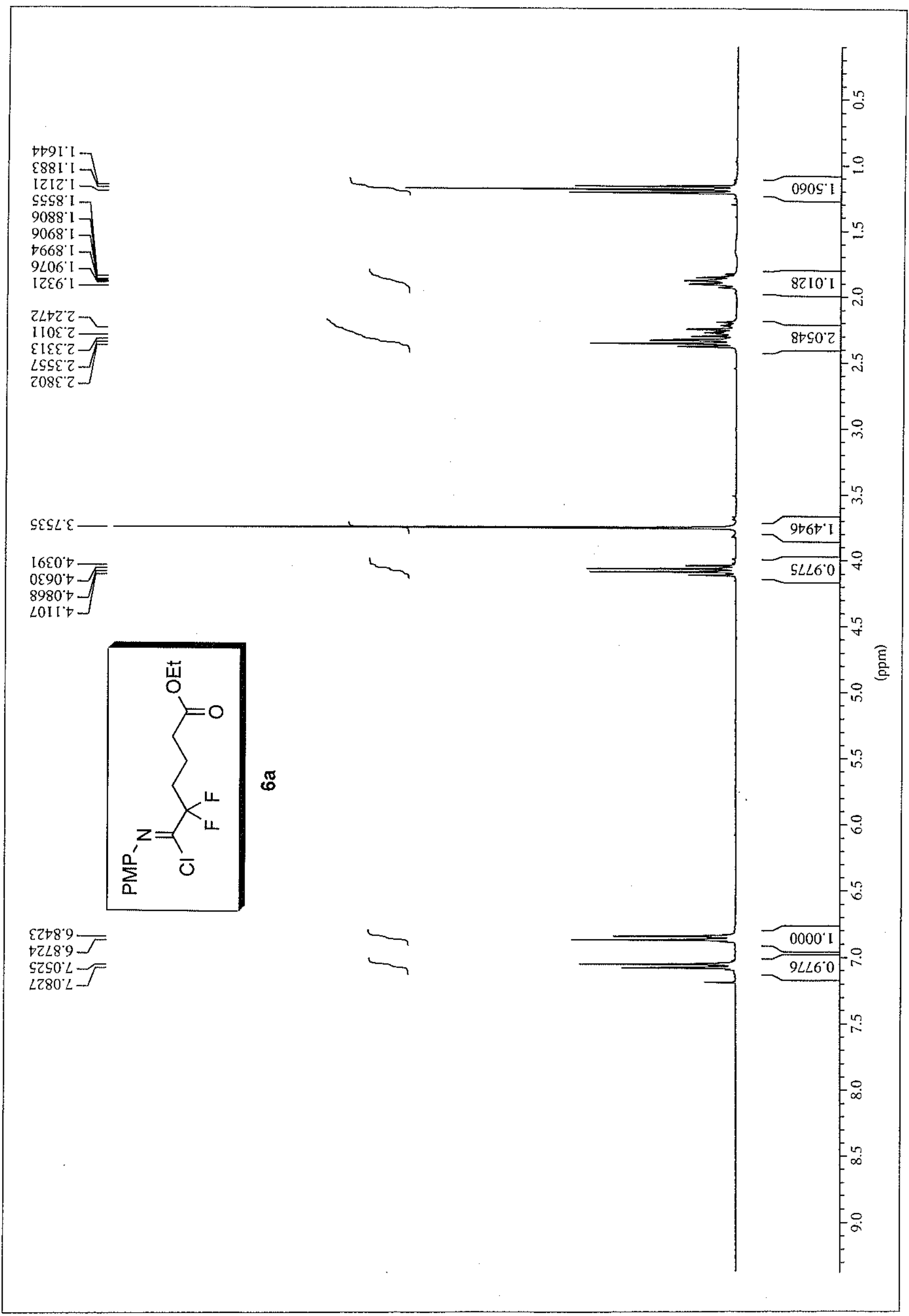




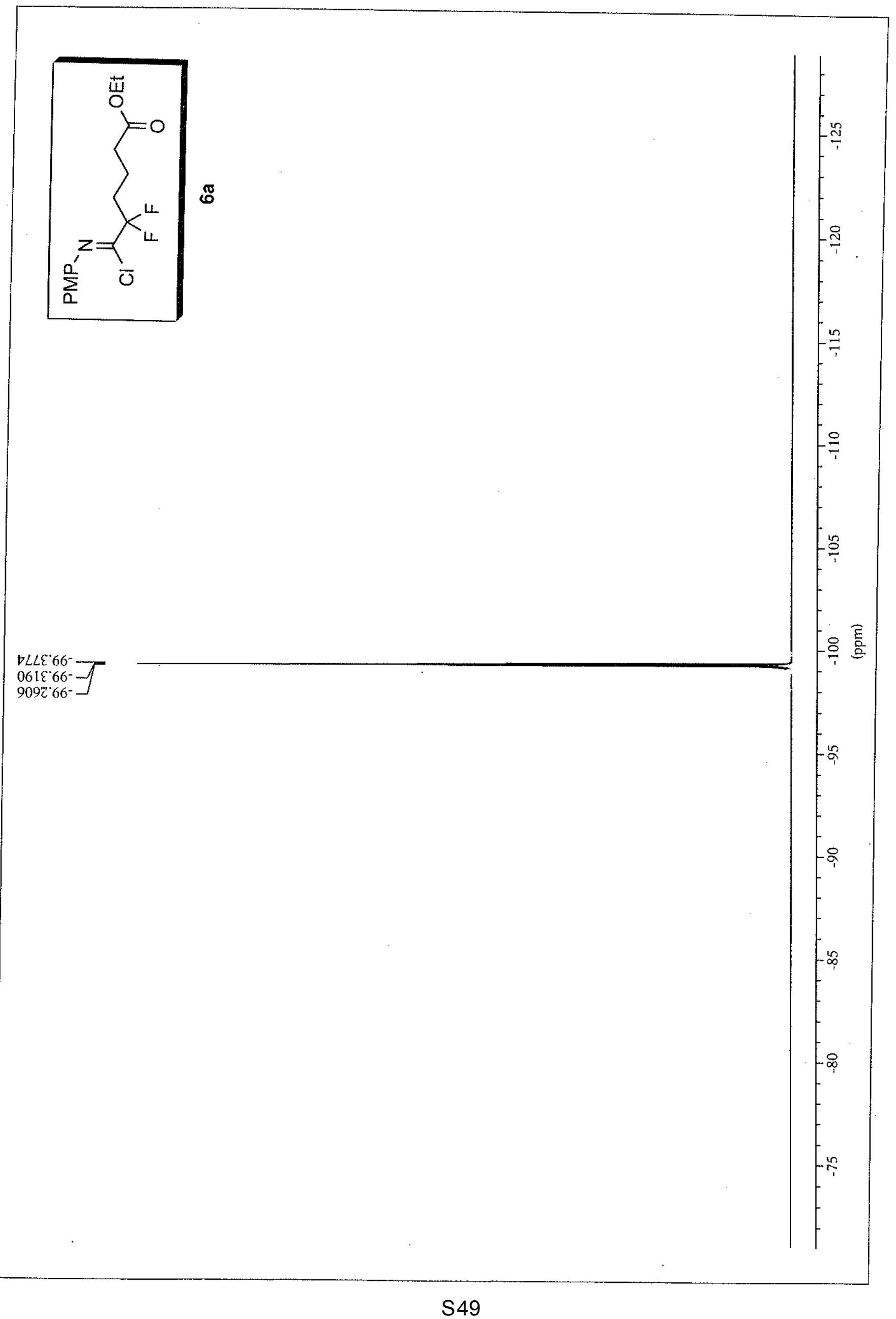




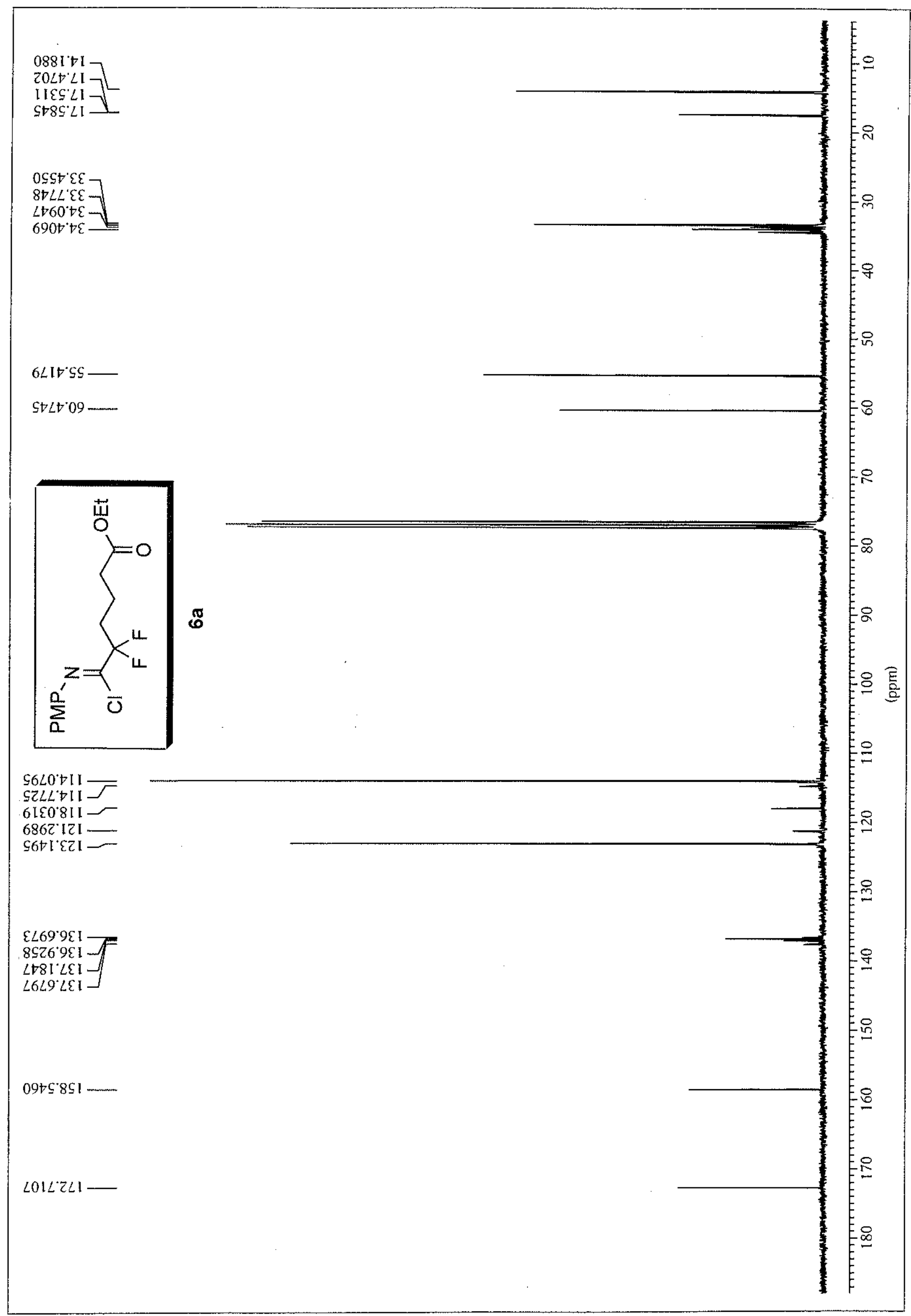




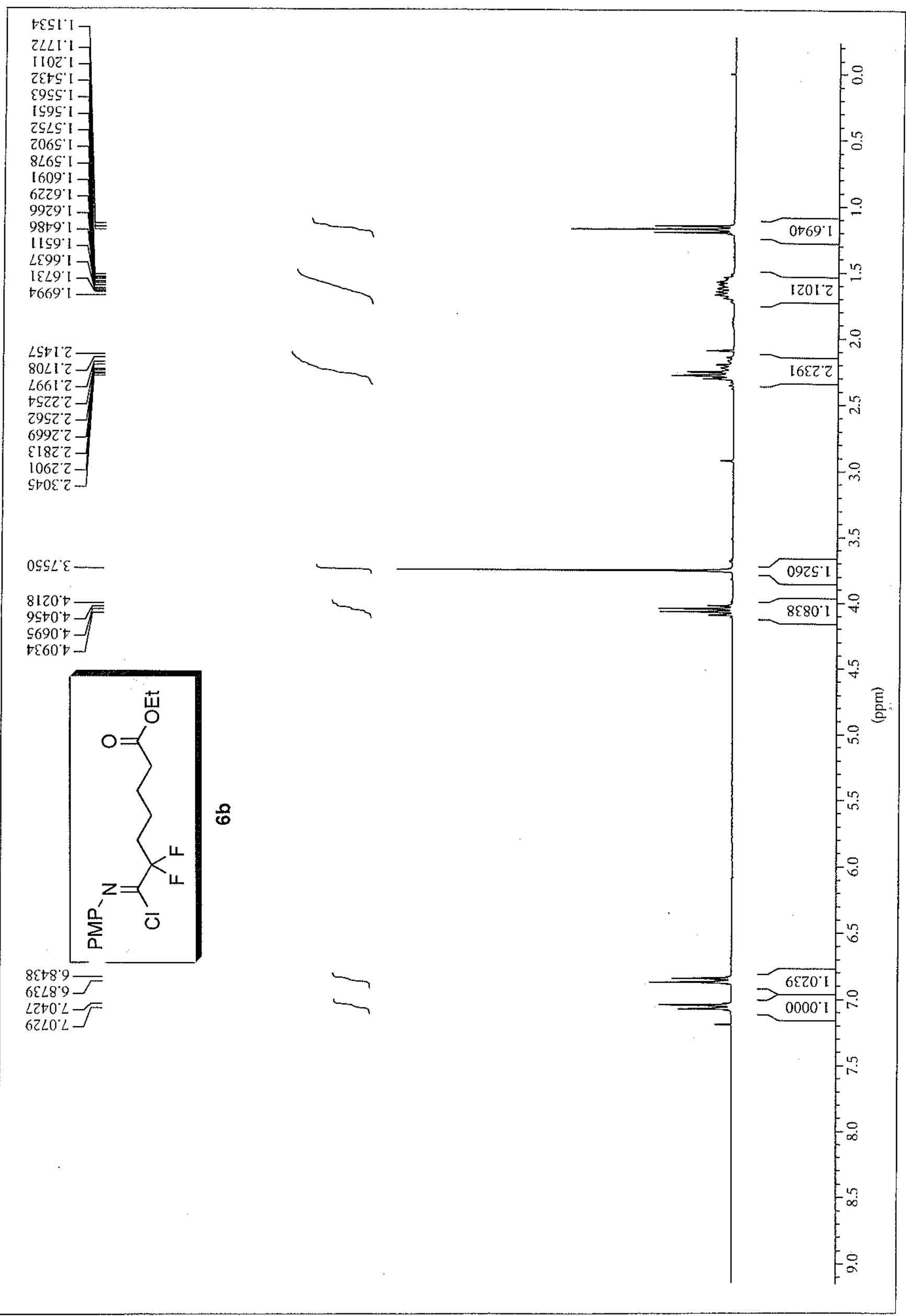




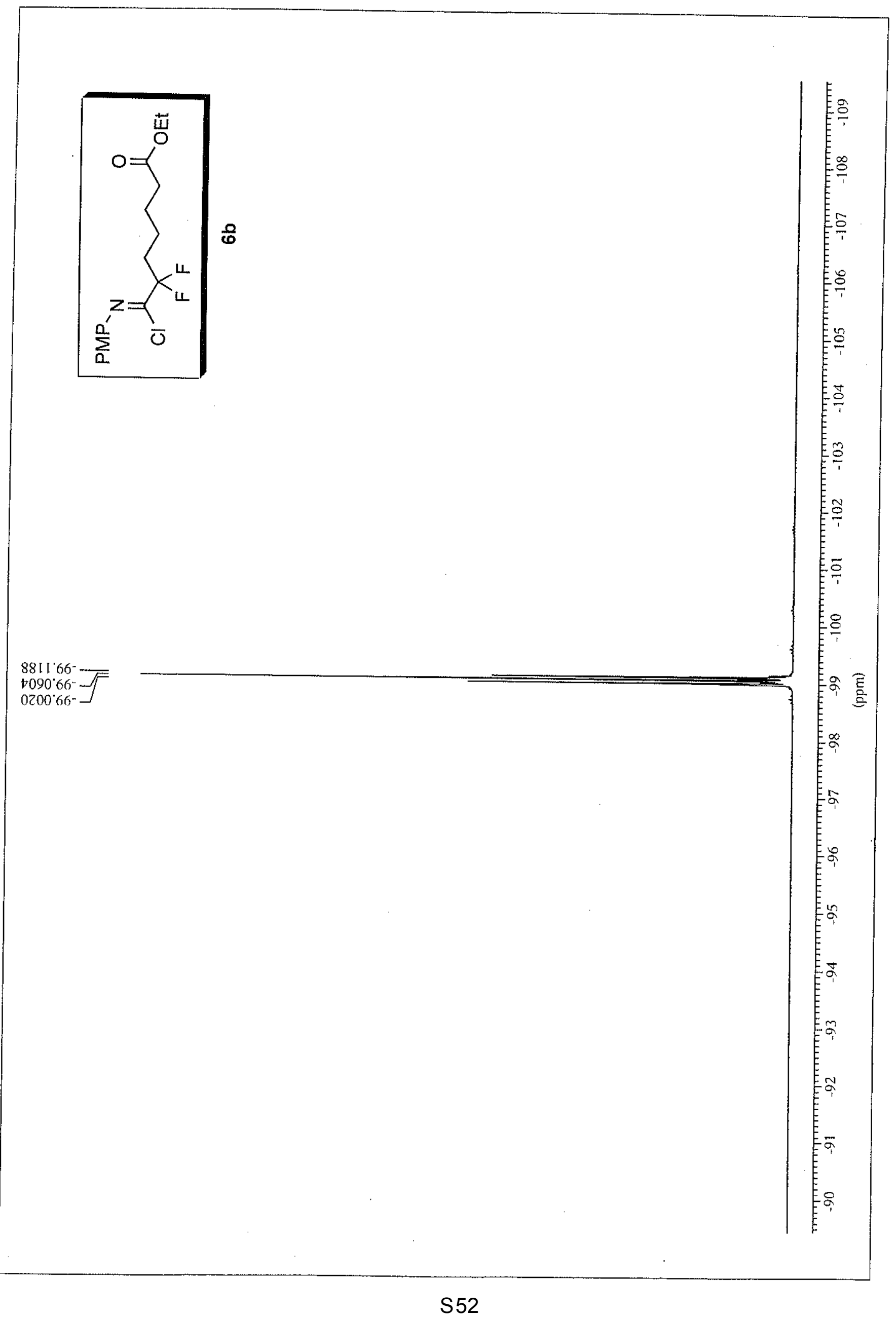


$088 I^{\circ} \mathrm{tI}$

$S t 8 \varepsilon^{\circ} I Z$
$6 \angle E \forall I Z$

Zl6t'I2

$889 \nabla^{\circ} \nabla Z-$

$8 \sqcup \varepsilon 6^{\circ} \varepsilon \mathcal{L} \neg$

S9l2

$8825+5-$

$98+8^{\circ} \circ \varepsilon$

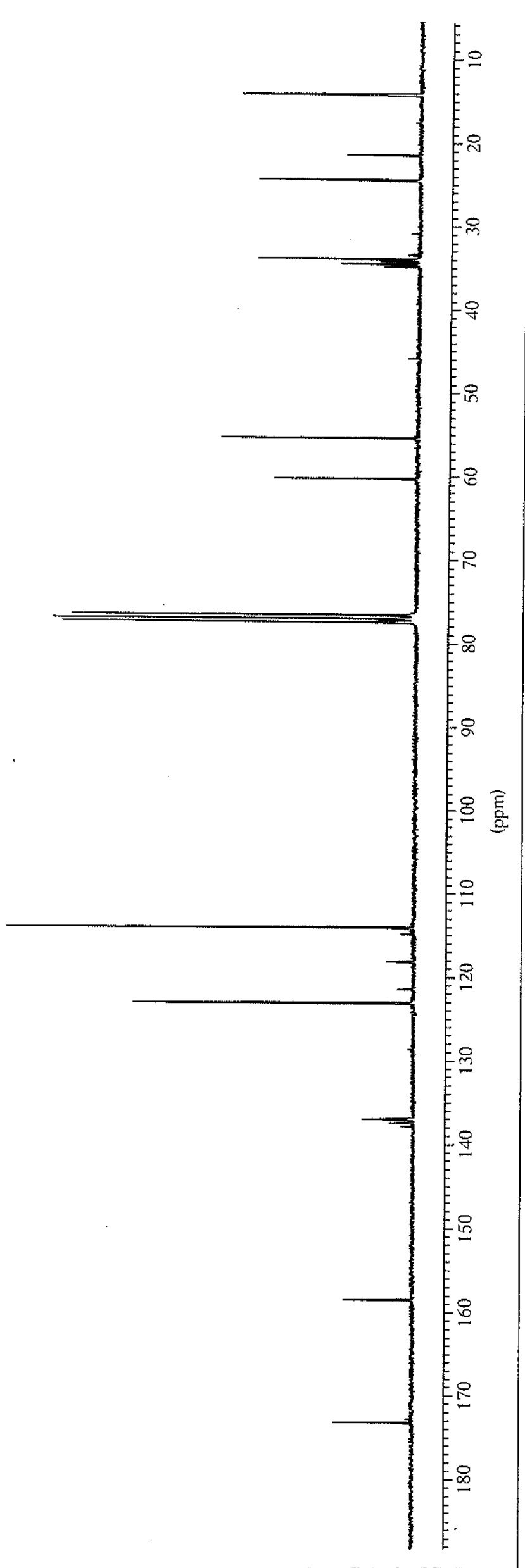

S6L0II

6C98'tII-
EEZI $811-$

ع06ElZl-

$8+98^{\circ} 9 \varepsilon \mathrm{I}$

$\angle 986^{\circ} 981-$

66کЕ'LEl

Z $\left.\angle P 8^{\circ} L E I\right]$

$6 \angle 0 S^{\circ} 89 \mathrm{I}-$

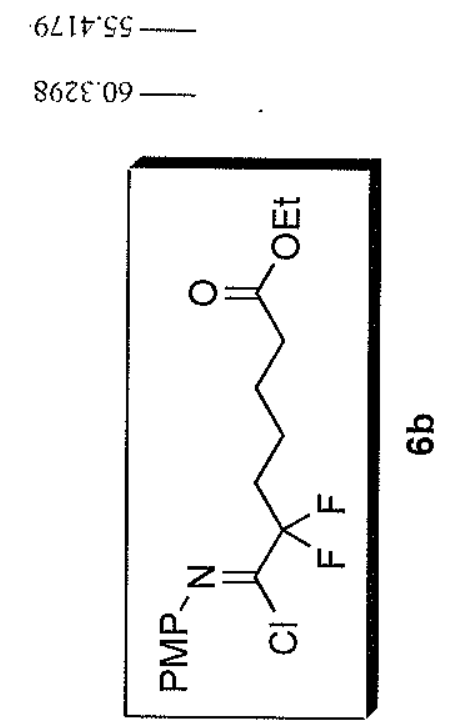

อำ

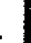

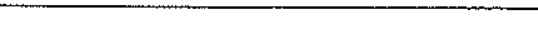

2

\$880'ㄱ1 -

F06 ${ }^{\circ} \mathcal{E L I}$ 


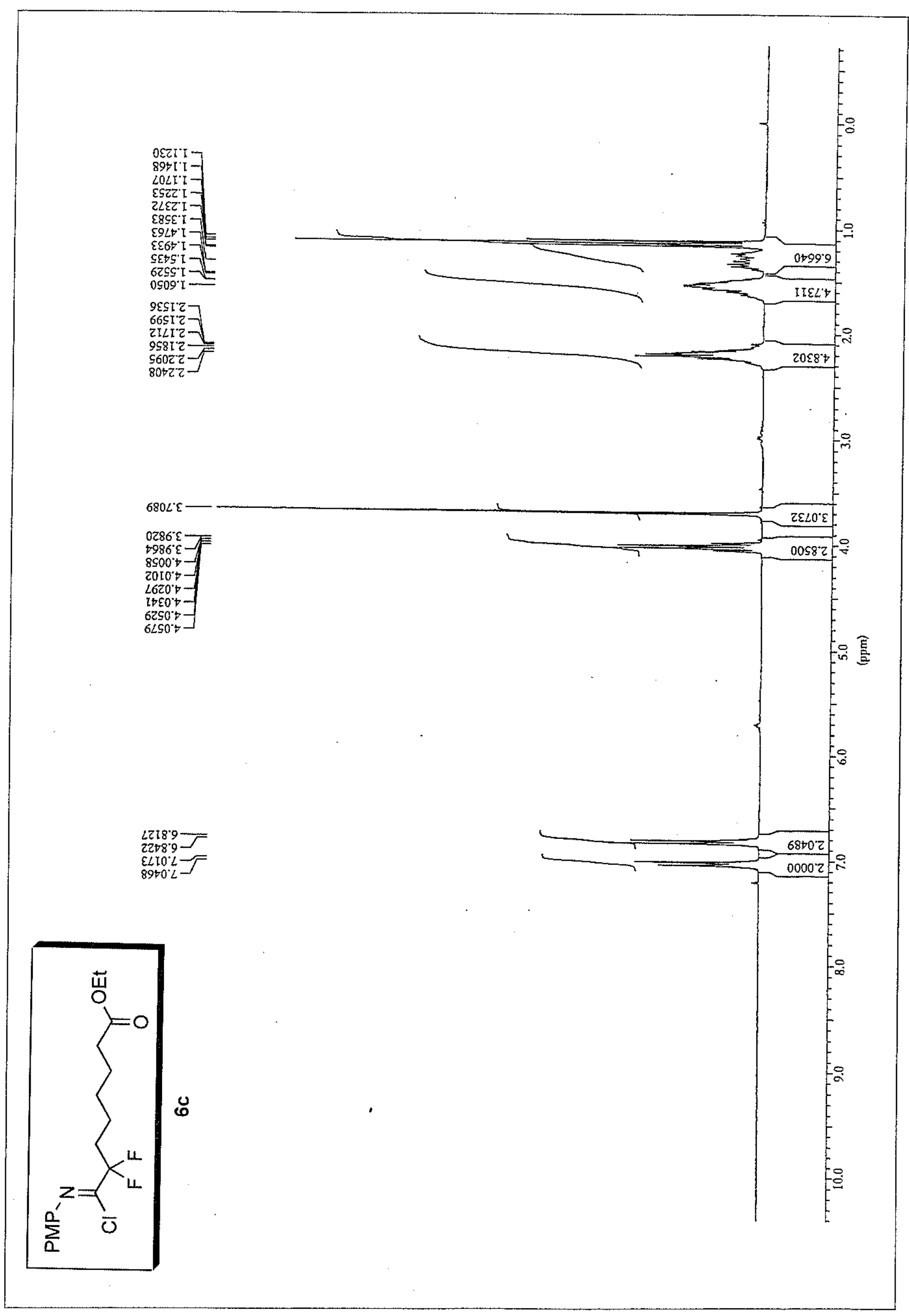



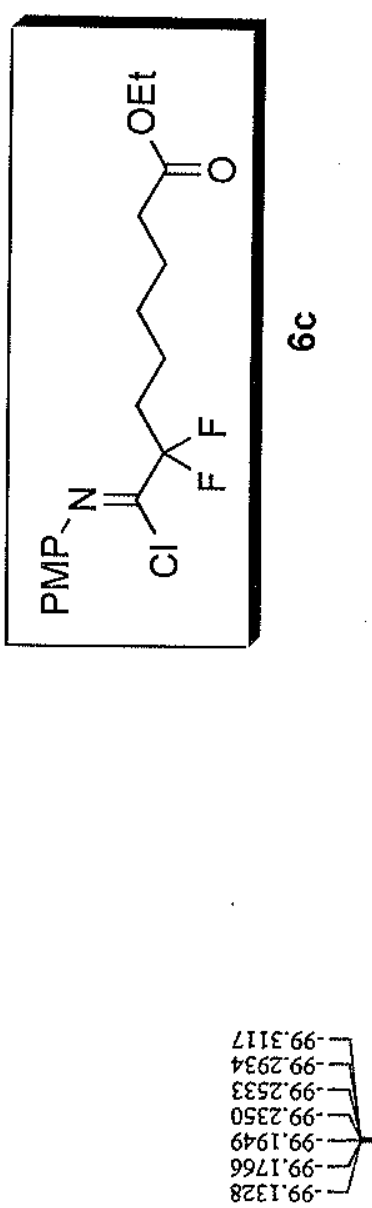

82LI ' $66^{-}$

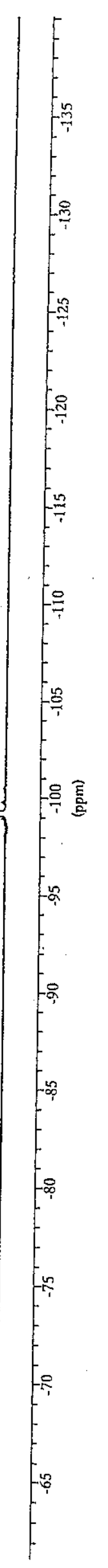


$\varepsilon 10 z+1$

szesiz-

$9 z \angle S^{\circ} \triangleright Z$

$+91982$

$0 \mathrm{C}+0 \circ \mathrm{t}-\mathrm{C}$

$20 t \varepsilon+\varepsilon$

$0 \varepsilon \varsigma 9 \downarrow \varepsilon$

$\angle S 96+\varepsilon-$

$\nabla L I T S S$

$\angle 9+209$

$6185^{\circ} 9 \mathrm{~L}$

$\angle E 00 L L$

$\angle 880^{\circ}+11$

$\varepsilon \varepsilon 86^{\circ}+11 \ldots$

68727811

zLOS $|z|-$

$16+0 \varepsilon z 1=$

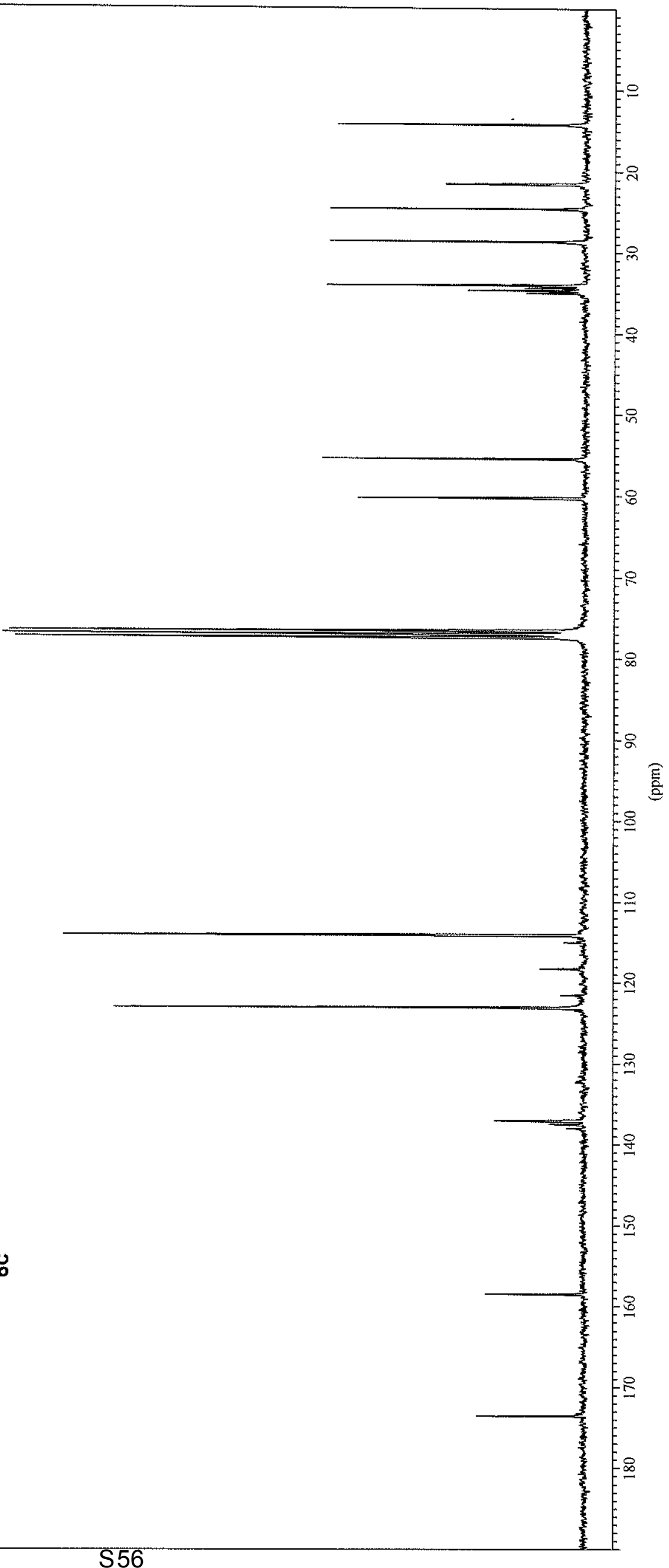




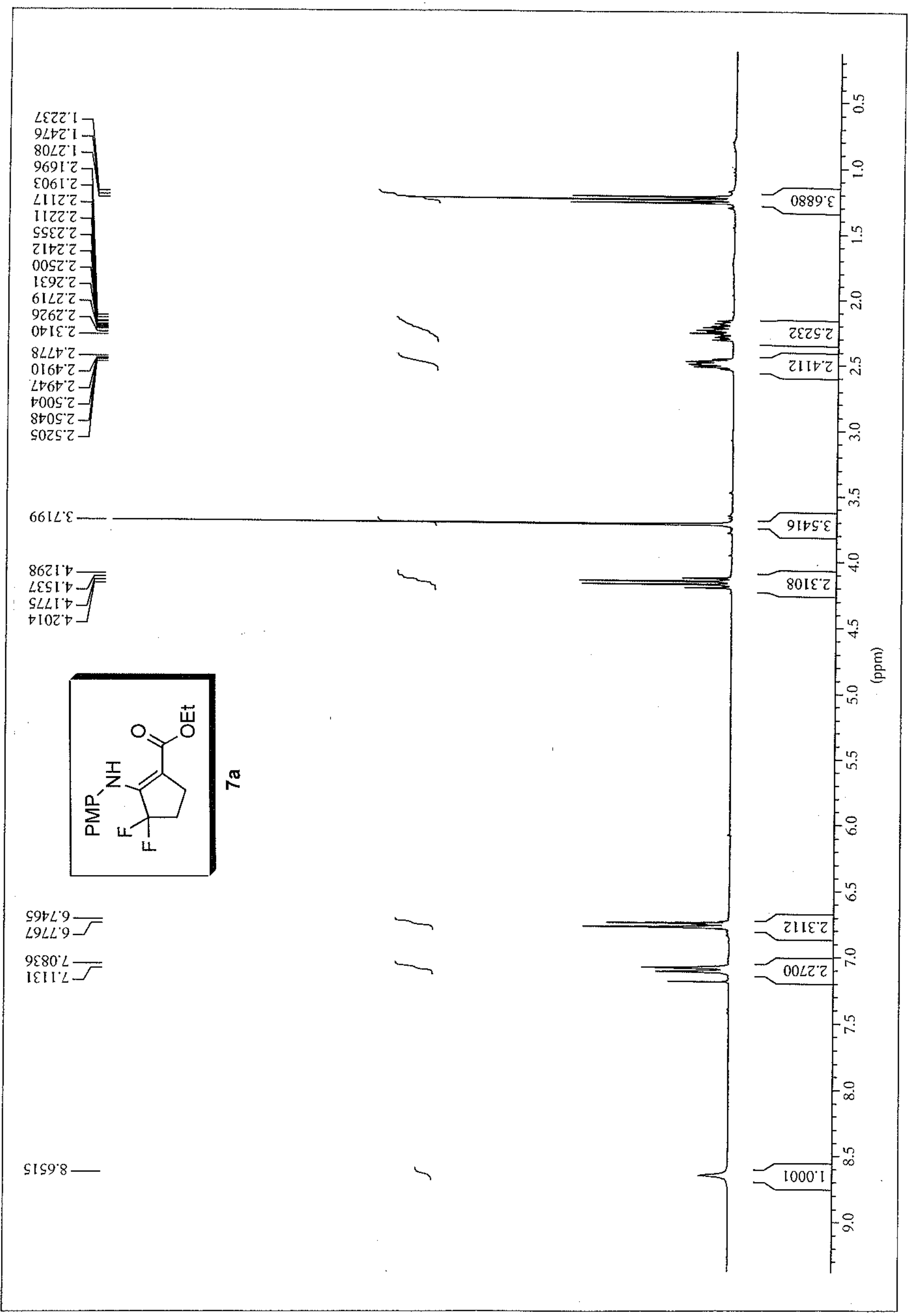




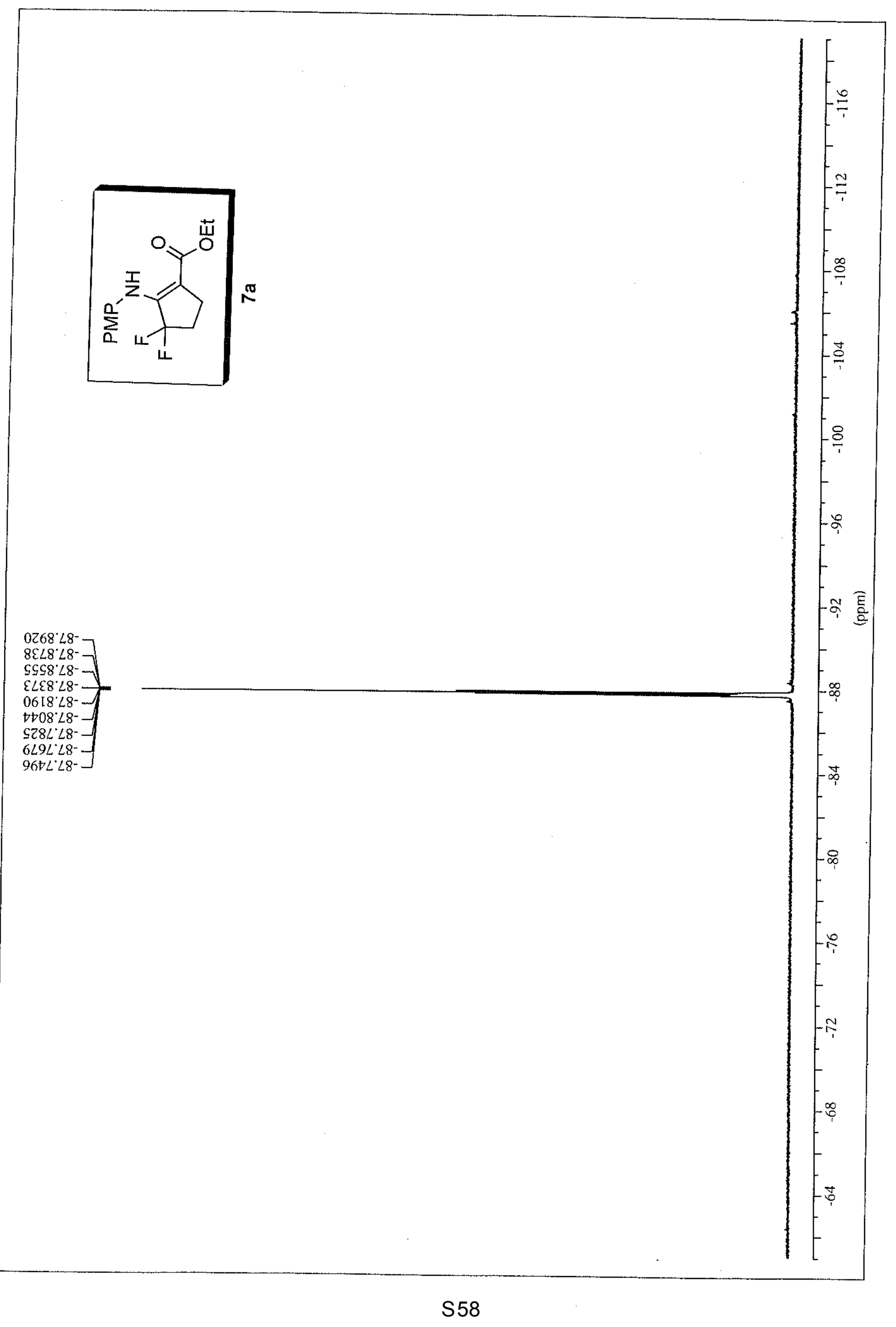




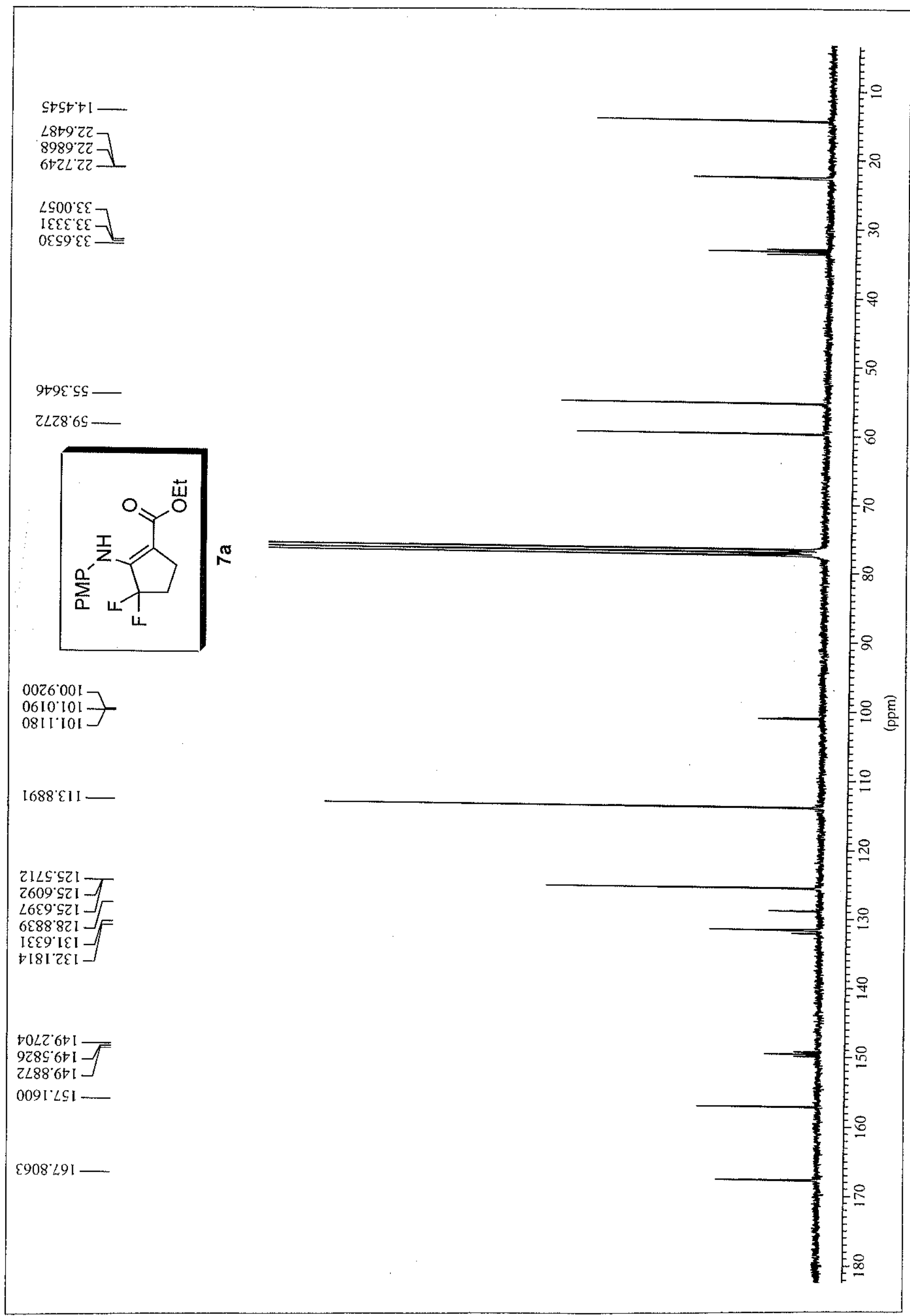




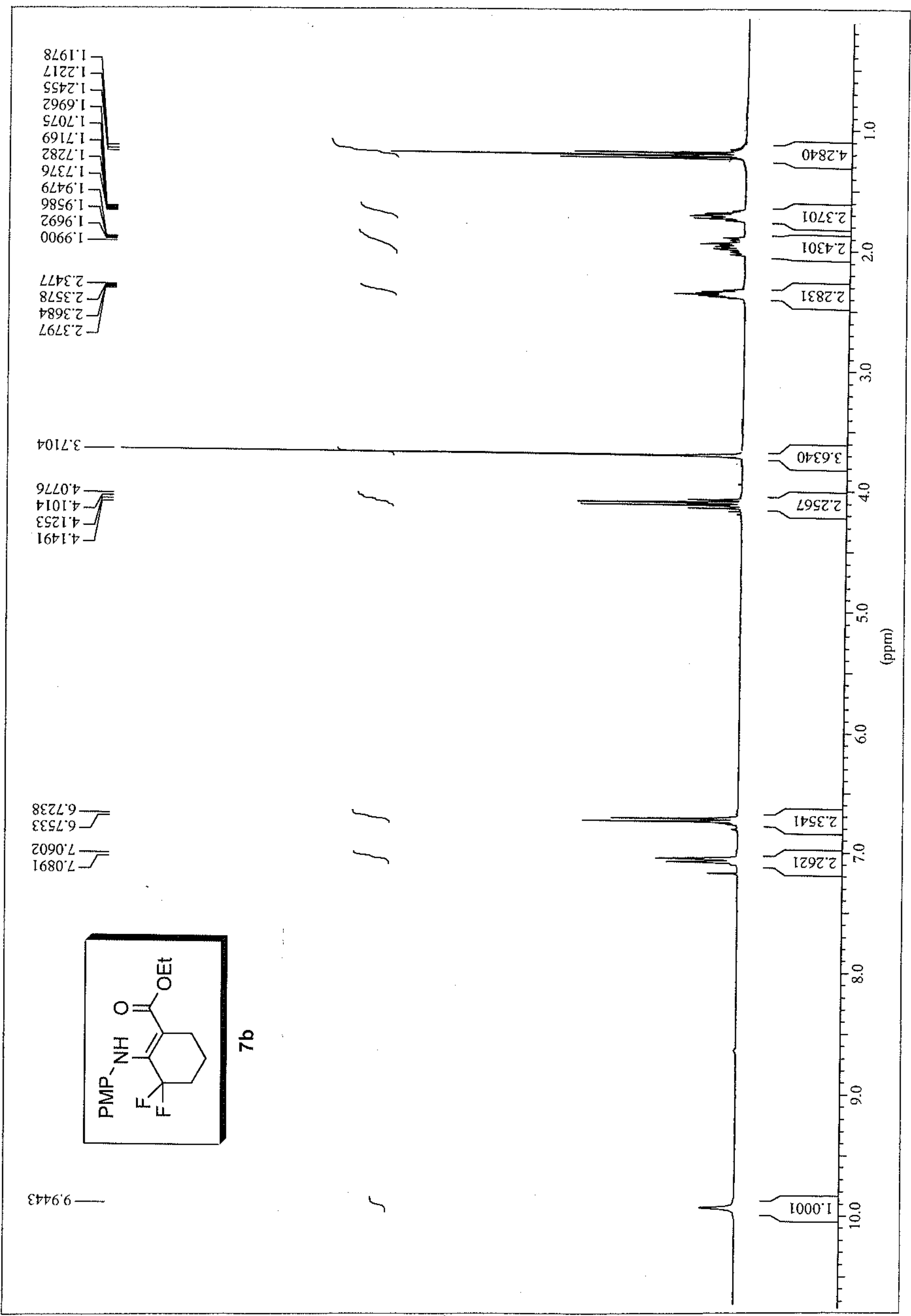




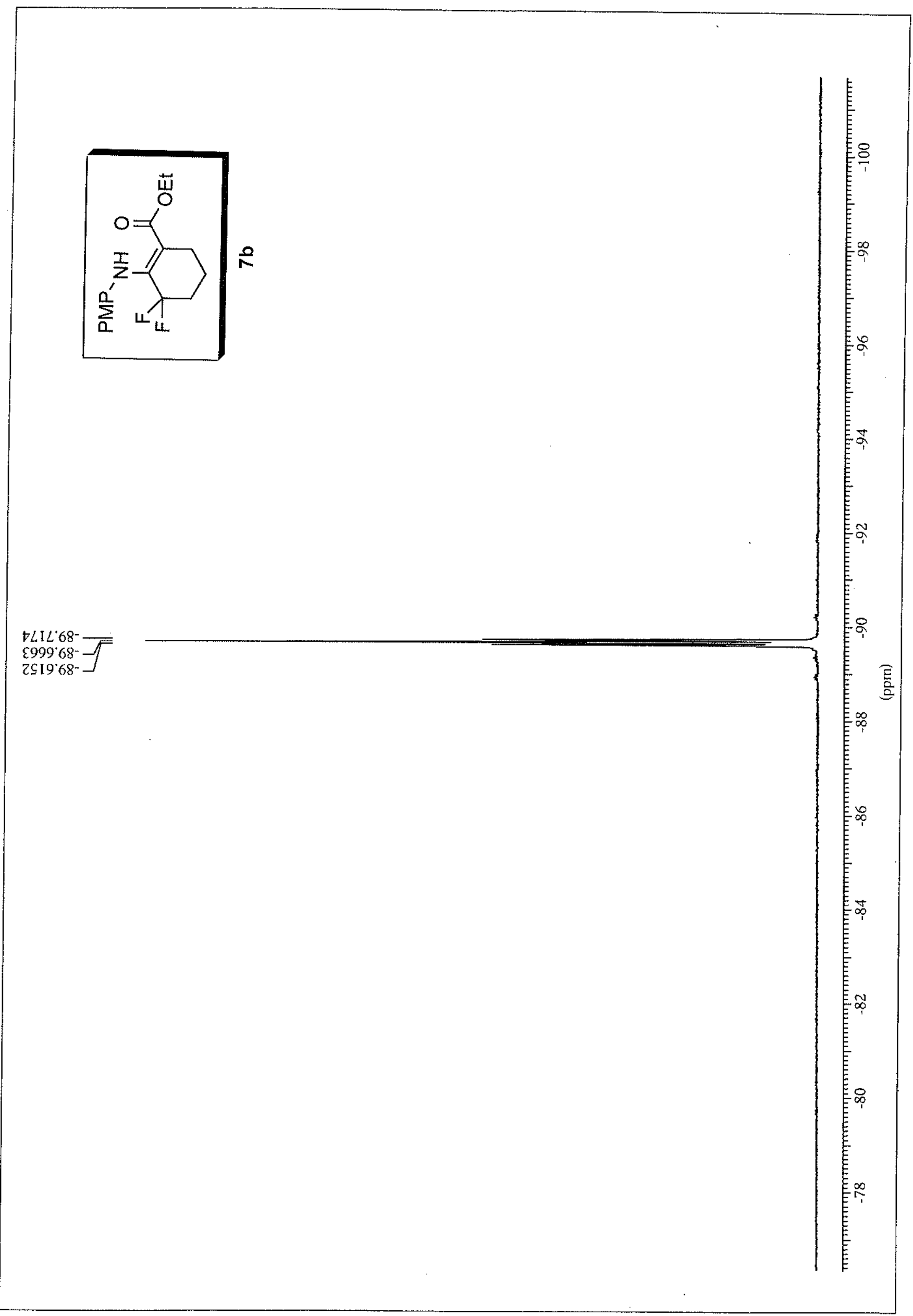




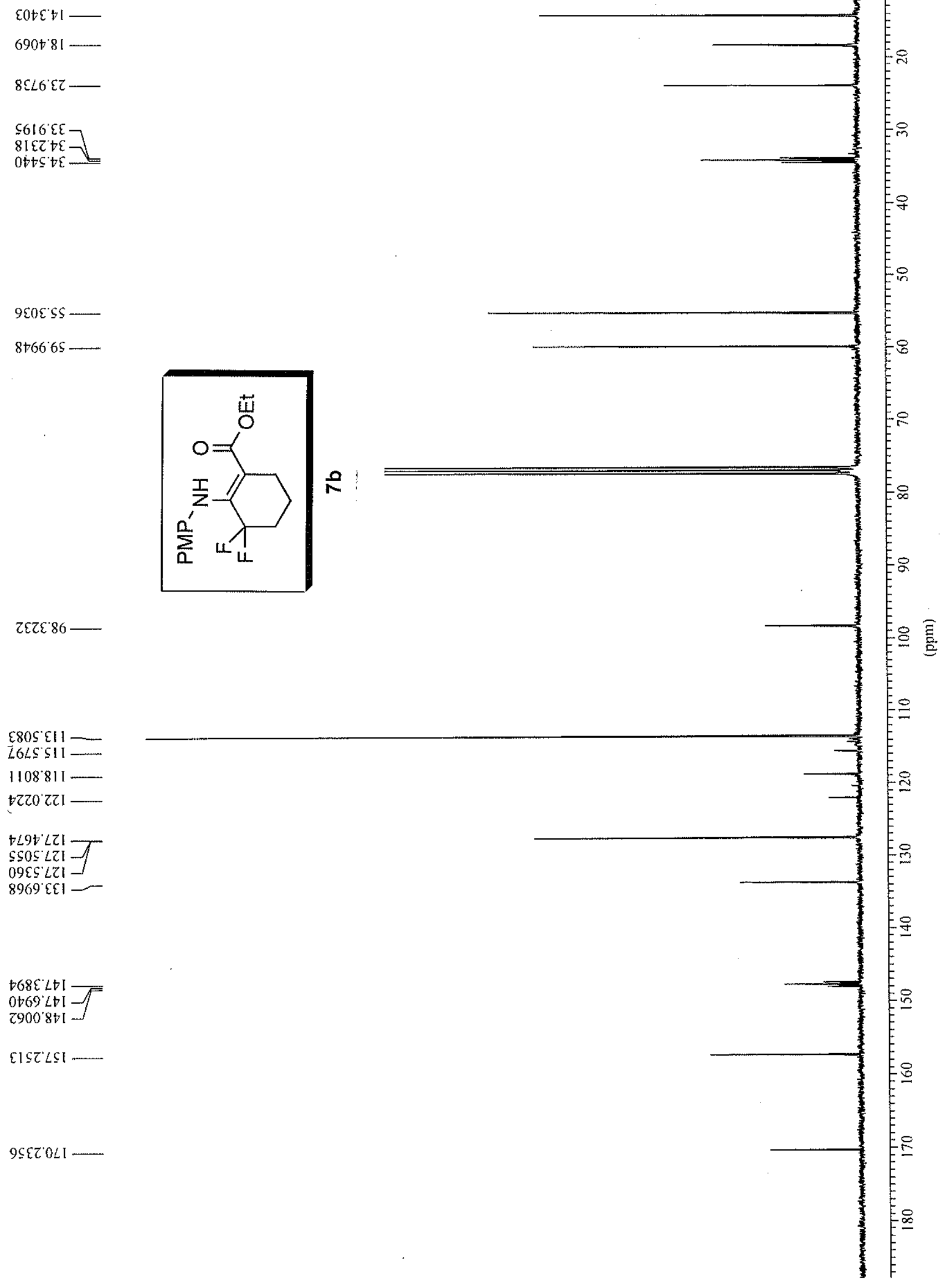




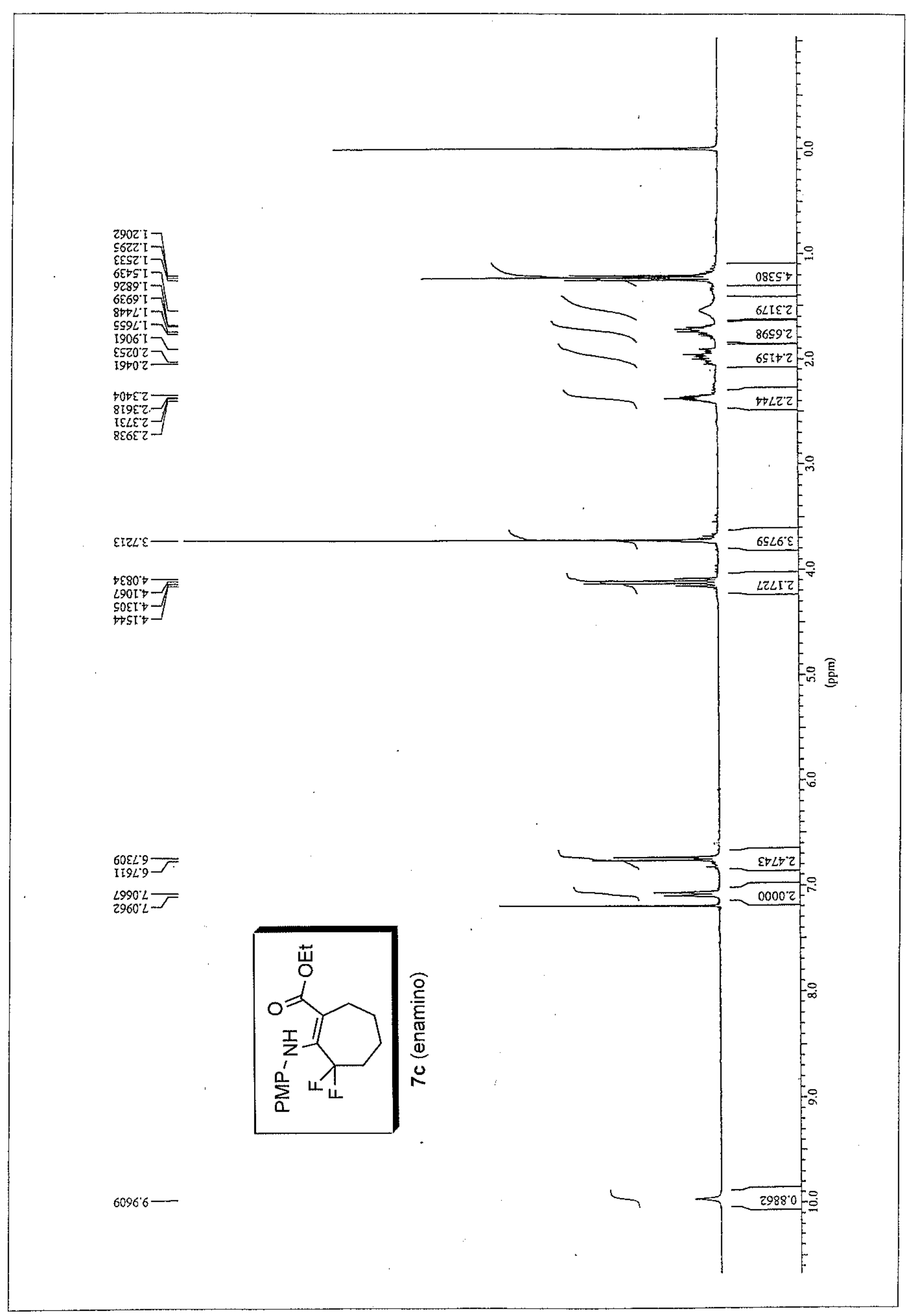



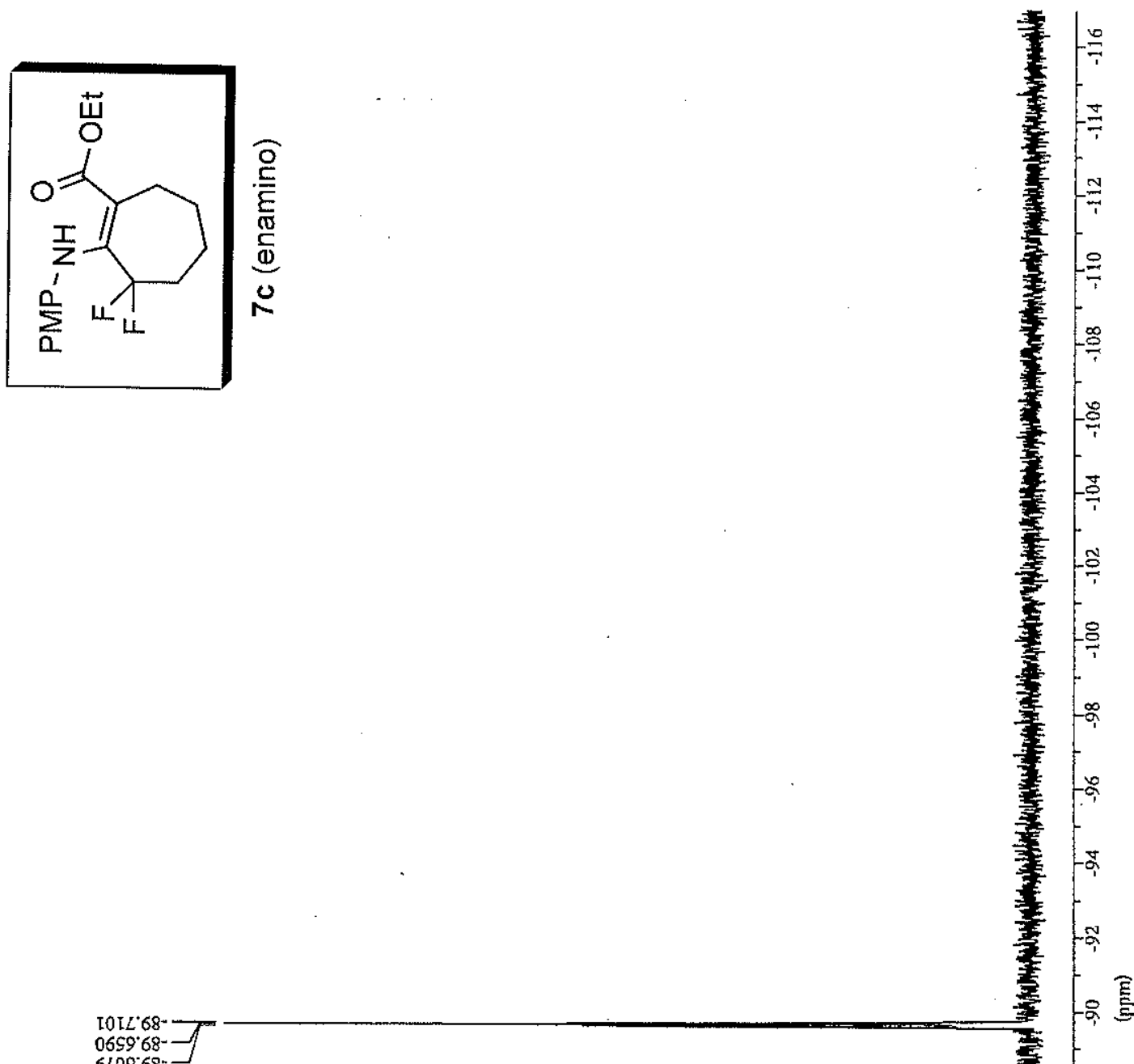

$6209^{\circ} 68^{\circ}$

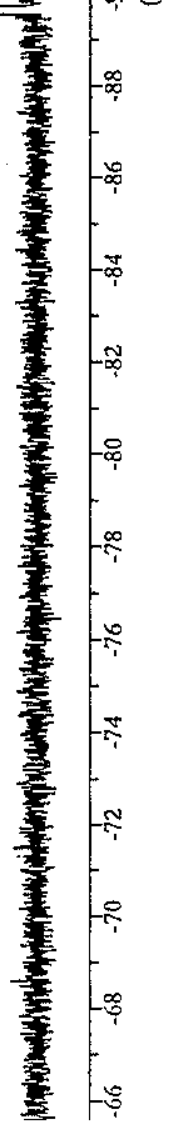




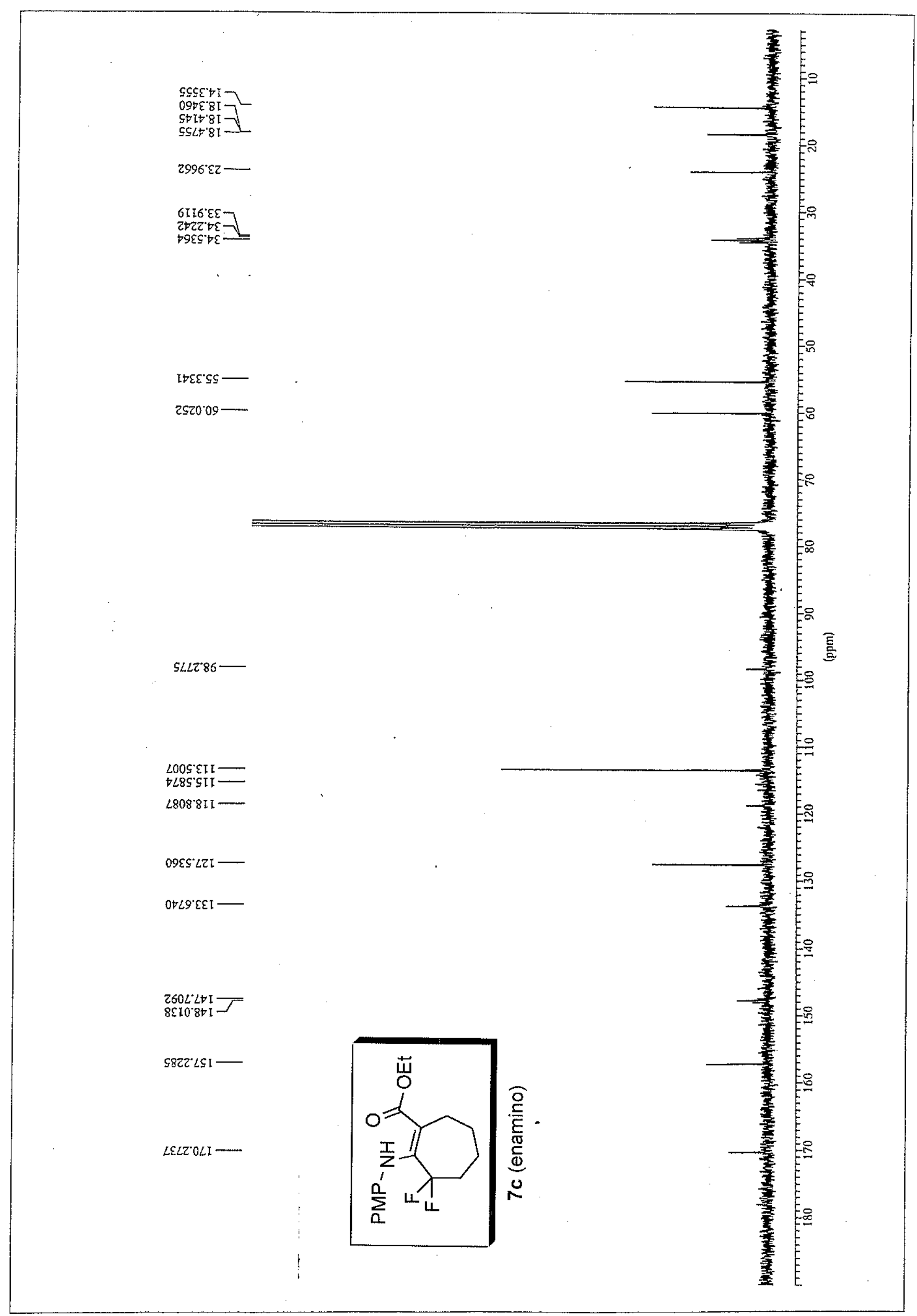




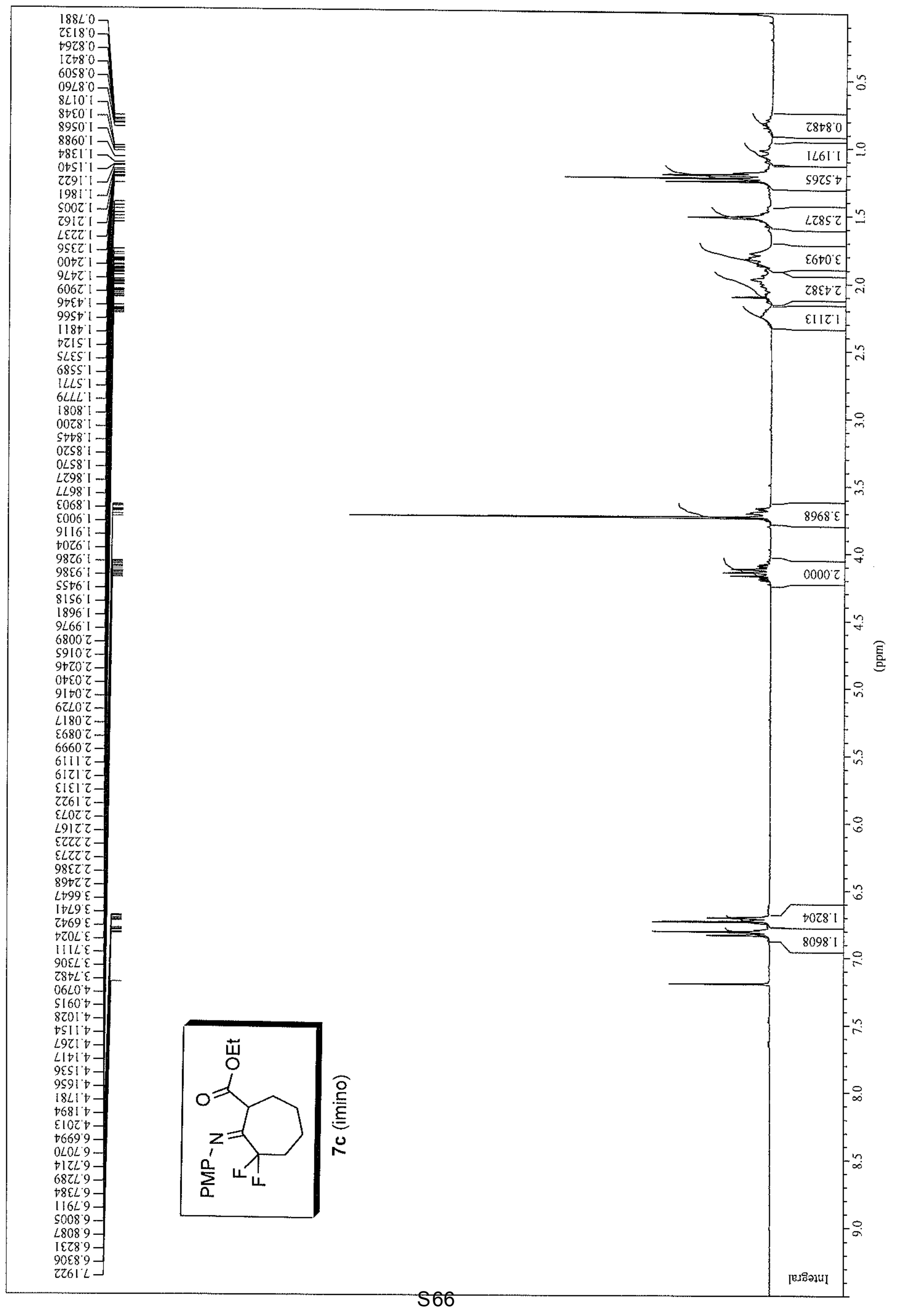


$2100^{\circ} 011^{-}$

E6L6601-

$\angle 088^{\circ} 601^{-}$

$8698601-$
$8858601-$

$\varepsilon \varepsilon+1601^{-}$

L8Z1 601 -

DIZI $601-$

8220601 -

$6000601-$

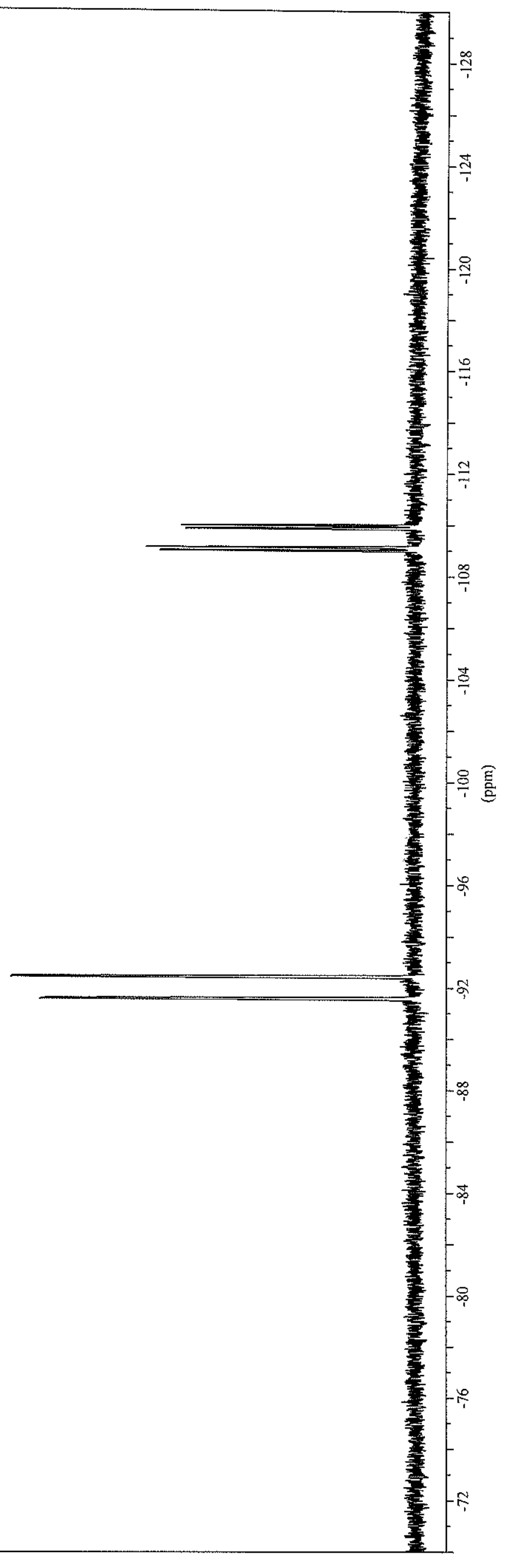




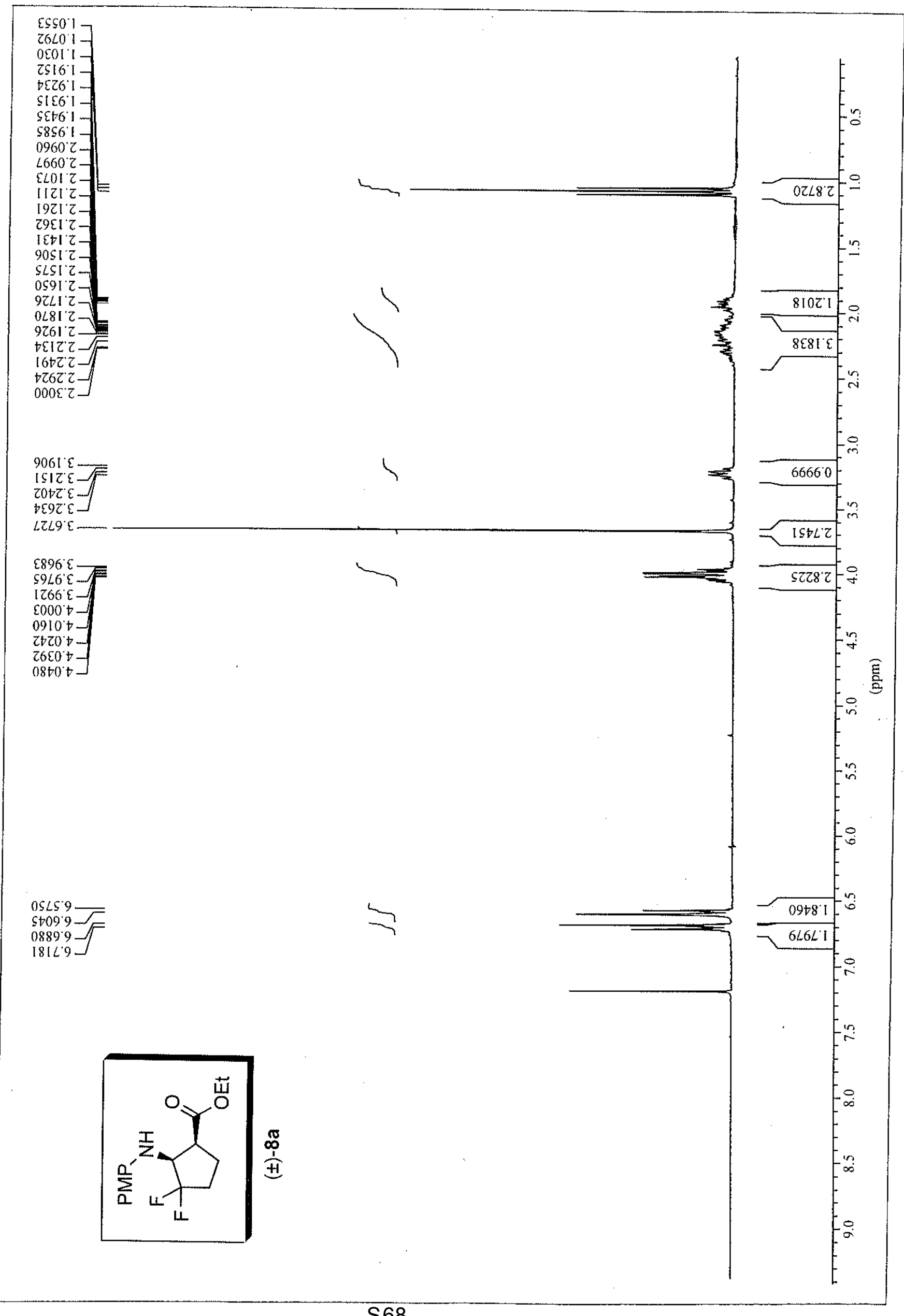




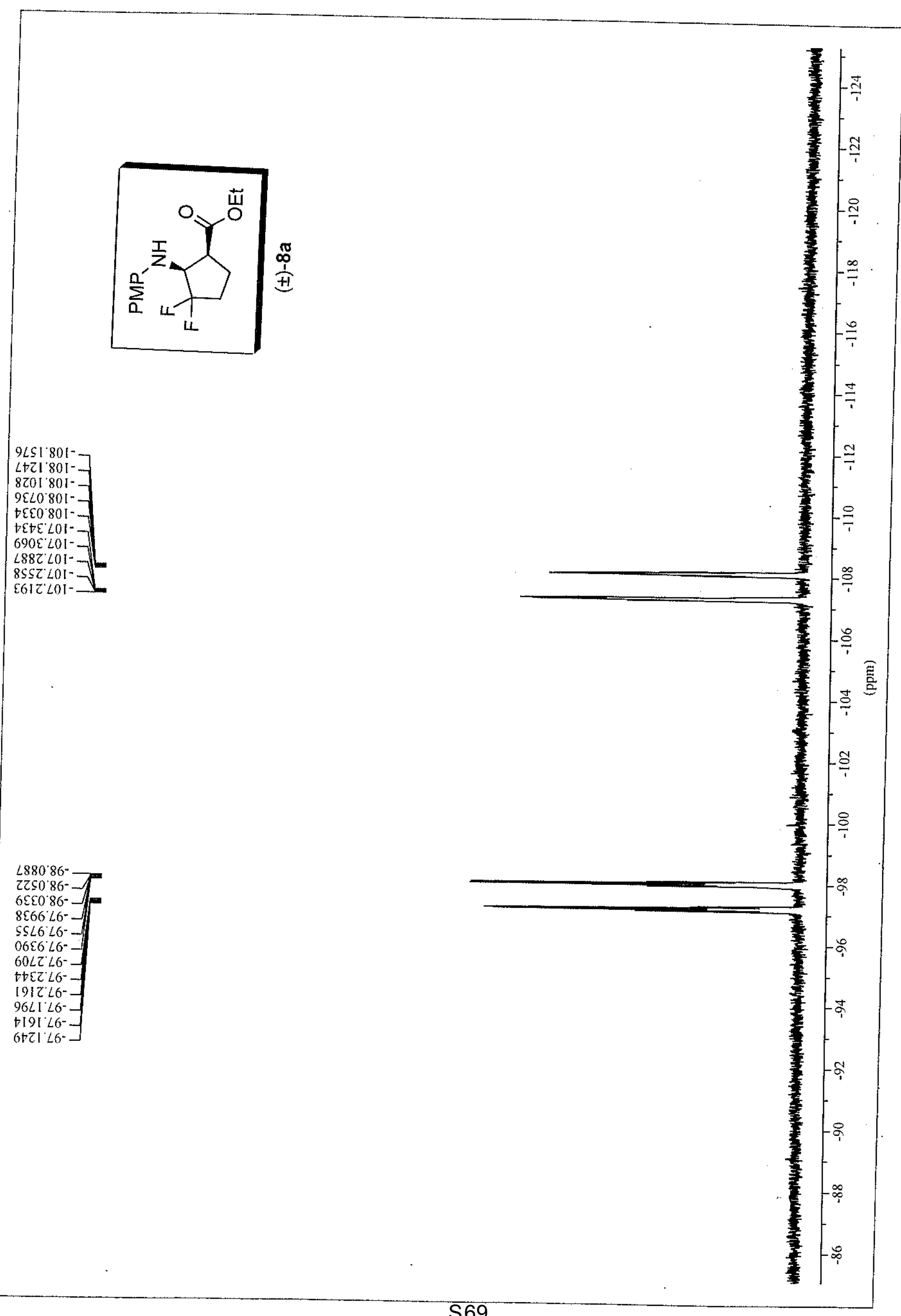




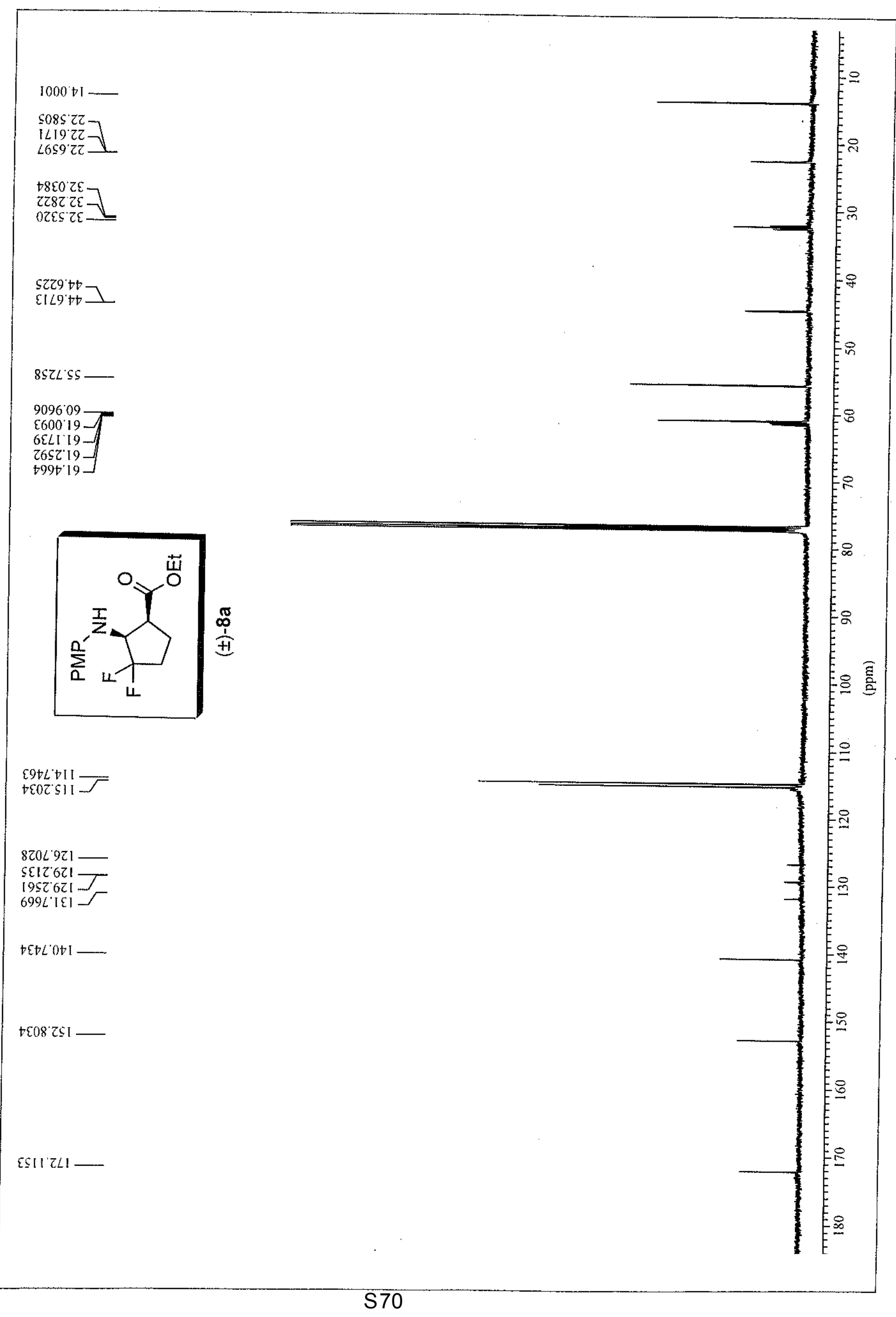




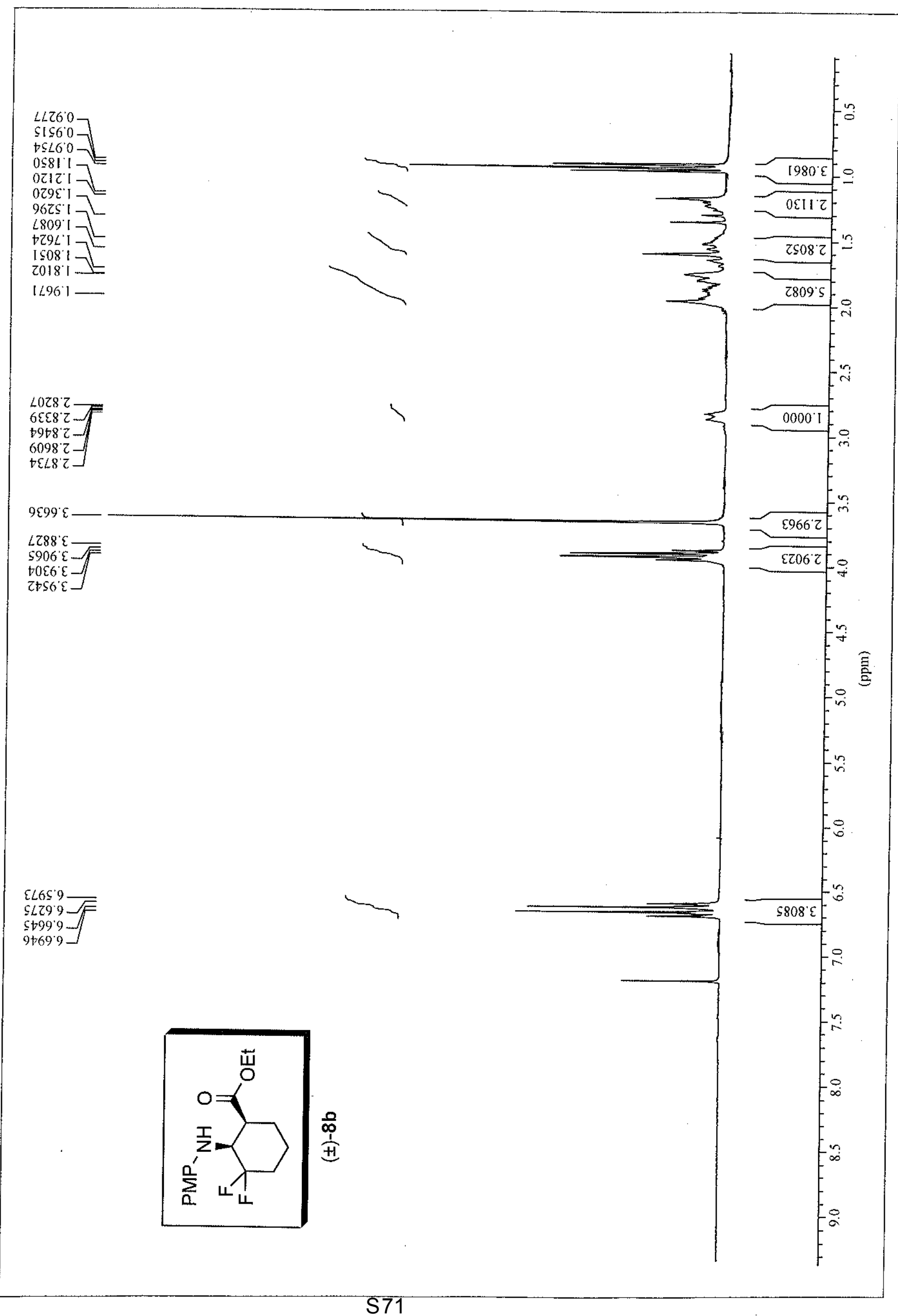




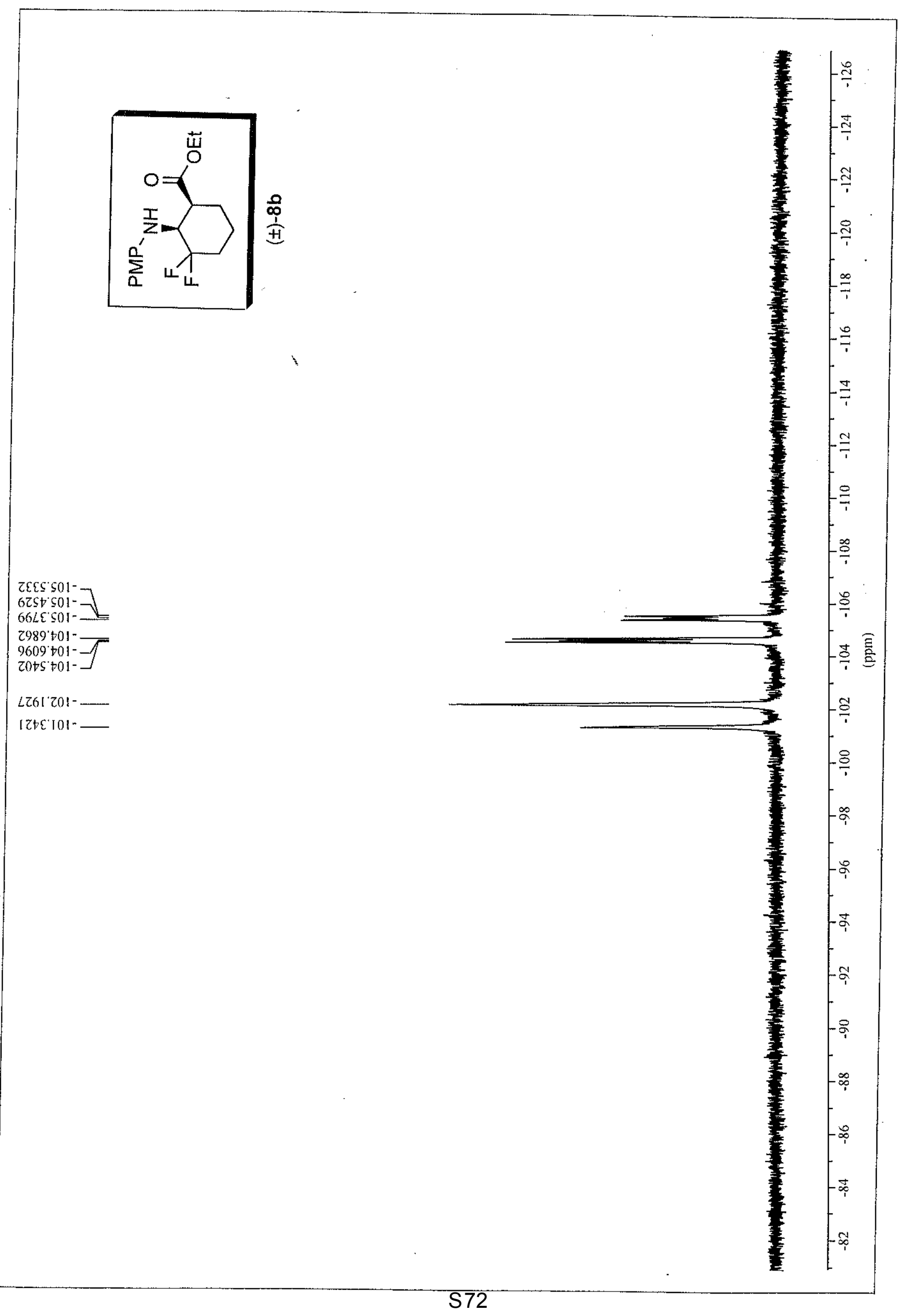




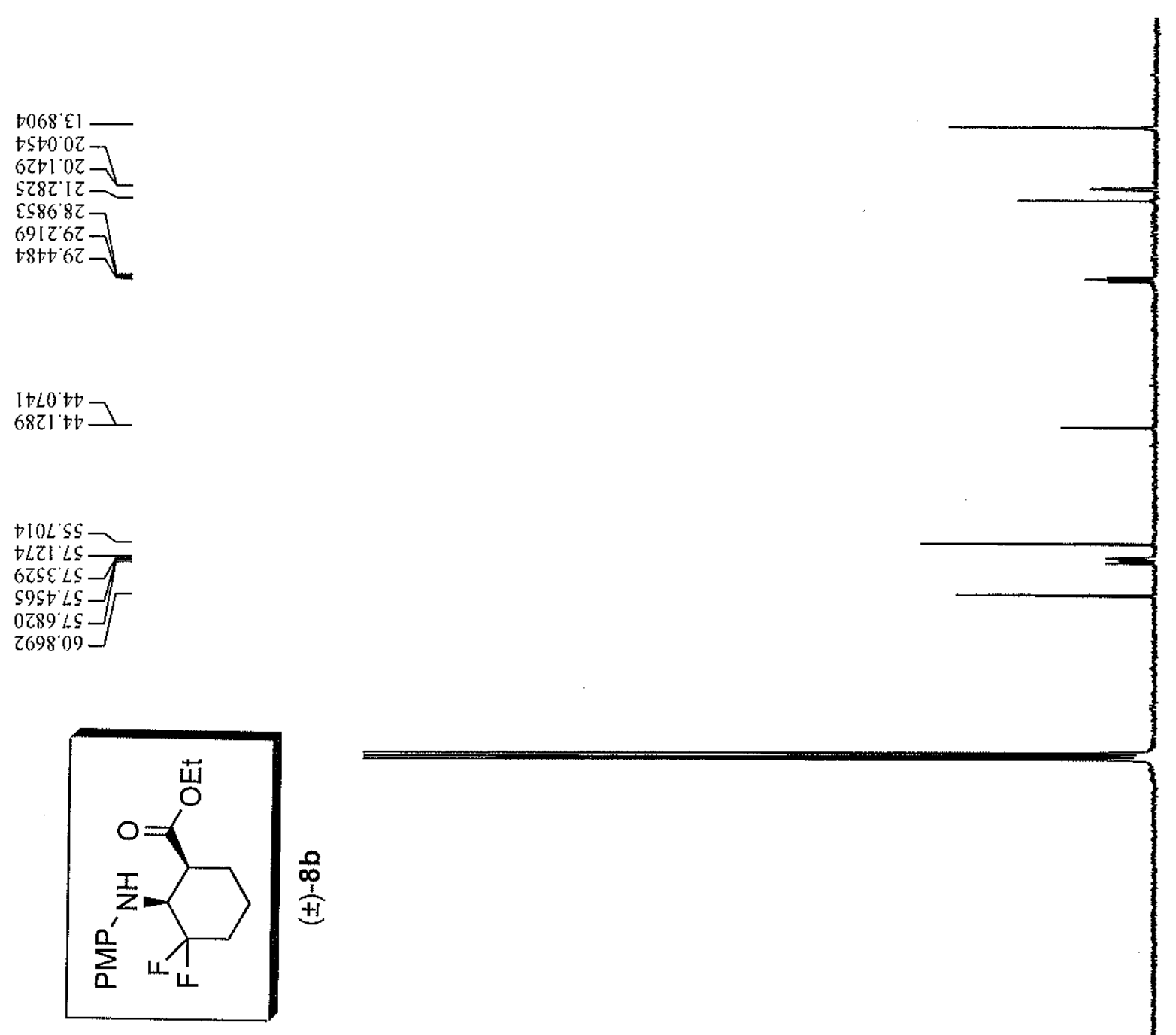

$1909+11-$
$\angle 119 S 11-$
$69 \angle 80 Z 1-$
296ZEZI-
†SLEEZI-
$\angle D 6 L \subseteq Z 1$

orEtリリ -
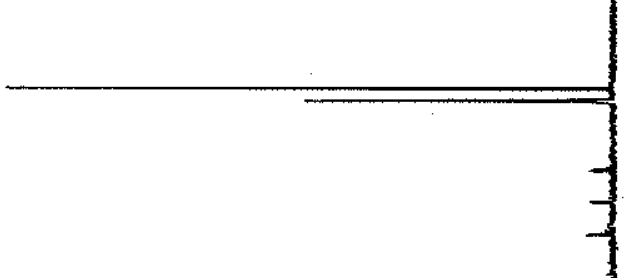
94260

$\checkmark 500^{\circ} \mathrm{t}$

ESZO I -

$828 \mathrm{LI}$

8702 '

$98 \mathrm{ZZ}: 1$

$0609 \cdot 17$

จㄴ.

90\$8: [ $]$

LOS $\mathrm{L}$ -

$2006:-$

$52002-$

$\varepsilon \varepsilon Z 0^{\circ} Z$ -

E62!' 2

SLEIZ

$9088^{\circ} \mathrm{Z}$

2 6682

$\varepsilon 806^{\circ} \mathrm{Z}$

$0+26 \% 2$

65E6"
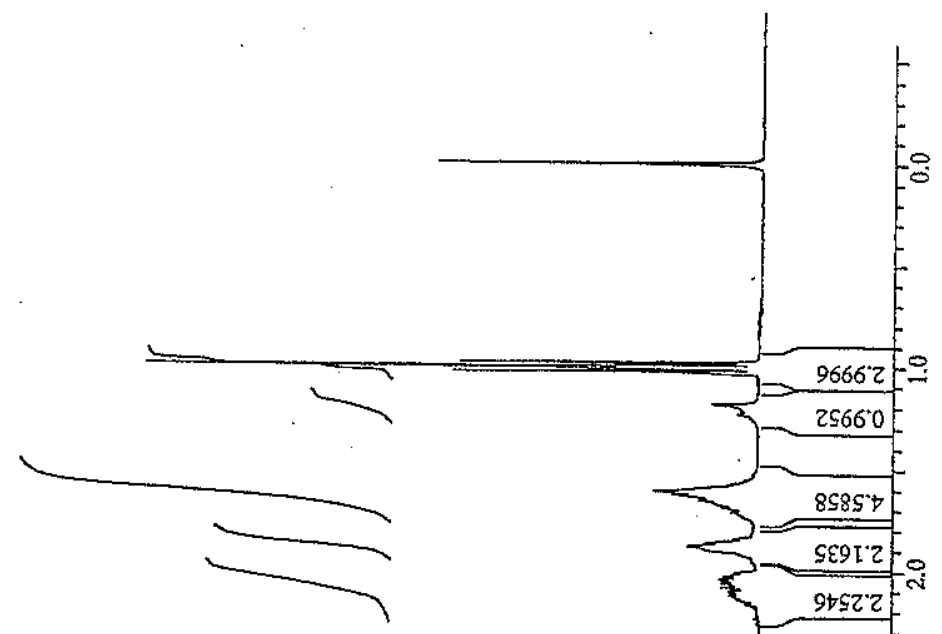

$9659^{\circ} \varepsilon$

$6 \forall \angle 8^{\circ} \varepsilon$ 一

เ $188^{\circ} \varepsilon-$

[868. $\varepsilon-$

$0 S 06^{\circ} \varepsilon-$

$6126 \%$

$88266^{\circ} \mathcal{E}$

$8576^{\circ} \varepsilon-$

$\left\llcorner 256^{\circ} \varepsilon-\right.$

$998 I^{\circ} t-$

sct [ $t \rightarrow$

$\angle L L T H-$

$8981^{\circ}-$

$6107 " t$

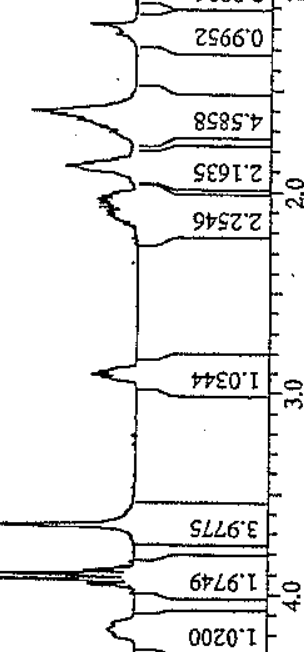

$8809^{\circ} 9$

I 6 E9 9

$8699^{\circ} 9$

$000 \angle 9$
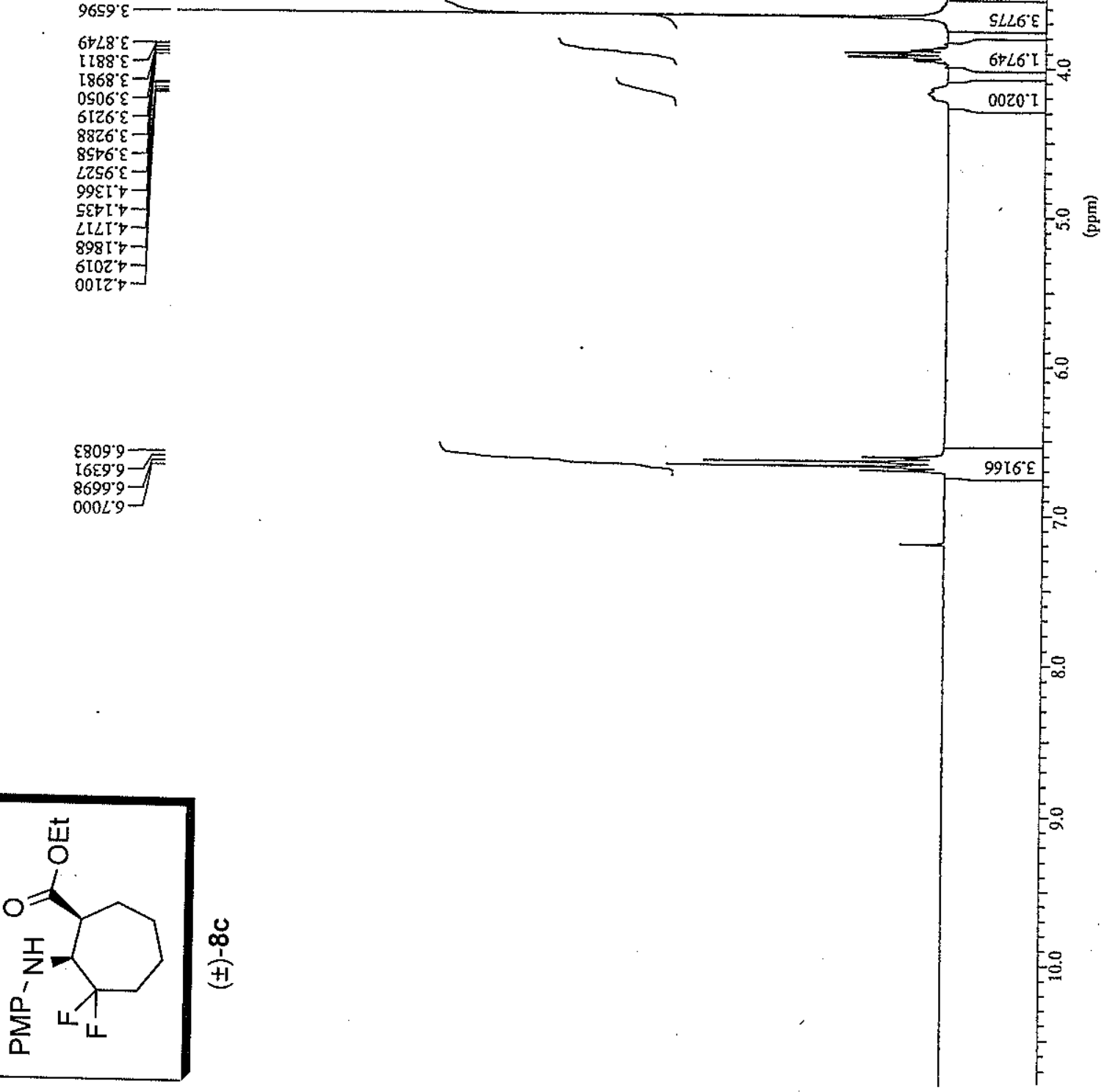

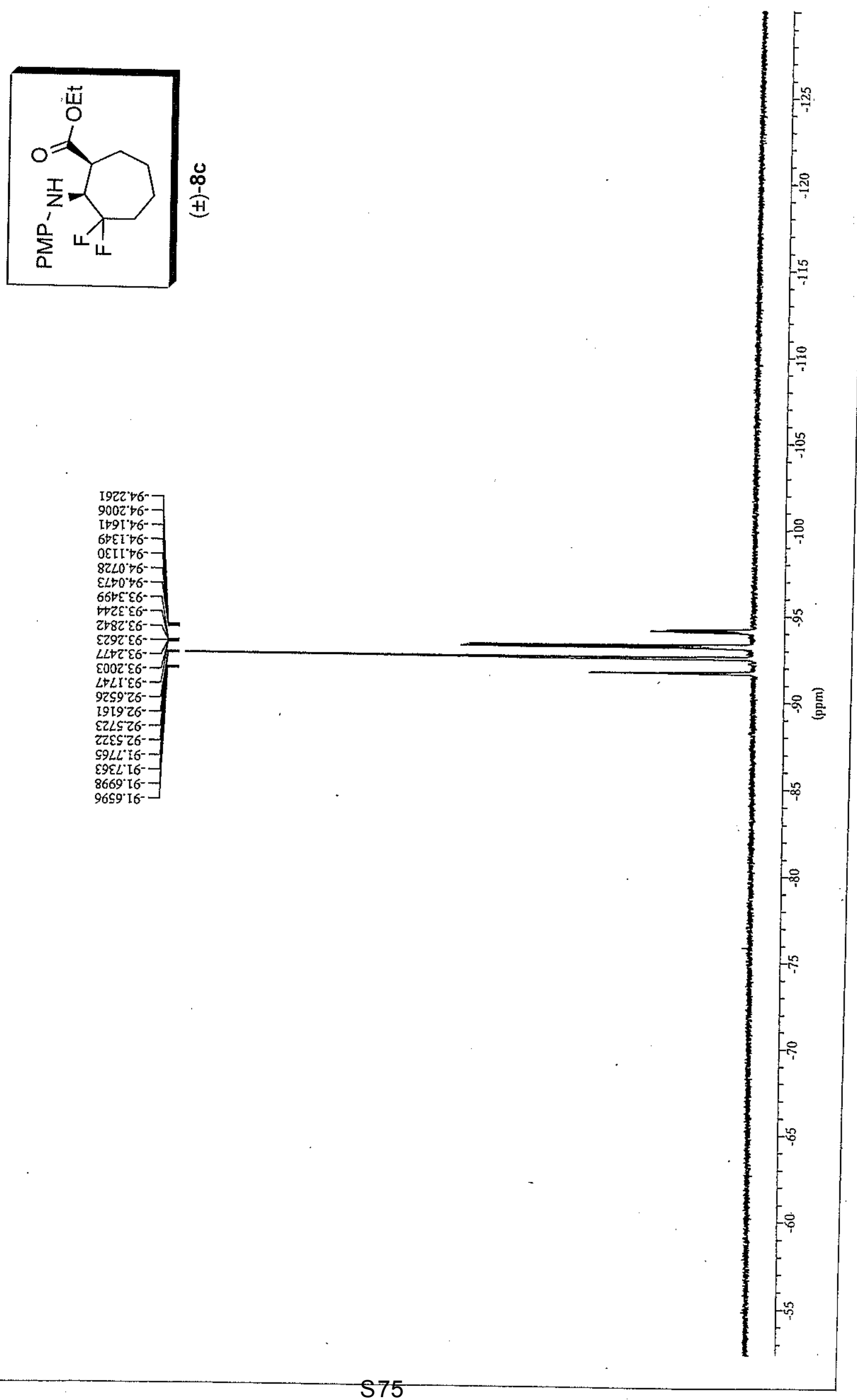


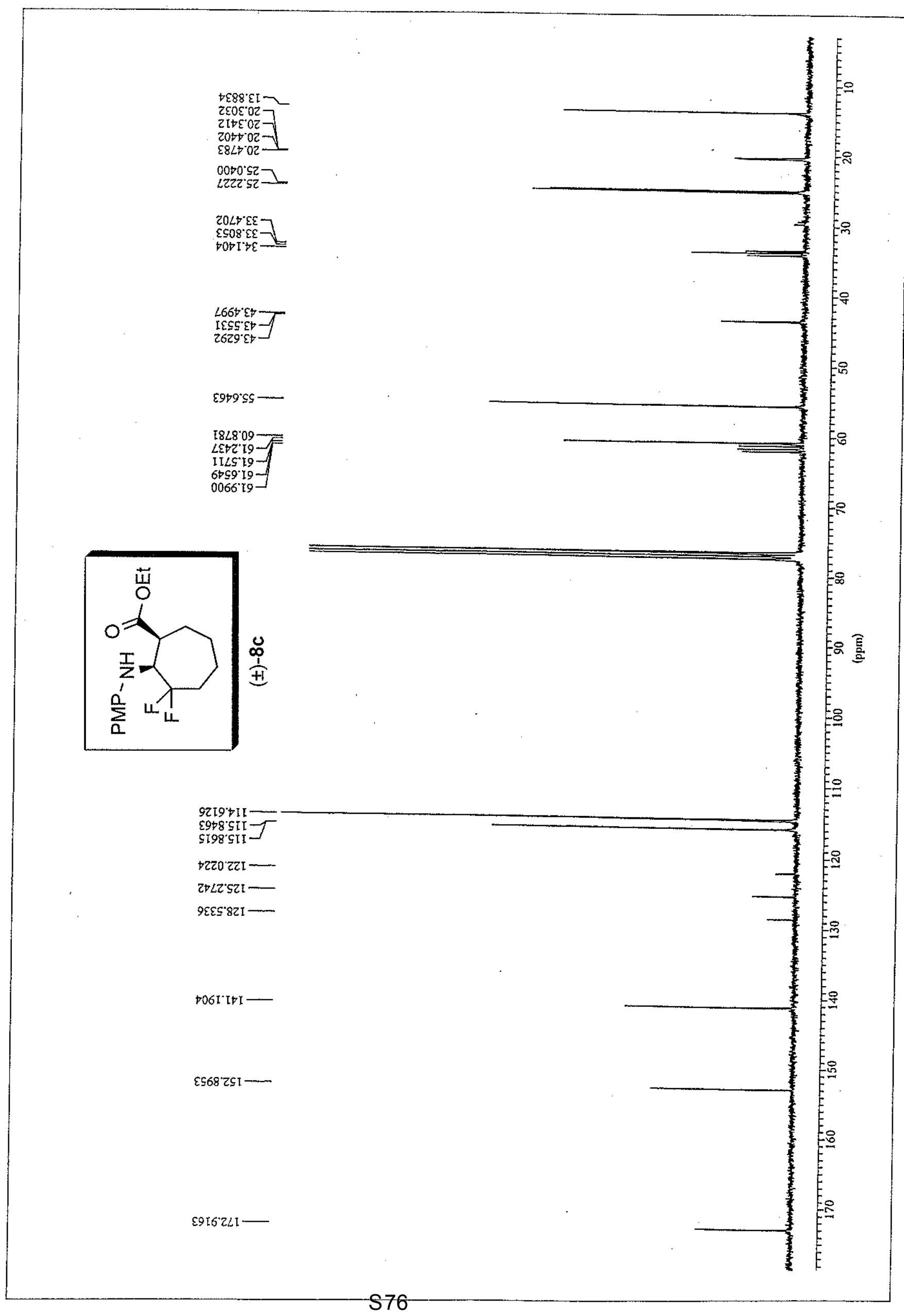




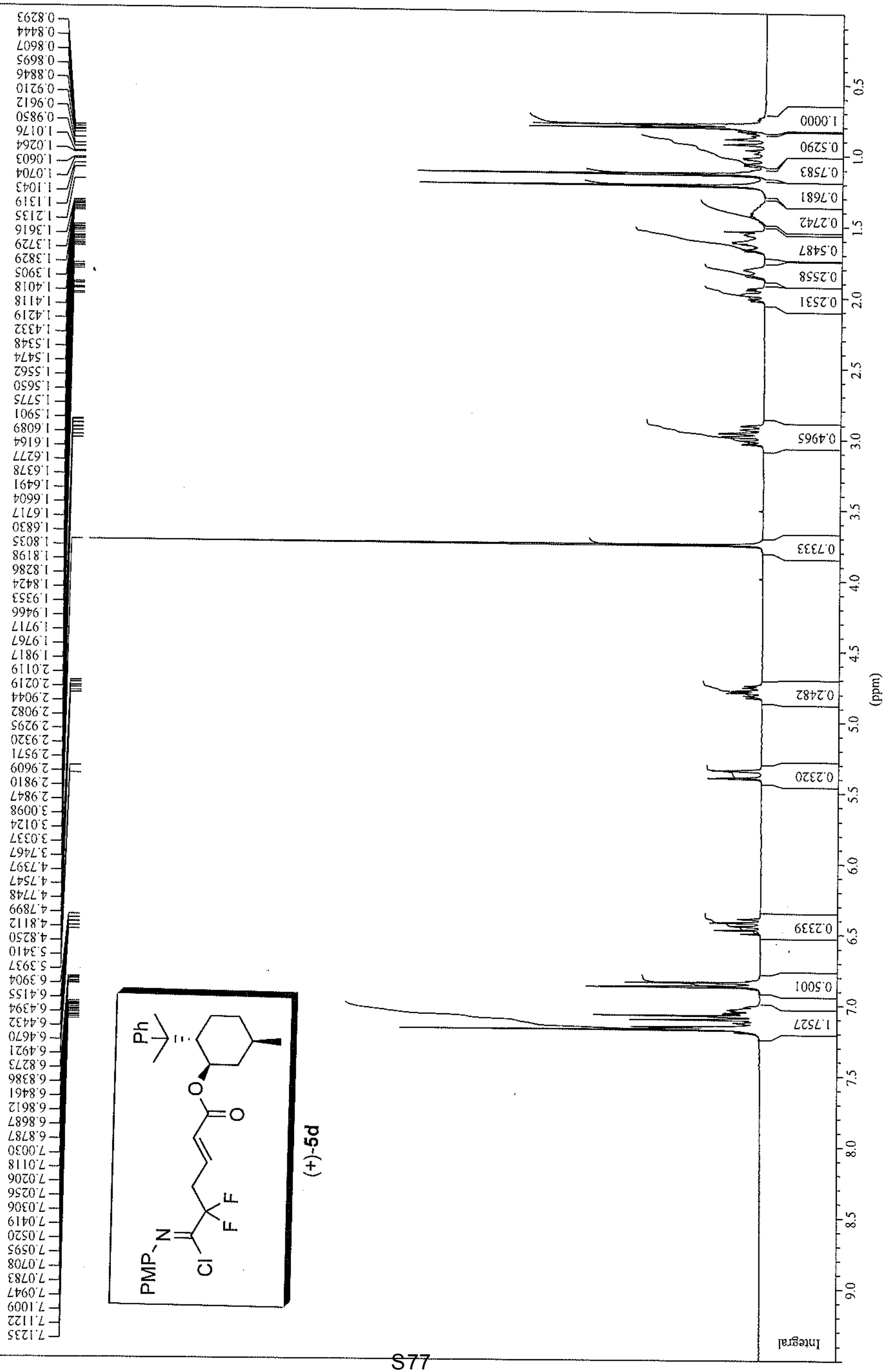


E208 $\angle 6^{-}$

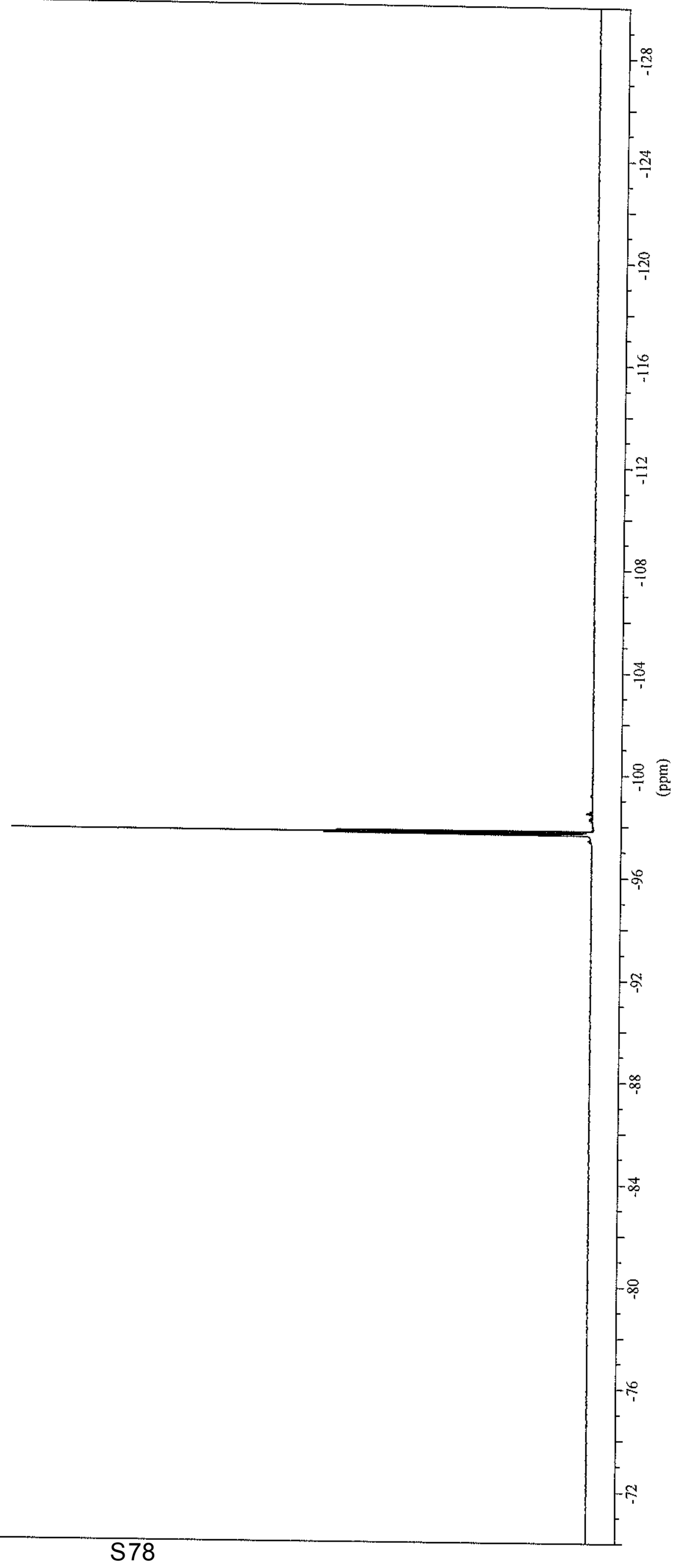


LLSLIZ

$6+0 L, t z$

st6ti9z

TSZ0 82

S9tZ'IE

$88 \mathrm{ZS}^{\prime} \rightarrow \mathrm{E}$

$866 \varepsilon^{\circ} \mathrm{LE}$

GtELLL $L E$

¿Z90' $8 \mathcal{E}$

DS8S $6 \varepsilon-$

SE09 It

LO6tOS

SSZt\$S -

$6980^{\circ} t L$

$2185^{\circ} 9 \mathrm{~L}$

S9Ot LL L
9SZ\&\&Il-

$\angle \nabla 60^{\circ} t 11-$

ItS† EZ|

ELI8.จZI-

coseszI -

SLOELZI

$\varepsilon+26: 2 Z 1]$

L669 $\varsigma \& 1$

L† I $\downarrow 8 \varepsilon \forall 9 \varepsilon 1-$

ร66t $9 \varepsilon 1-$

6L9S $9 E 1-$

L689 $9 \mathcal{E}^{\circ}[-$

OSSSISI

OZ8L8SI

$8899^{\circ}+91$

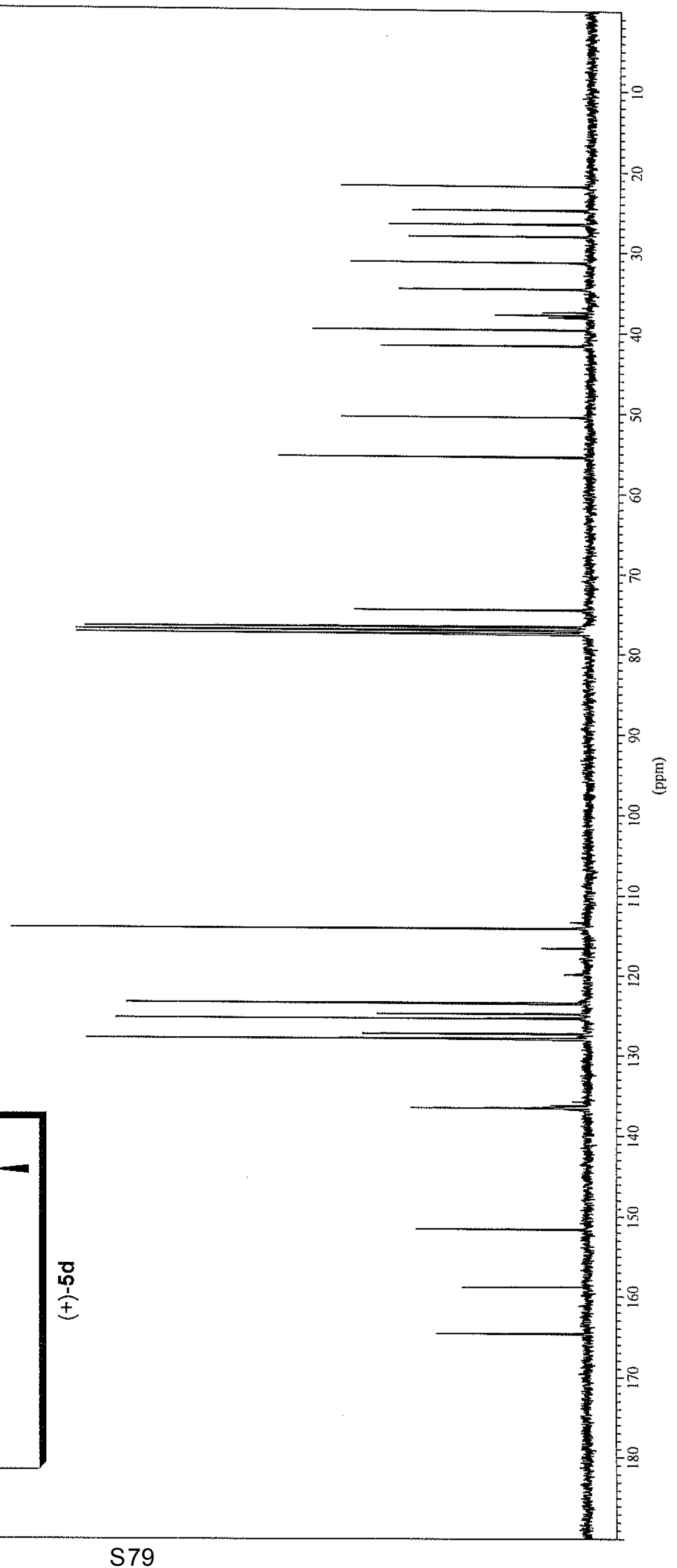


$96 \varepsilon E^{\prime}$ -

60s.'

609 \&

$789 E^{\circ}$ !

LGLE I

Ol $6 \varepsilon^{\circ}$

11001

DCiti

98 It l -

667t !

¿SZS I -

OESS I -

$08 S S^{\circ}$

8995

9SLS I-

$768 \mathrm{~S}^{\circ}$

61091

LSI9 I

ILE9

$5899^{\circ} 1-$

$86 \angle 9^{\circ} 1$ -

$8689^{\circ} 1$ -

EZOL:

DZI LI -

$6 t Z L 1-$

$S L E L !-$

EISLI

$109 L^{\circ} 1$

$10 L 21-$

LZ6LI-1]

EEट6। -

$8 \subseteq\left\{6^{\circ}\right]-$

$\angle 6 S 6^{\circ}:-$

$\angle 7966^{\circ}-$

$0[\angle 6] 1$.

$66661-$

ZIIOT-

LEZOZ

ISt0' $\mathrm{Z}$

$8550 \mathrm{Z}$

$\angle L L O Z$

$0060 \mathrm{z}-$

$0660 \%$

I 6012

此 $\mathrm{Z}$

oss! $z$

IE9! $Z$

0IDL $\varepsilon$

$8 \nabla S L ' \mathcal{E}$

9टIL口-

$L L Z L D+$

$060 \angle t D-$

$829 L \forall-$

$\bar{C}+8 L D$

$266 L ' t-$

6LE8' 9 -

$t S+88^{\circ}$

62589

\$098' 9 -

$0898^{\circ}-$

SS $\angle 8^{\circ}{ }^{\circ}-$

$8988^{\circ}-$

$00 t 0^{\circ} \mathrm{L}-$

EISOL

$8850 \mathrm{~L}-$

$9 \angle 90^{\circ} \mathrm{L}-$

$2080 \mathrm{~L}$

S $160^{\circ} \mathrm{L}-$

$9081 \mathrm{~L}$

$|\varepsilon 6|^{\circ} \mathrm{L}$

$6902 L$
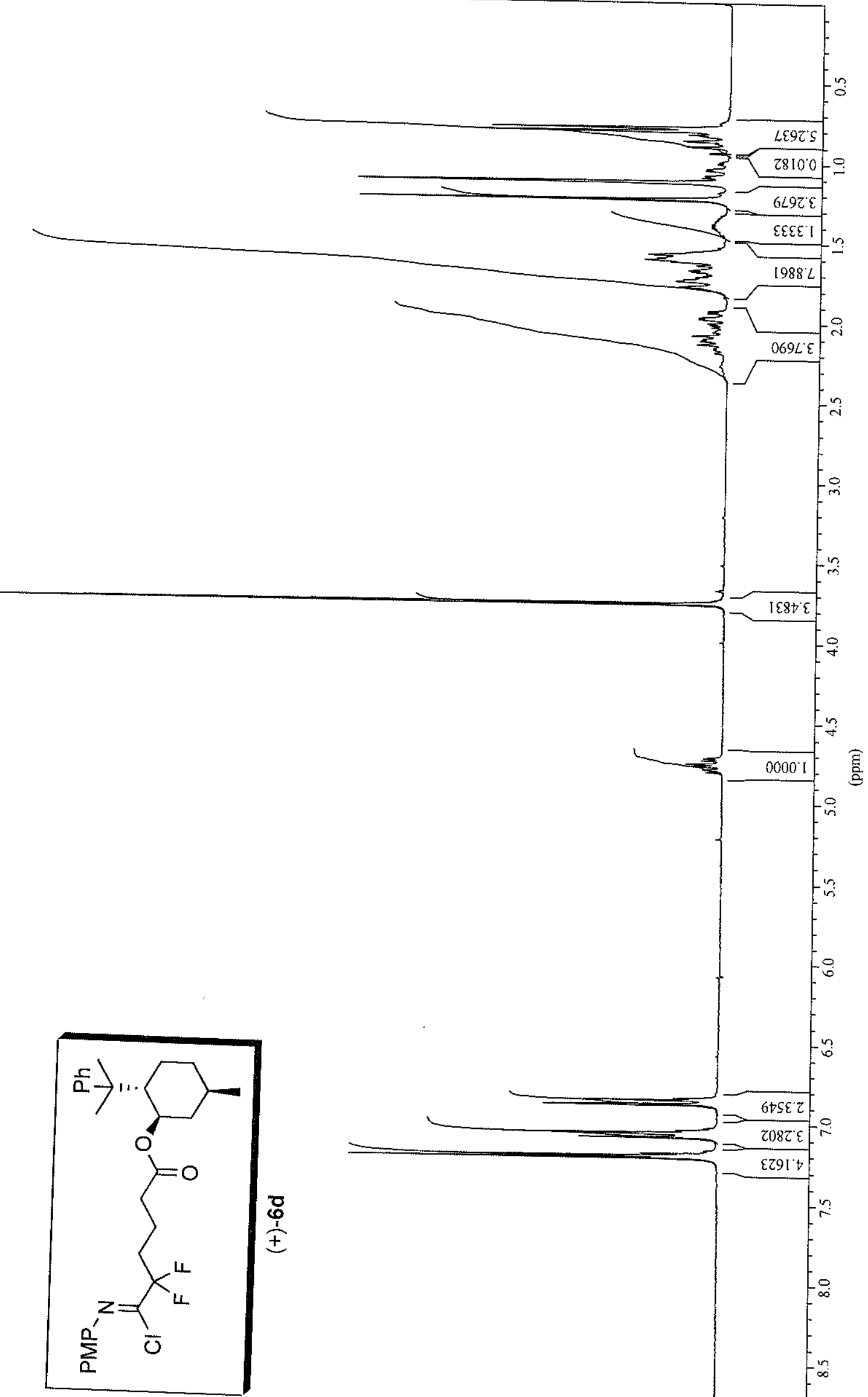

웅

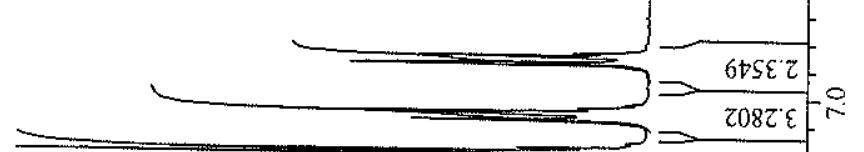

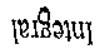


$\angle E 66^{\circ} 66^{\circ}-\bar{\Gamma}$
$2 \operatorname{soc}^{\circ} 66^{\circ}$

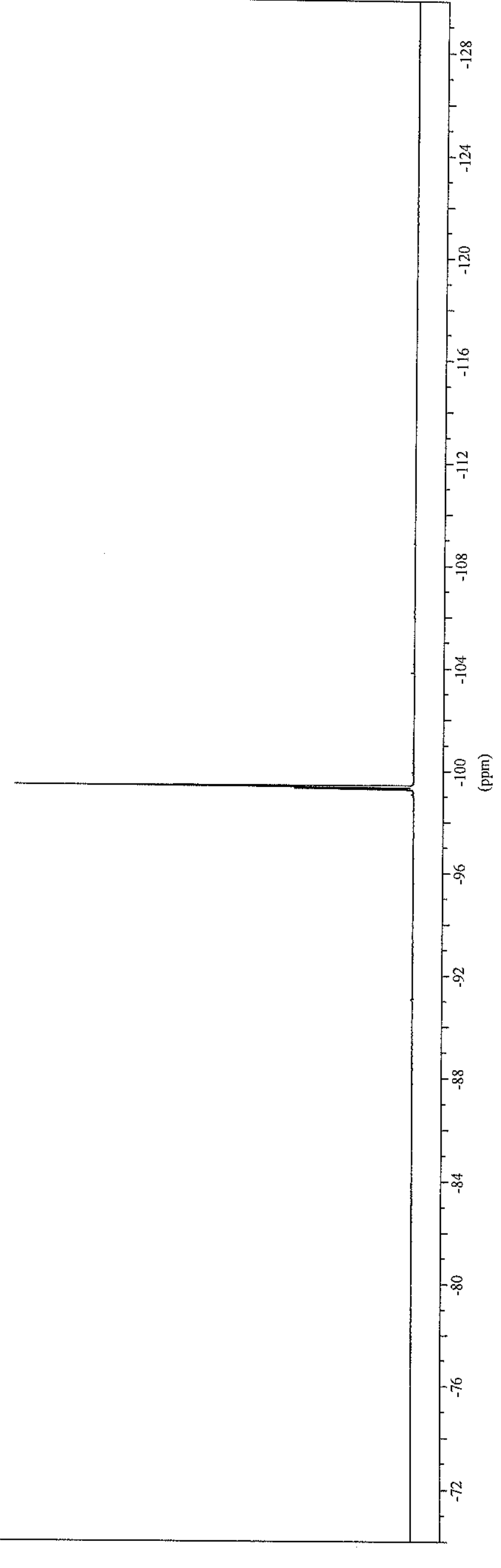




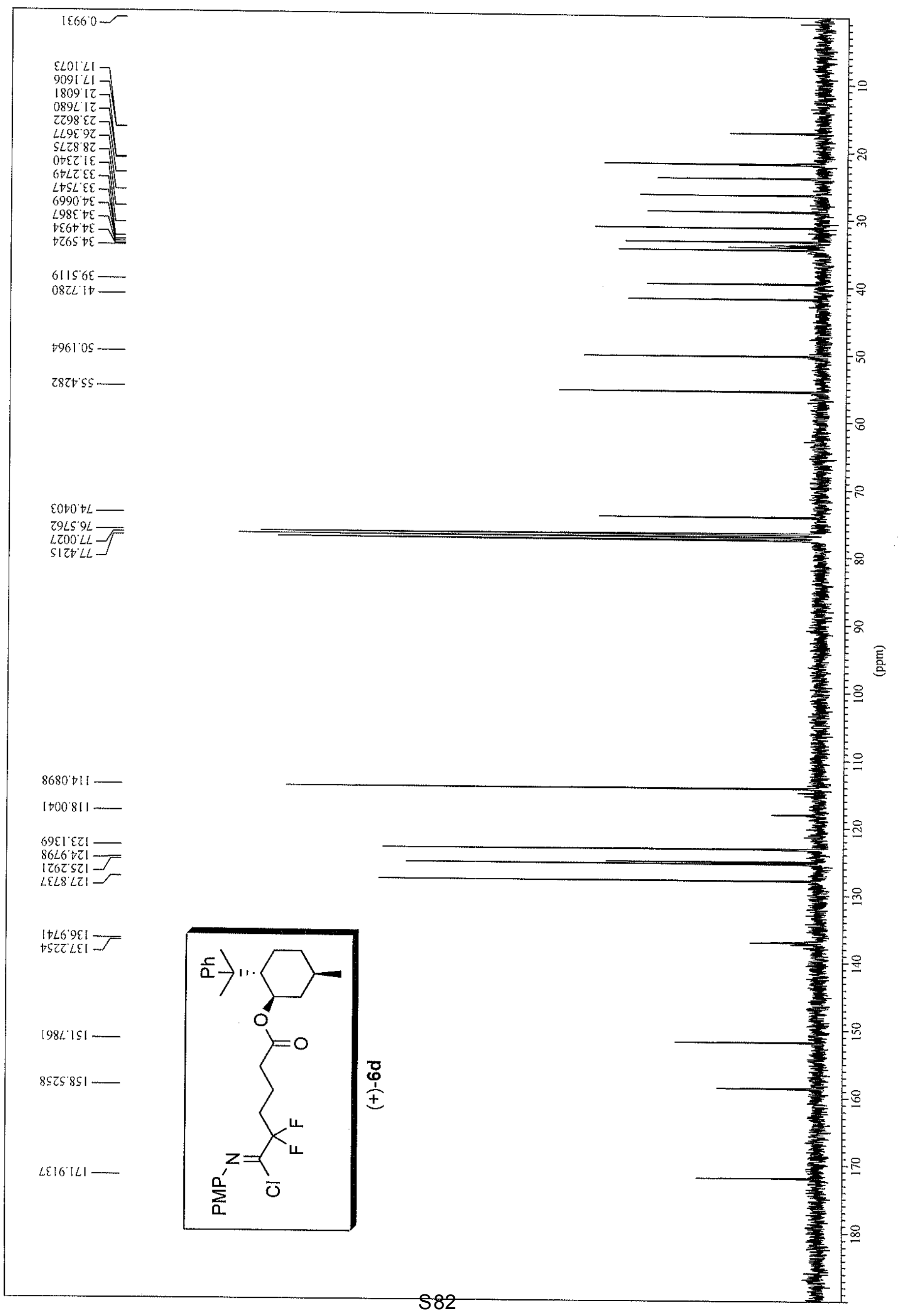




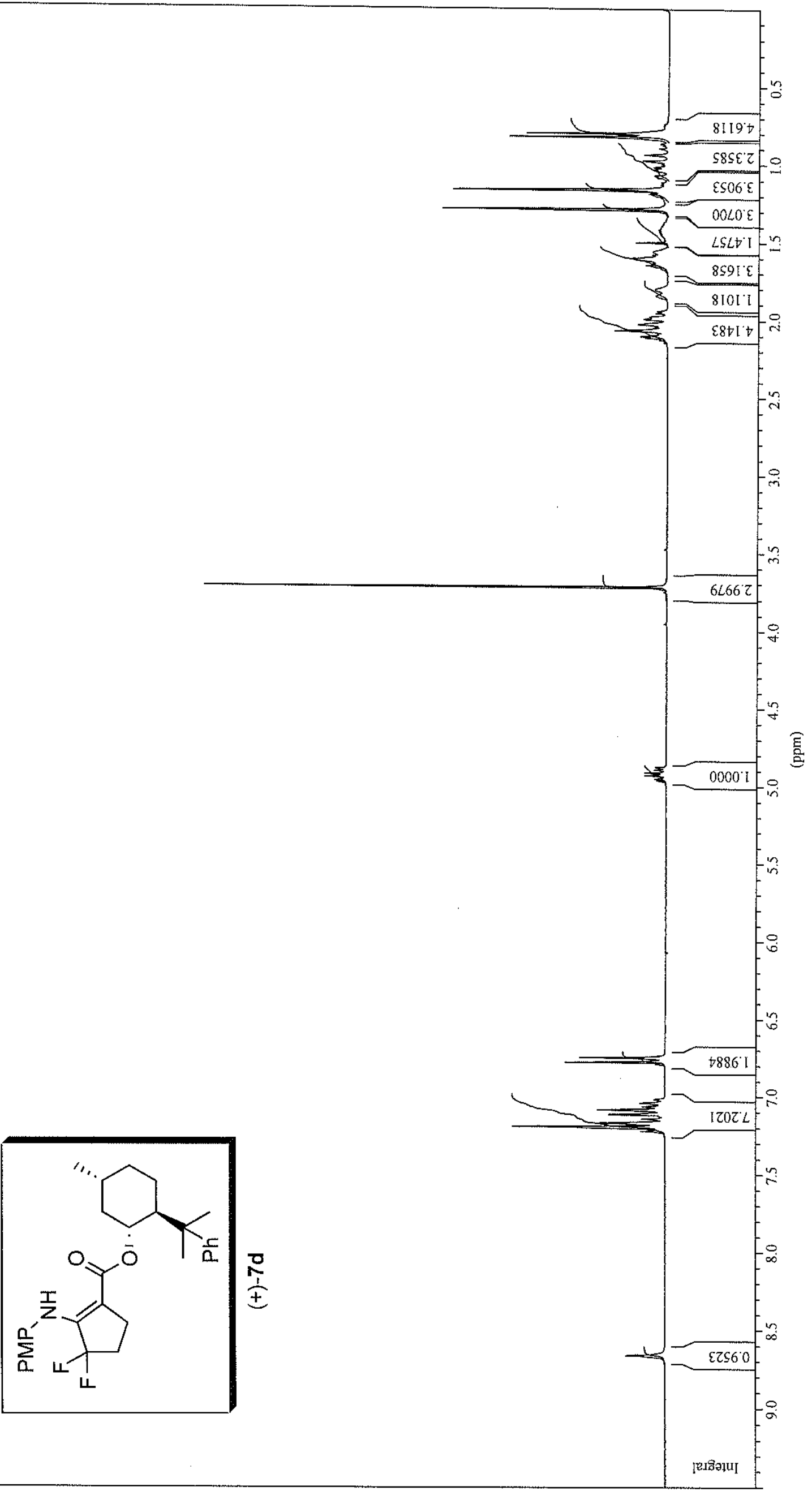




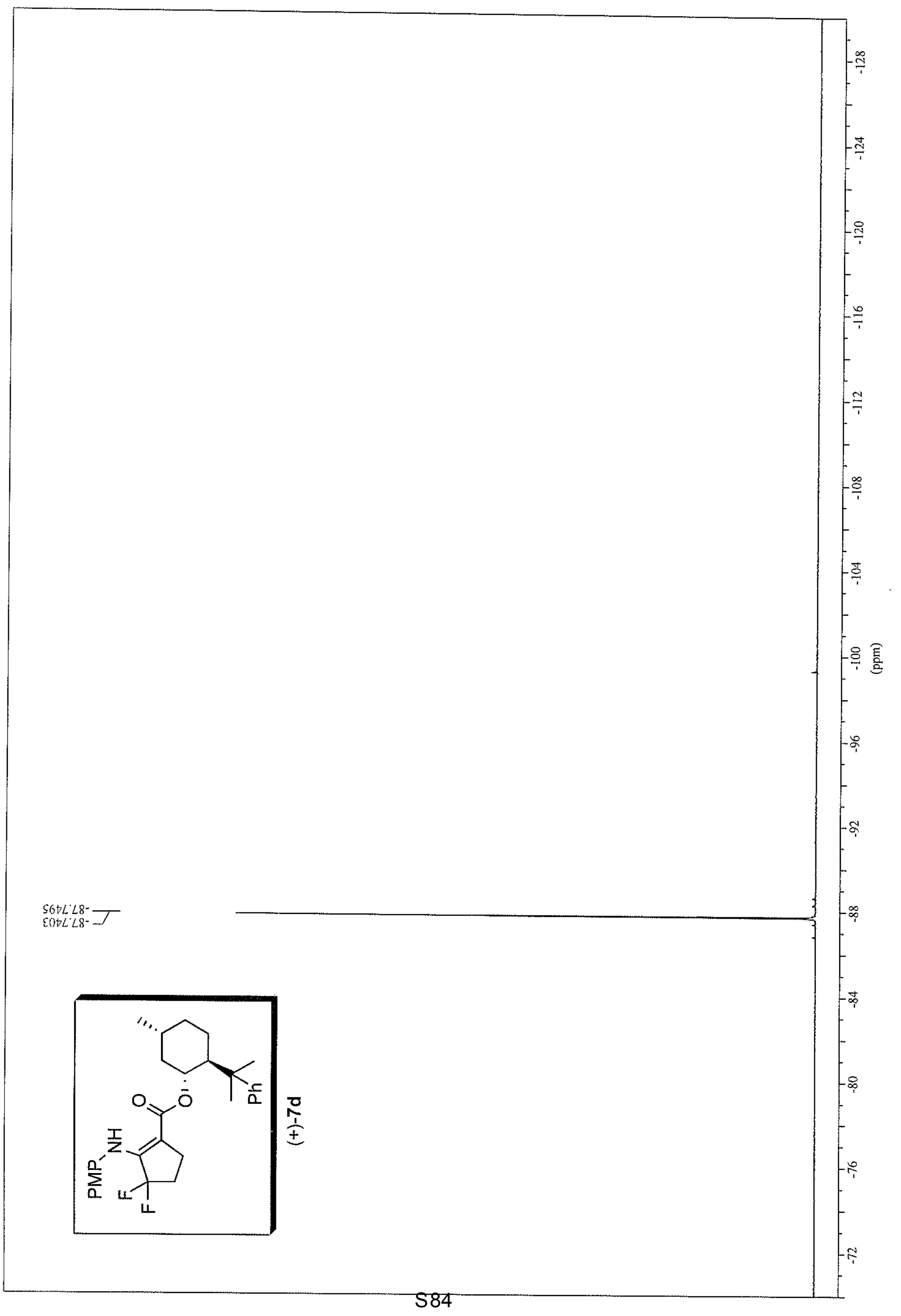


$\varepsilon 108: 12$

$\angle 166127$

$8962 \varsigma \mathrm{L}-$
$1869^{\circ} \mathrm{Z}-$

$8 Z E 8 \angle Z$

Z68) IE-

GlLS'IE

$97062 \varepsilon-7$
IZ£Z $£ \varepsilon-7$

GISSEE

$\varepsilon+\varepsilon \varsigma\llcorner\varepsilon$

SBSL $6 E$

tost $2 t$

L9zSOS -

10LE'SS

$908 \varepsilon \& L=$

$16 \angle S^{\circ} 9 L$

$9500 \mathrm{LL}$

$\forall t Z t+L L=$

$9918^{\circ} 101-$

$9 \$ 16^{\prime} 101-7$

$\angle 768^{\prime} \varepsilon 11-$

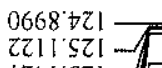

$\angle Z D I S 21-]$

$11<\varepsilon s z 1=$

00E9'szl

$0698^{\circ} \mathrm{LZ1}$ -

$6616.821-$

$\angle \forall \angle 8 \cdot 1 \varepsilon 1-$

$0 \leq Z \tau \tau \varepsilon 1-$

9829.811

6006801

s\$2 $601-5$

6E19 $\mid 5]$

$\$ 626951$

$8288991-$
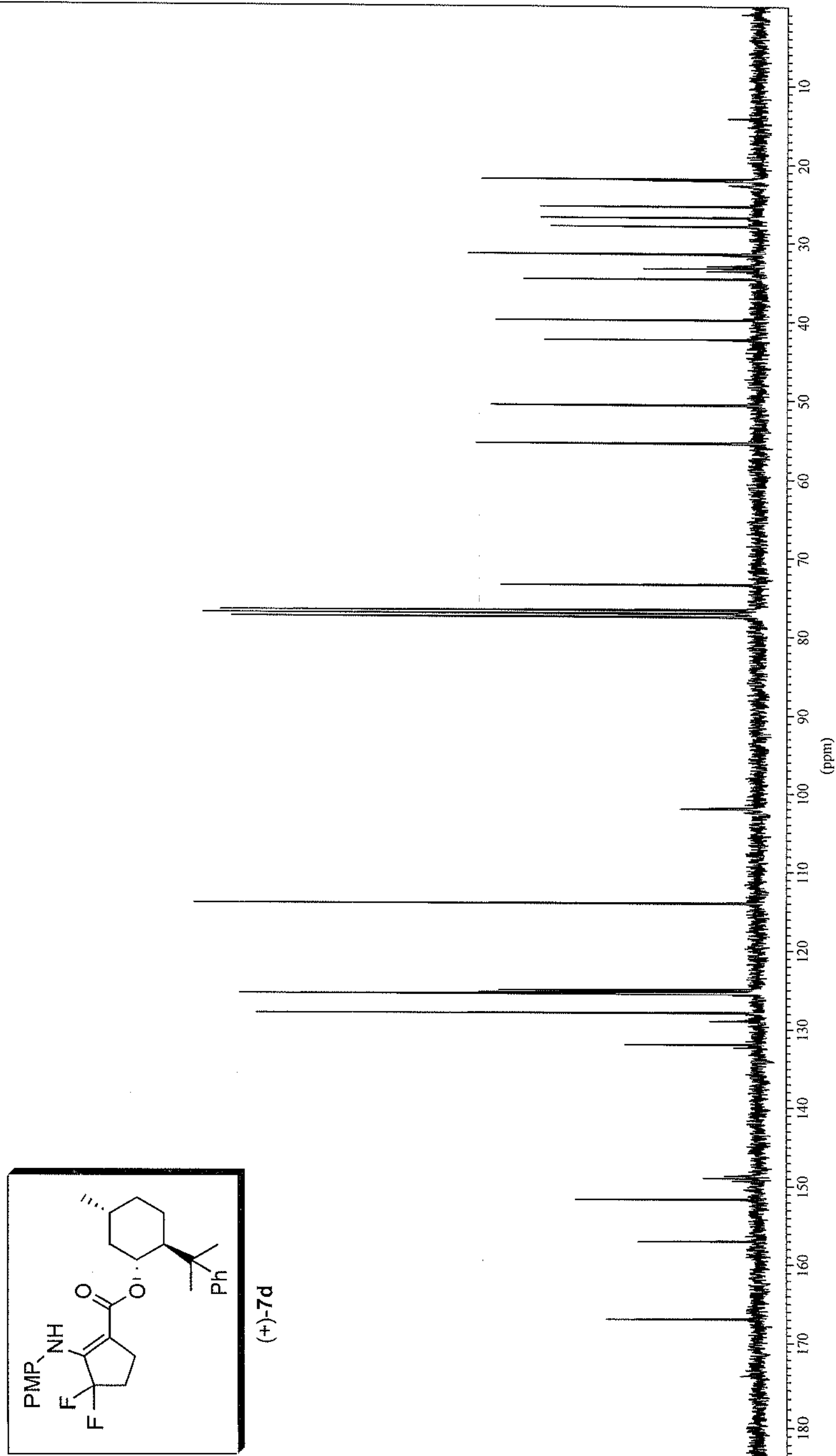
$896 \varepsilon 0$

$1605 \mathrm{I}$

LLSS

SEE9

$579^{\circ}$
$\nabla 5 \$ 9^{\circ}$

$1 \angle 99^{\circ}$

Z8L.

¿269

9ZZL'I

$09 z 8$

6LE

59981

¿EL8 I

$0206^{\circ}-$

$8 \mathcal{E} 16.1$

2996! -

E9L6 I

¿E66 I-

$6+002$

zs10z-

$+\subseteq \varepsilon O Z$

$\checkmark 6 S 0 ' z$

z+9l'z-

111zz-

$\forall \subseteq \varepsilon Z Z-$

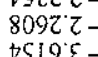

$\nabla S 19^{\circ} \varepsilon$

$\Delta \varepsilon+99^{\circ} \varepsilon-$

I $189^{\circ} \varepsilon-$

o $\varepsilon 9^{\circ} t-$

$0699^{\circ}-$

$\rightarrow \varepsilon 89{ }^{\circ} \rightarrow$

$t+0<' t-$

$261 L t-$

S9で 9

$29579=$

\$9t9. 9

0099 -

61299

$+6299$

$9+69^{\circ} 9-$

$\angle 10<9-$ E

$6 \varepsilon 129-$

$\triangle 290 \mathrm{~L}$

$\angle \varepsilon \angle O^{\circ} L$

EZ80 $\mathrm{L}$

$\angle \forall 60 L$

$0201^{2} L$

EIIIL-

$0691 ' \mathrm{~L}-$

E161' $L-$

$\angle L 61 \%$

ZEIZ $L-$

$80+Z^{\circ} L$

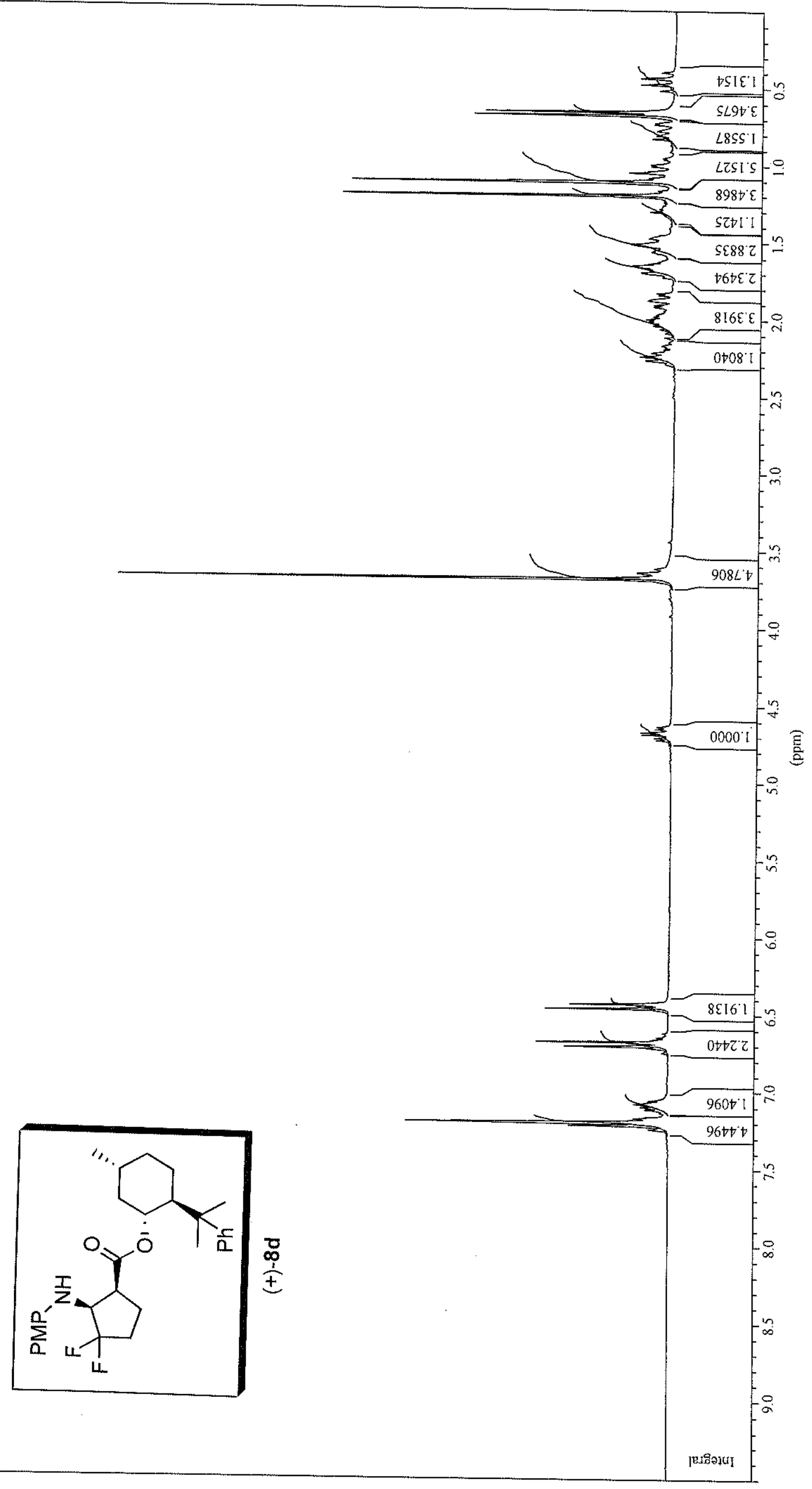


$\angle S \& 9^{\circ} 60 \mathrm{I}^{-}$

$1019601-$

$2885.601-$

$8815601-$

$2528.801^{-}$

LLLL'80I"-

ZZSL $80 \mathrm{i}^{\circ}$

$\$ 80 L 801 \%$

$9 \triangleright \varepsilon 266-$

$0861^{\circ} 66^{-}-7$

$19 \angle 166^{\circ}-$

$\varepsilon \varepsilon \nabla I^{\circ} 66^{-}-$

t। $2 l^{\circ} 66^{\circ}-$

$6+80^{\prime} 66^{-}$

tozt $86^{-}-$

LS9E $86^{-}$

8ZЕ $86^{-}$

601E $86^{-}-$

$\rightarrow \forall 286^{-}$

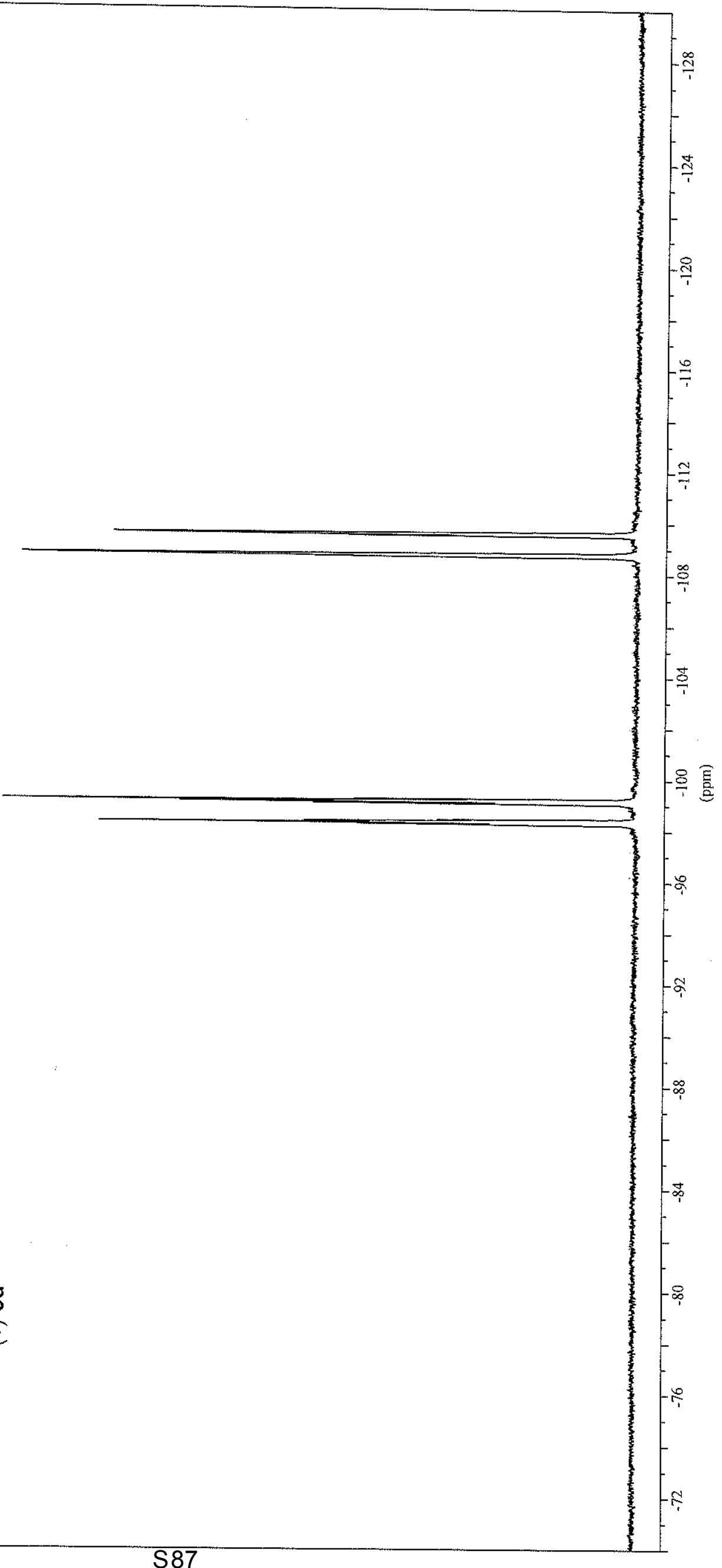


6L6S Iz

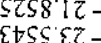

sะz 92

$28568 \mathrm{Z}$

$+960 \mathrm{LE}$
$8 \mathrm{~L} 6 \mathrm{C} / \mathrm{L}$

LESSIE

0I16.18

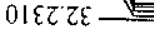

$\varepsilon 69 \varepsilon^{\circ} \triangleright$

6Stto $6 \varepsilon$

th117 -

ctocto

ISSE $t+\triangle$

$\$ 9800 \varsigma-$

S99LSS

[2!E.09-

$\$ 88 \subseteq 09$

$761<09$

$\$ 88609$

$Z \angle 88^{\circ} \forall L$

$8185^{\circ} 9 L$

$9 \varepsilon 00 \mathrm{LL}$

ELOZLLL

$\subseteq \subseteq Z+L L-$

$\tau \forall \varepsilon 9+11$

$0918+11=$

tZSZSII-

90ss sil]

$2866^{\circ} \mathrm{ZZ1}$

Z8!๕ऽZ1

ELSS SZT -

LEzo'gri -

$8 E 8 L L Z 1$

E695 621 .

SLZD 6 ZI

$0<\angle L Z E 1-$

DELLO $0+1$

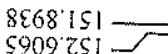

$81811 / 21=$
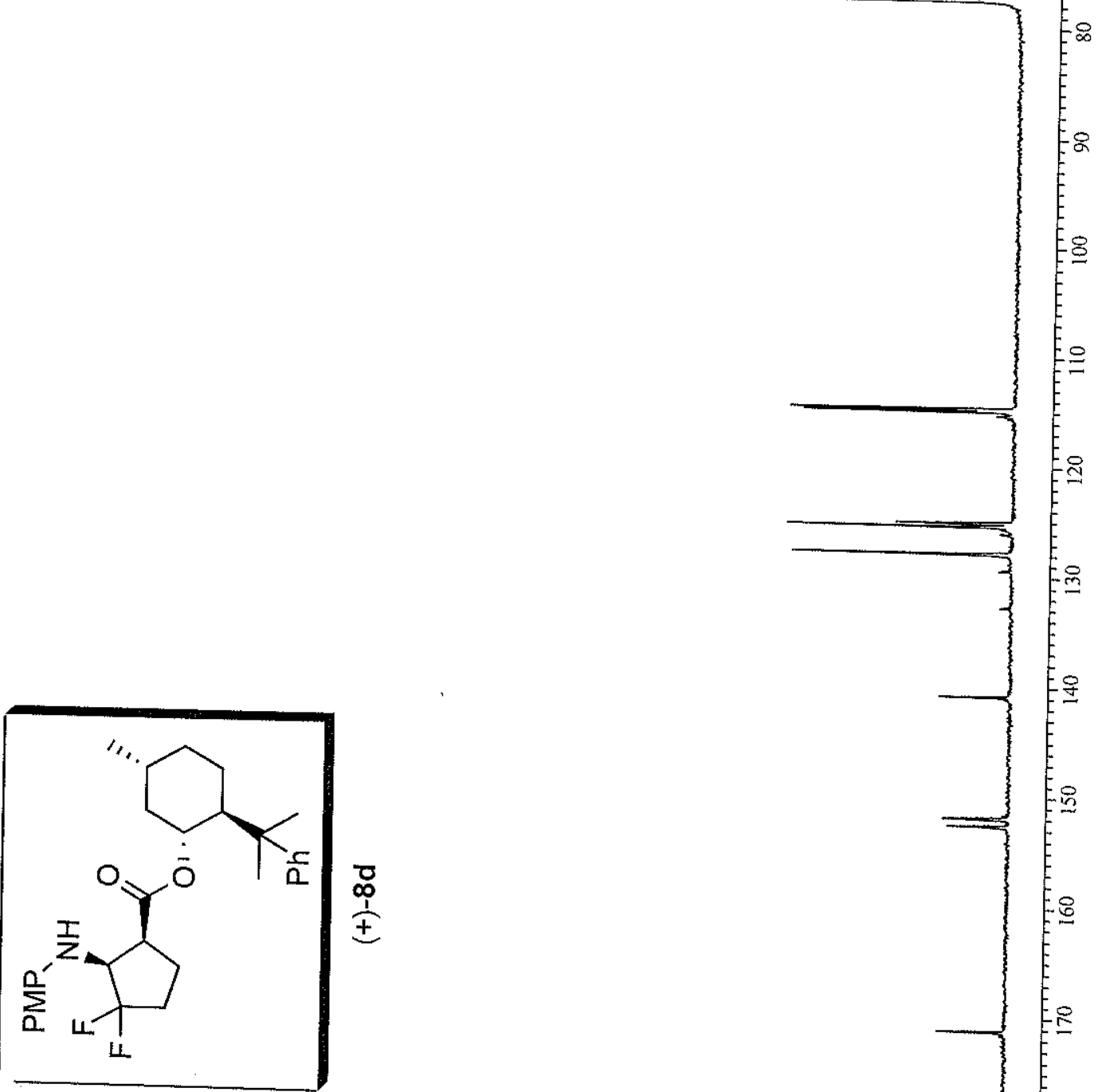
12590

$\tau \nabla \angle 9^{\circ} 0$

$\triangle 6 \& L^{\prime} O-$

6OSLO-

$6508^{\circ} 0$

$0898^{\circ}=$

$670607=$

$0 S 760$

$£ \$ 960$

686600

$29001-$

$\varepsilon 600 \mathrm{I}-\mathrm{C}$

Eะ80' -

SLI I

†9LII

zह6 I' I -

$\varepsilon 00 \tau 1$

6EZZ।

I $\angle S Z I 1$
$\angle \angle 6 C^{\circ}$

c8:

68 it.

0L951

t285

2119

0029

SLE9

$1+59^{\circ}$

$1 \angle 99:$

$\rightarrow$ 18.

IS[6.

$0 \angle 26^{\circ}$

S0S6:

82961

$6+86^{\circ} 1$

ह166

E1661-

$\varepsilon 100^{\circ} z-$

$\angle 0 \varepsilon 0^{\circ} \mathrm{Z}$

$1+502-$

(280?-

бटा1

$99 \varepsilon 1 \tau-$

\&SSt $\tau$

6LLTZ

$\cos 2-1$

$8 \operatorname{ctS}^{2} \mathrm{C}-$
$+\cos \mathrm{C}-1$

$t c 89 \varepsilon-$

$78 \nabla L \mathcal{E}-$

S19LE

$\varepsilon 18 L \varepsilon-$

乙L08 $\varepsilon-$

乙

$0869 \circ-$

tol L't

$8 \varepsilon E L^{\prime} \downarrow-$

$08 \square L \Rightarrow-$

$\forall 69 L^{\circ} \circ-$

$8 \varepsilon 8 L \mathrm{t}$

65299
$\square \varepsilon 899-$

$08+9^{\circ} 9$ -

$09599-$

$\angle 5 O L ' 9-$

St $L$ L 9 -

EZLL' 9

$\angle O E L 9-$

80019

$8010^{\circ} \mathrm{L}-$

$\$ 610 \mathrm{~L}-$

toE $0 \mathrm{~L}$

$20+0 \mathrm{~L}$

9I $50 \mathrm{~L}$

$\$ 850 \mathrm{~L}$

$1060 \%$

七 $500^{\circ} \mathrm{L}$

$8111^{\circ} \mathrm{L}$

$1611 \%$

टEEI $L$

SGS I $L-$

$\angle 061 \%$

$0002:$

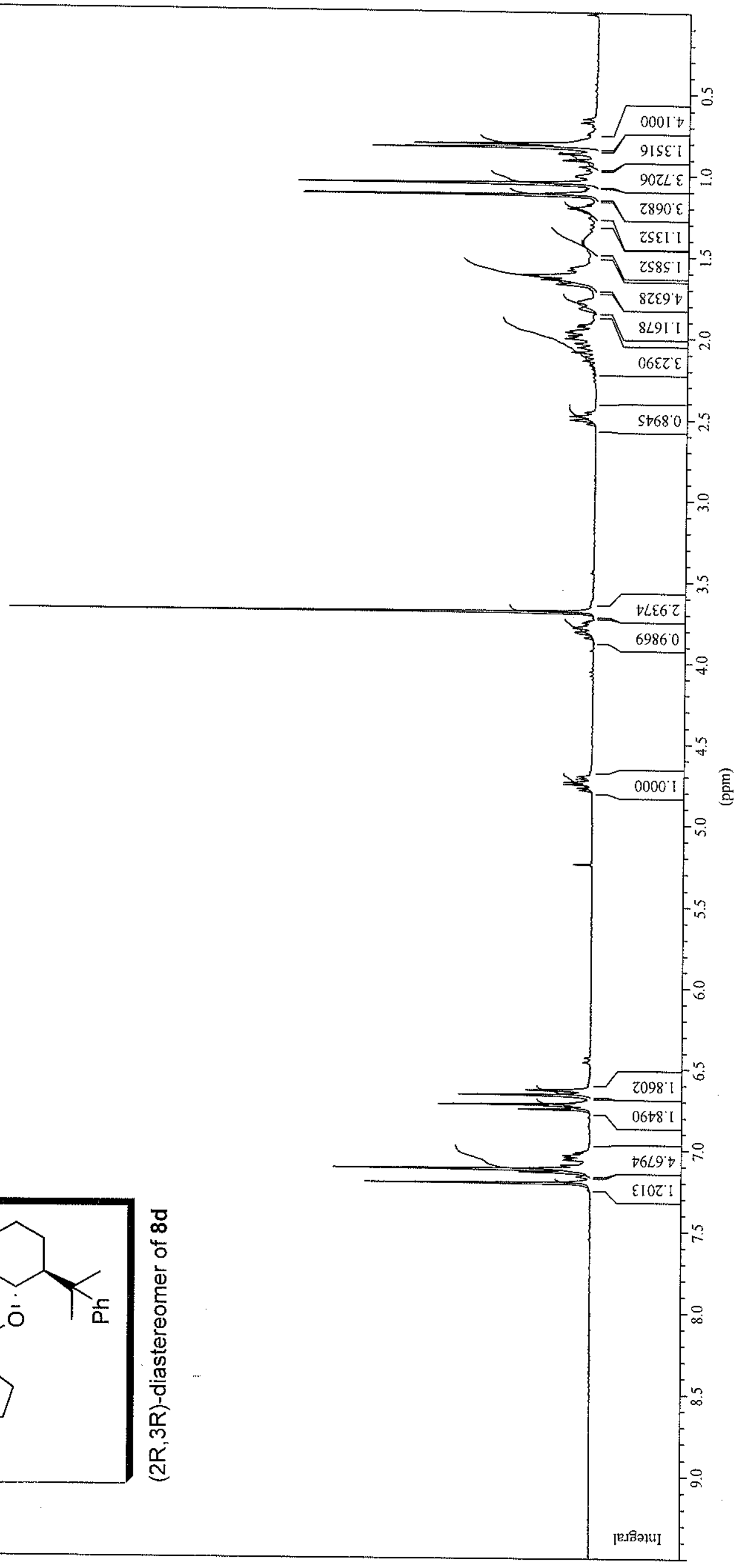


$\mathrm{SES8} \mathrm{SOI}^{-}$

6LZ8 $\mathrm{SOI}^{-}-$
$\mathrm{t} 20 \mathrm{~S}^{\circ} \mathrm{SOI}^{-}$

$\rightarrow 680 \mathrm{SOl}^{-}$

$8 \varepsilon 10 \mathrm{SOI}^{-}$

E886 tol-

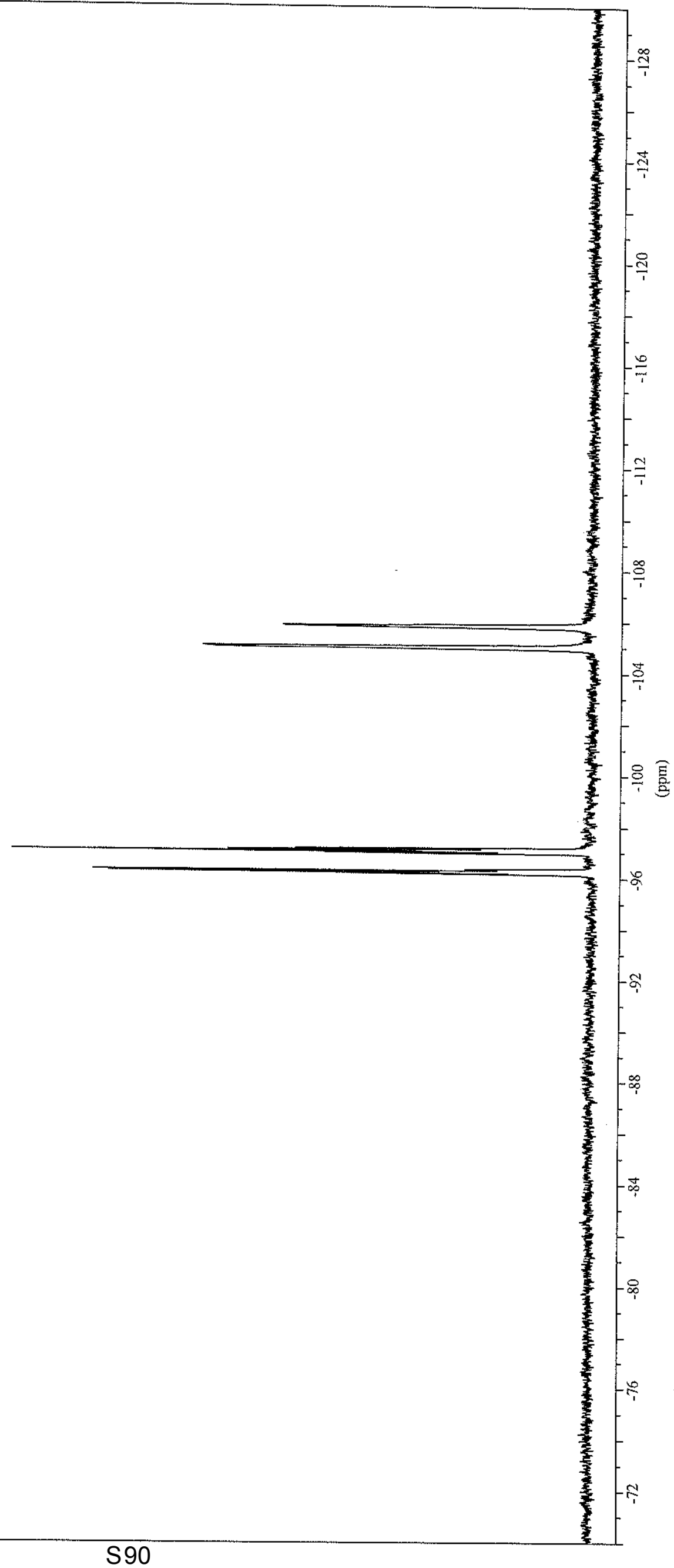

$69 t I^{\prime} L 6^{-}$

$2901.26-$

tISO $\left.26^{-}\right]$

0ह66 $96^{-}-$

$982896^{-}-$

I $262^{\circ} 96^{\circ-}$

$8 \varepsilon \angle \tau^{\circ} 96^{-}-$

LEEZ $96^{-}-$

68L196-]

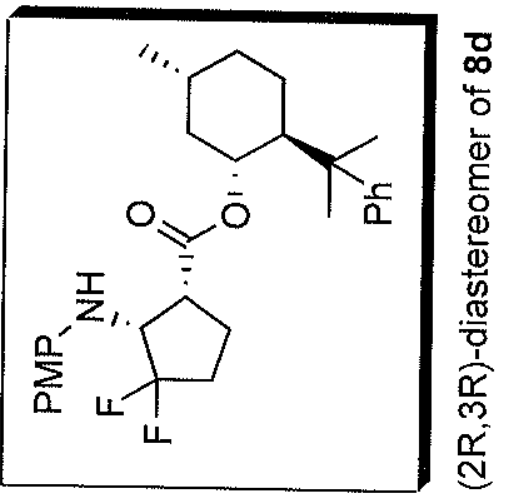


$860 L 12-7$
$915927-$

Llitoz

602592

$9500.8 z+1$

ऽ90E' [E]

6Z8S IE]

DL66.7E]

G659.2E

(IS)

OSzS $6 \mathrm{E}$

6997 it

$2 S+8^{\circ} \mathrm{C}$

6L16 $\mathrm{C} D$

o\&zน $0 \varsigma$

SOELSS

$6+8809$

S81119-

9L2 $19-$

EIIS'19...

$608 \mathcal{E}^{\circ} \mathrm{SL}$ -

$018 \varsigma^{\circ} 9 L-$

$8200 \mathrm{LL}$

$\$ 902 L L-$

$9 \nabla Z+D L L$

$\underset{\text { siLLt) }}{\text { stotsl }-}$

$6110 \mathrm{SZI}$

zocest -

$6 I \varepsilon L S Z I-\pi$

OZ68 $\angle 21-$

$5269^{\circ} 011$

$8829151=$

9968 Zs -

$\$ \subseteq 6 S^{\circ} 1 \angle 1$

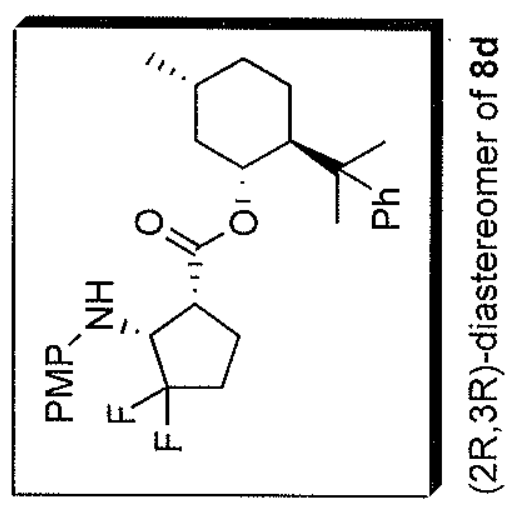




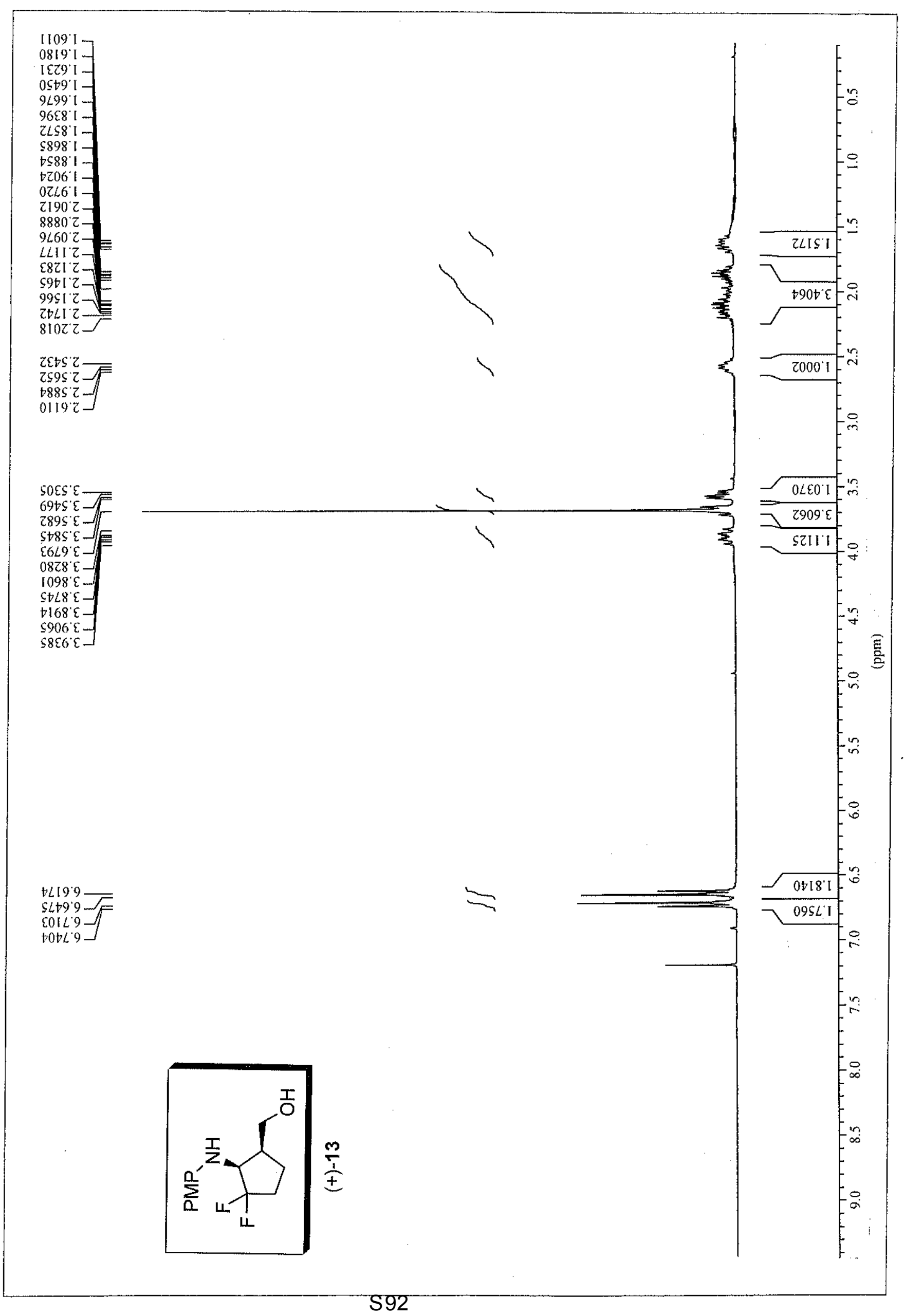




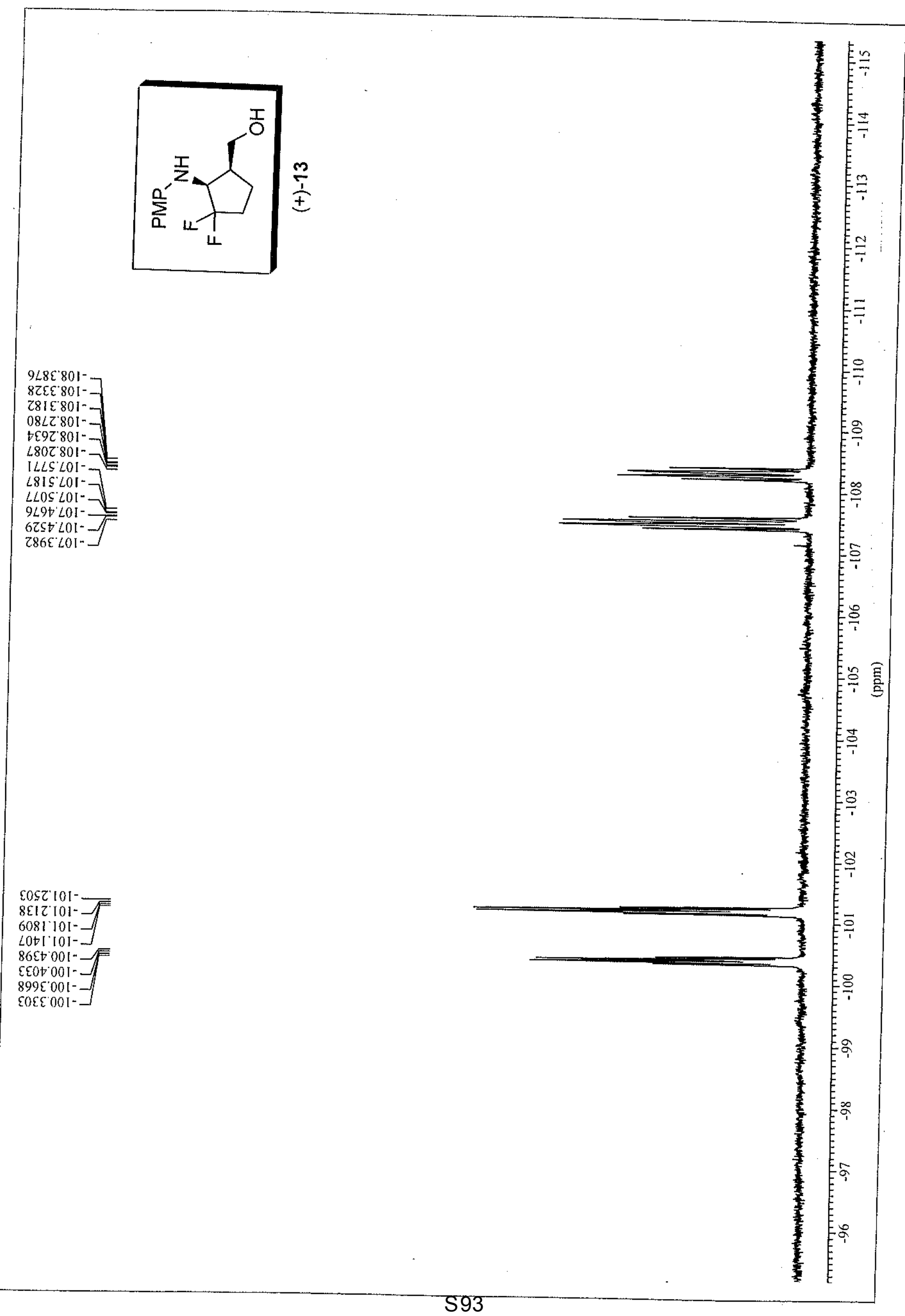




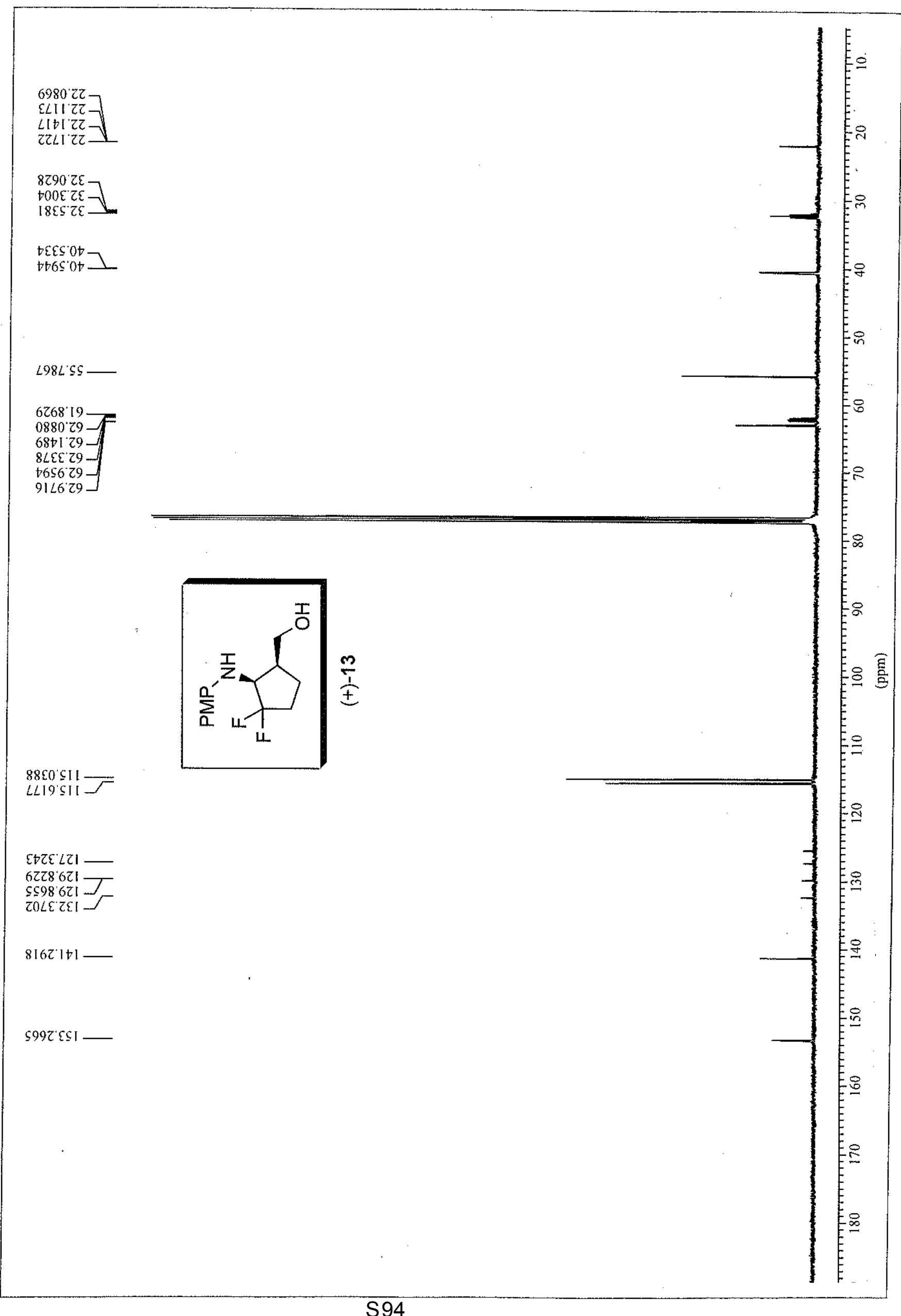




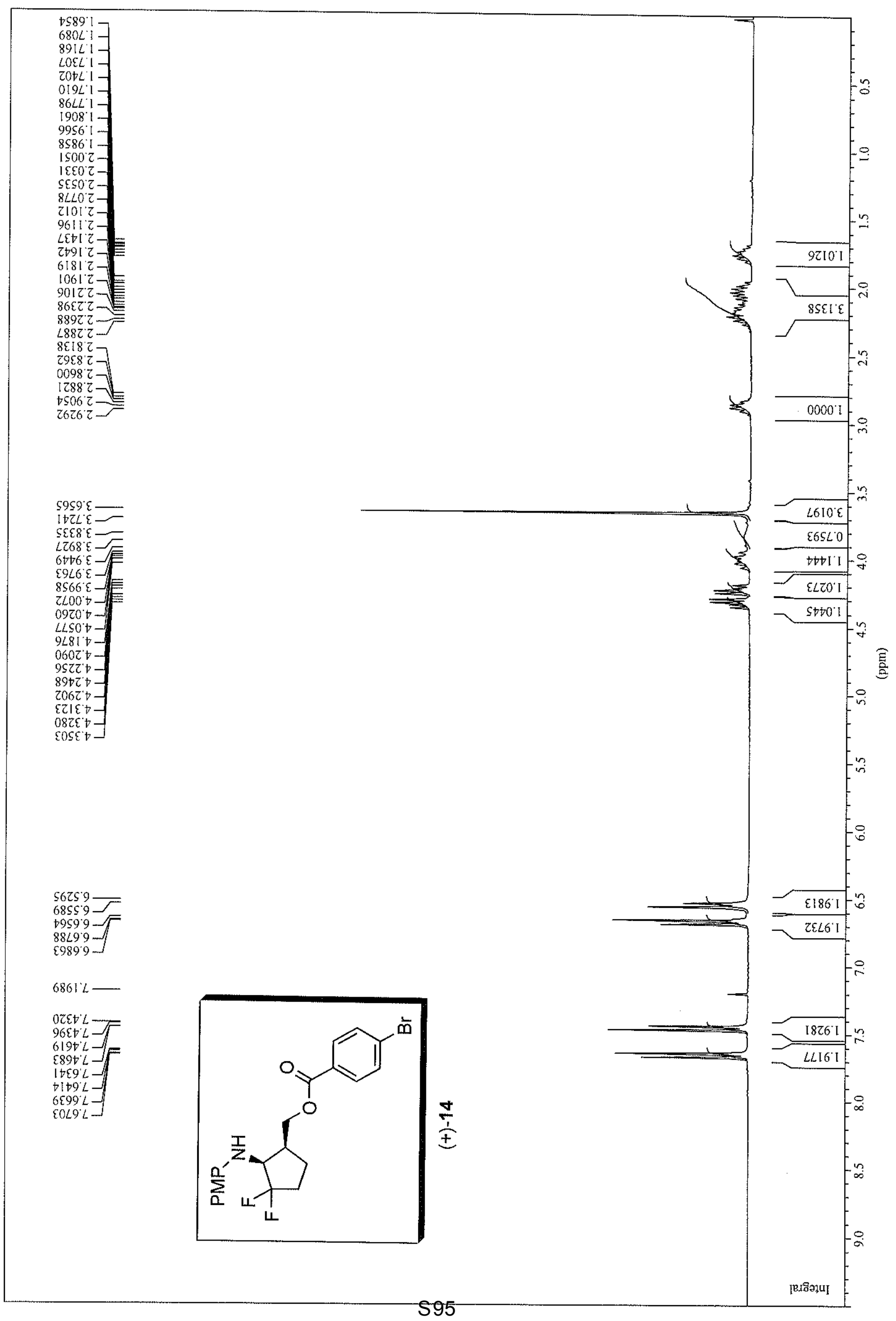


$v 296 \angle 01^{-}$

$8006 \mathrm{LOI}^{-}$

$61 \pm 8<01-$

66LL $\angle 01-$

$6151201-$
$2980^{\circ} \angle 01-$

$8<70<01-$

$\angle 590^{\circ} 901-$

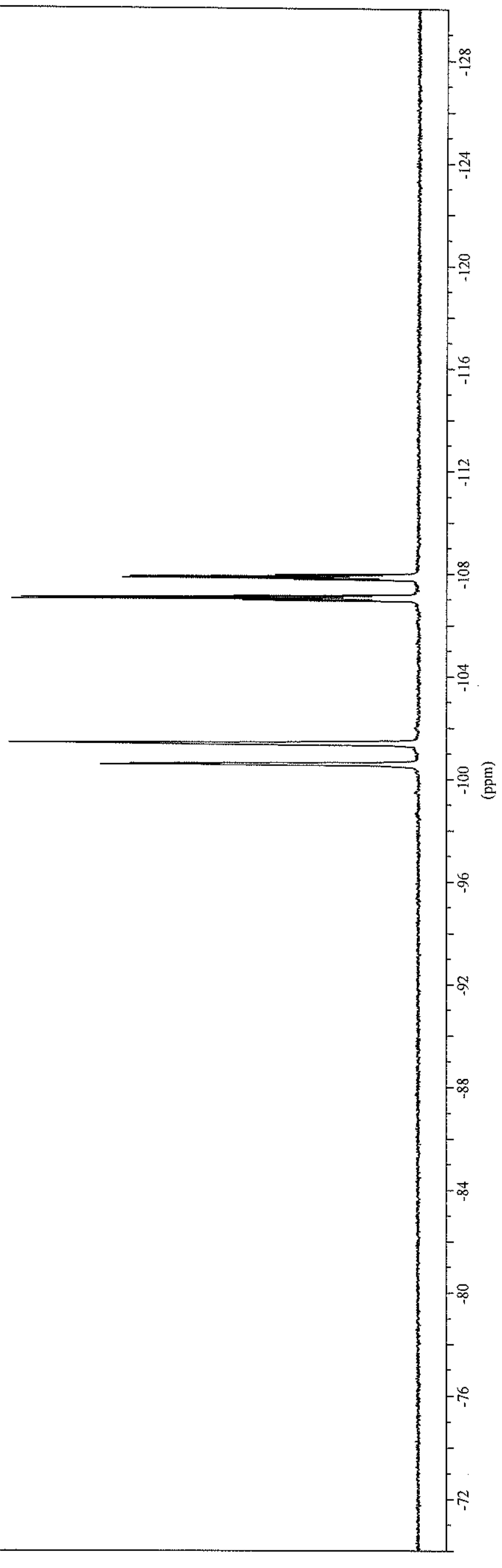

OESt $101^{-}$

S91tiol-

ZLDE:101".

$68 \varepsilon 9^{\circ} 001^{-}$

†209001-

$9695001^{-}$

OEES $001^{-}$

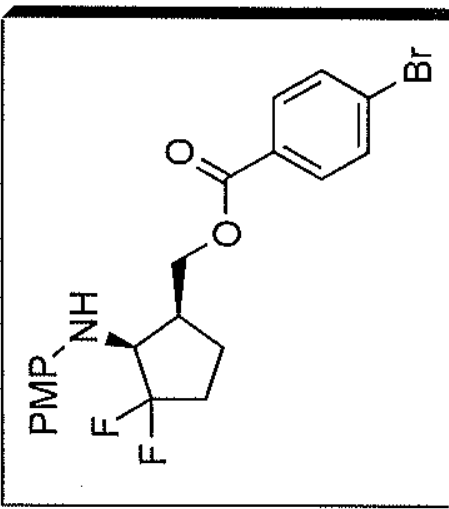

$\frac{⿱ 亠 䒑}{ \pm}$ 
$10 \varepsilon+2 z$

Itis IS

$1+\varepsilon 8 ! \varepsilon$

$69012 \varepsilon$

9lttit $2 \varepsilon$

68ZSLE $\longrightarrow$

L8E9'SS

$0 \angle S 8.65$

8811.09

916109

tESt.09

$18 z 2769$

LLLS $9 L$

$5666^{\circ} 9 \mathrm{~L}-\bar{P}$

I $802: L-$

987tiol

L28\&๋ाI

00L8'tII

$6 \$ 50.92 \mathrm{I}$

$\angle 8508 \mathrm{ZI}$

S189.821-

LGLE 621 -

IStti6z]

S100'I ]

6LGS [E] -

$689 L \tau \varepsilon 1$

$6189^{\circ} 001$ -

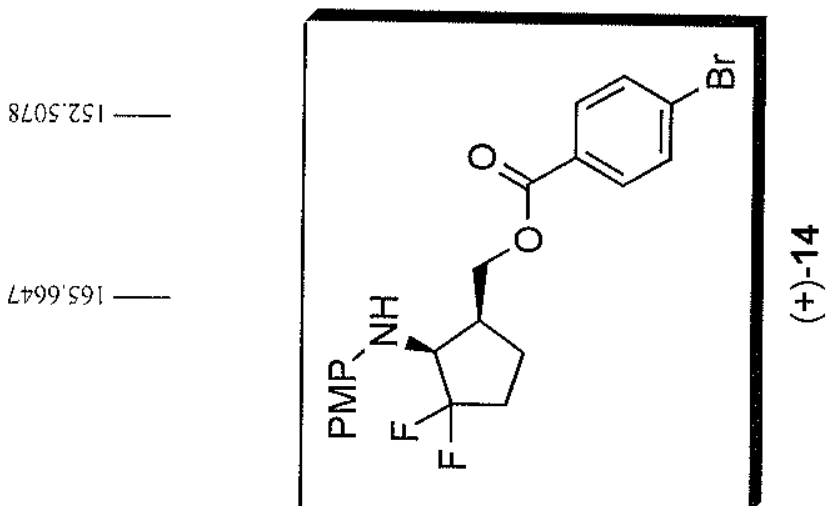

$\frac{⿱ 亠 䒑 十}{ \pm}$ 
$9181 !$

$2608^{\circ} 1-$

$6+\varepsilon 81$

c958 I

$0406 \div$

IELOZ

$\$ 660^{\circ} \mathrm{Z}$

9Izl 2

sovl'z.

$2891^{\prime} z$

66227

$\angle 88{ }^{\circ} z$

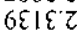

SoE⿷

$6 \triangleright 9 \varepsilon$

seoti

$7 \varepsilon 06^{\circ} \mathrm{z}$

SS26 2

$1606 \pi-\sqrt{5}$

SZL6 2

sotz.

\&S十 $\varepsilon \varepsilon-7$

ZOSTE-

$8687^{\circ} \mathrm{S}-$

$s \angle 00^{\circ}$

$0 \tau \nabla 0^{\circ} \nabla=\bar{F}$

EOLO'

zSOI't -

996 乙

t9\&ะ $v-$

$68 S \varepsilon \circ-$

$S S L E D$

$0910 t$

Gzist.

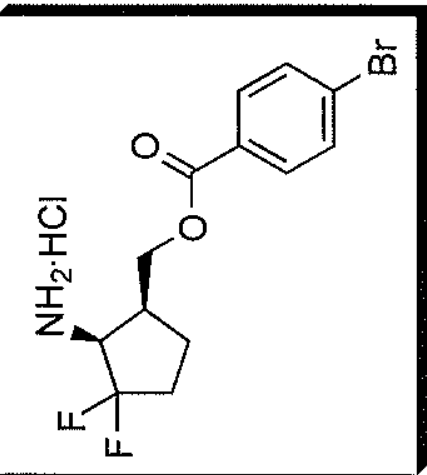

$\frac{10}{1}$

$1609^{\circ} \mathrm{L}-$

$\angle 9 E 9 L$

$09062=$

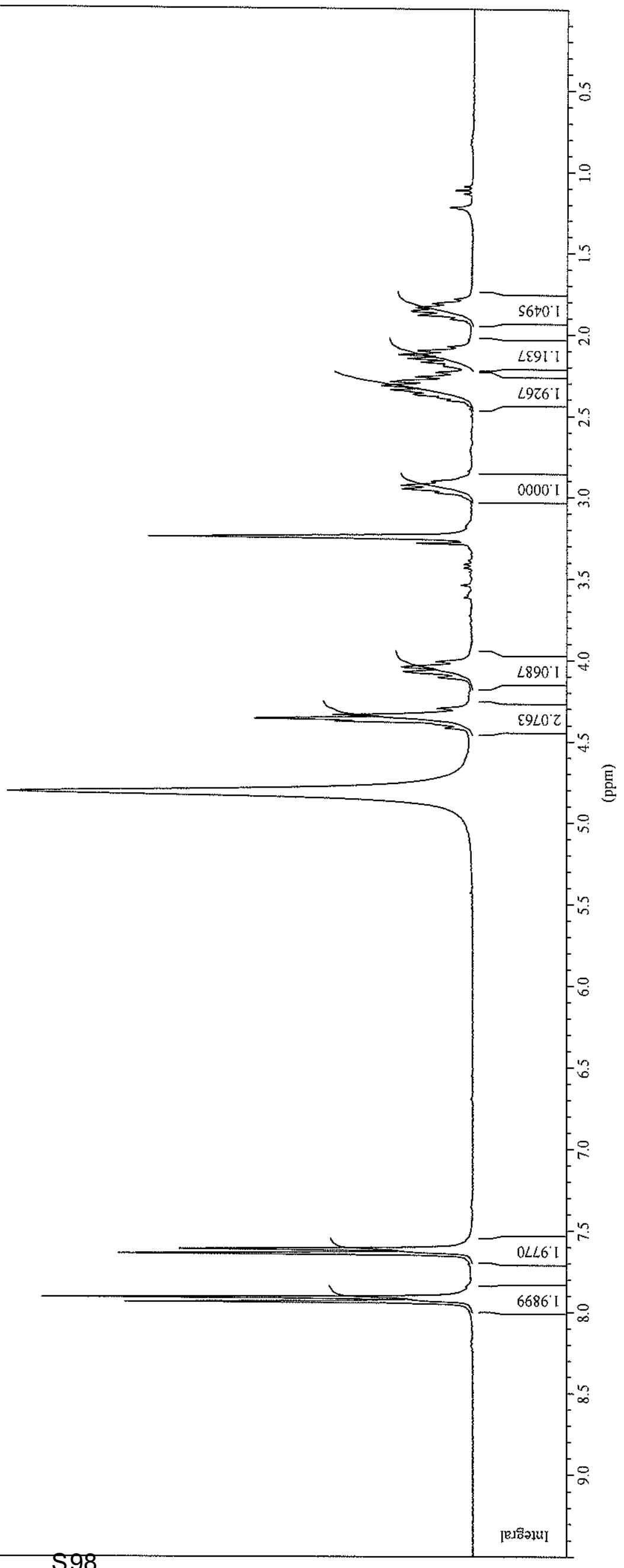


06てt $\varepsilon 01-$

ZE9E'E0I-

olgz'col-

E685 201-

9EZs $201-7$

$\forall \varepsilon 8 t^{2} \mathrm{zO} \mathrm{1}^{-}$

$\varepsilon|\tau \dagger z 0|-]$

$19+9^{\circ} \angle 6^{-}-$

$+908^{\circ} 96^{-}$

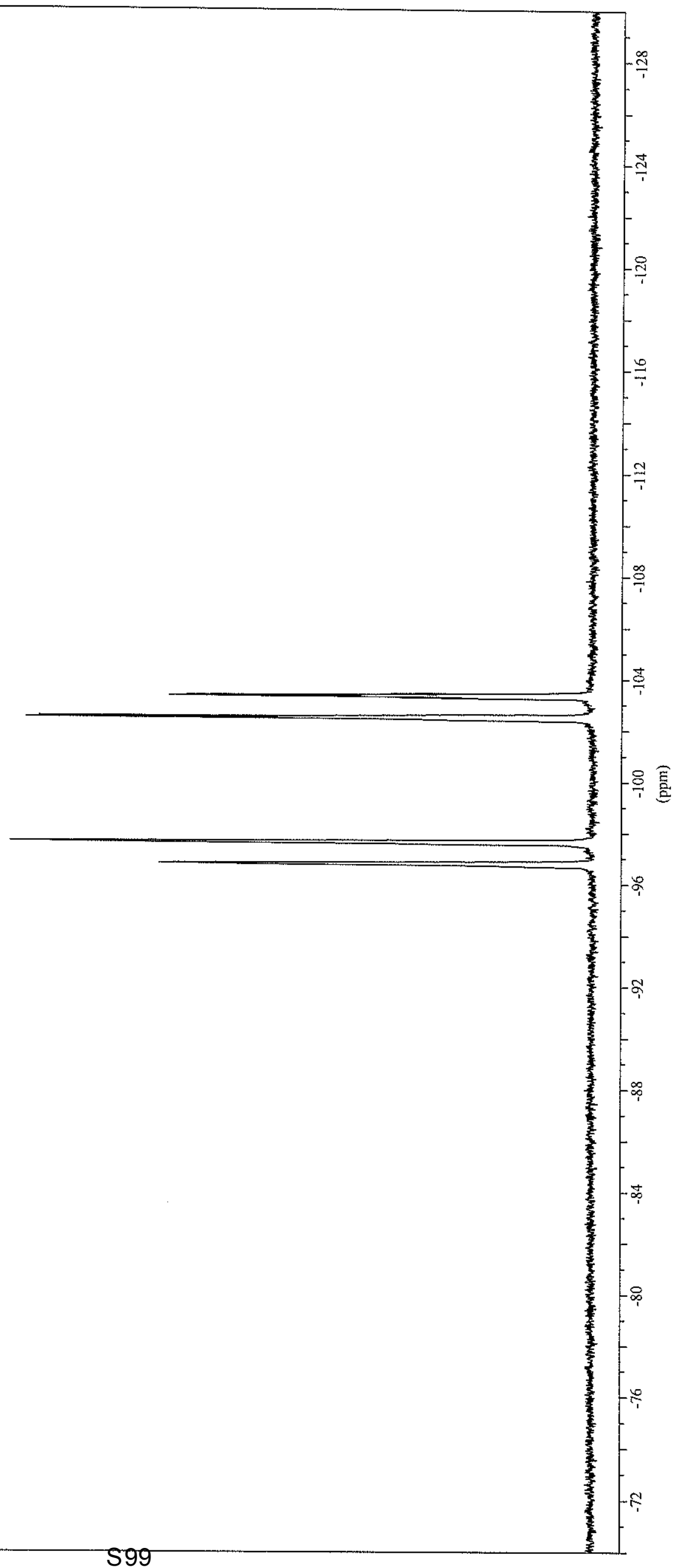


$66 S S^{\circ}+2$

SLZS $\mathcal{E}$

E0†8 $\varepsilon \varepsilon-$

OESI $\mathrm{QE}=$

$8 \angle 896 \varepsilon$

811060

$\rightarrow \subseteq 6 \tau^{\circ} 6 t-$

$16 L S 65$

L298 65

t9t1.0s

Oost os

$\angle E I L O S$ 를

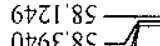

$0 \rightarrow 6585-7$

$0+62.85$

$2 \pm \varepsilon 0.59$

6t\&ELZI -

$\tau t+\tau 0 \varepsilon$ -

$\rightarrow$ toL OE $1=$

ISS8 OEI

GLZ££E -

$\dagger \varepsilon L 8 \varepsilon \varepsilon 1$

$\varsigma 290 \circ £ 1$

I $|S L L Q 9|=$
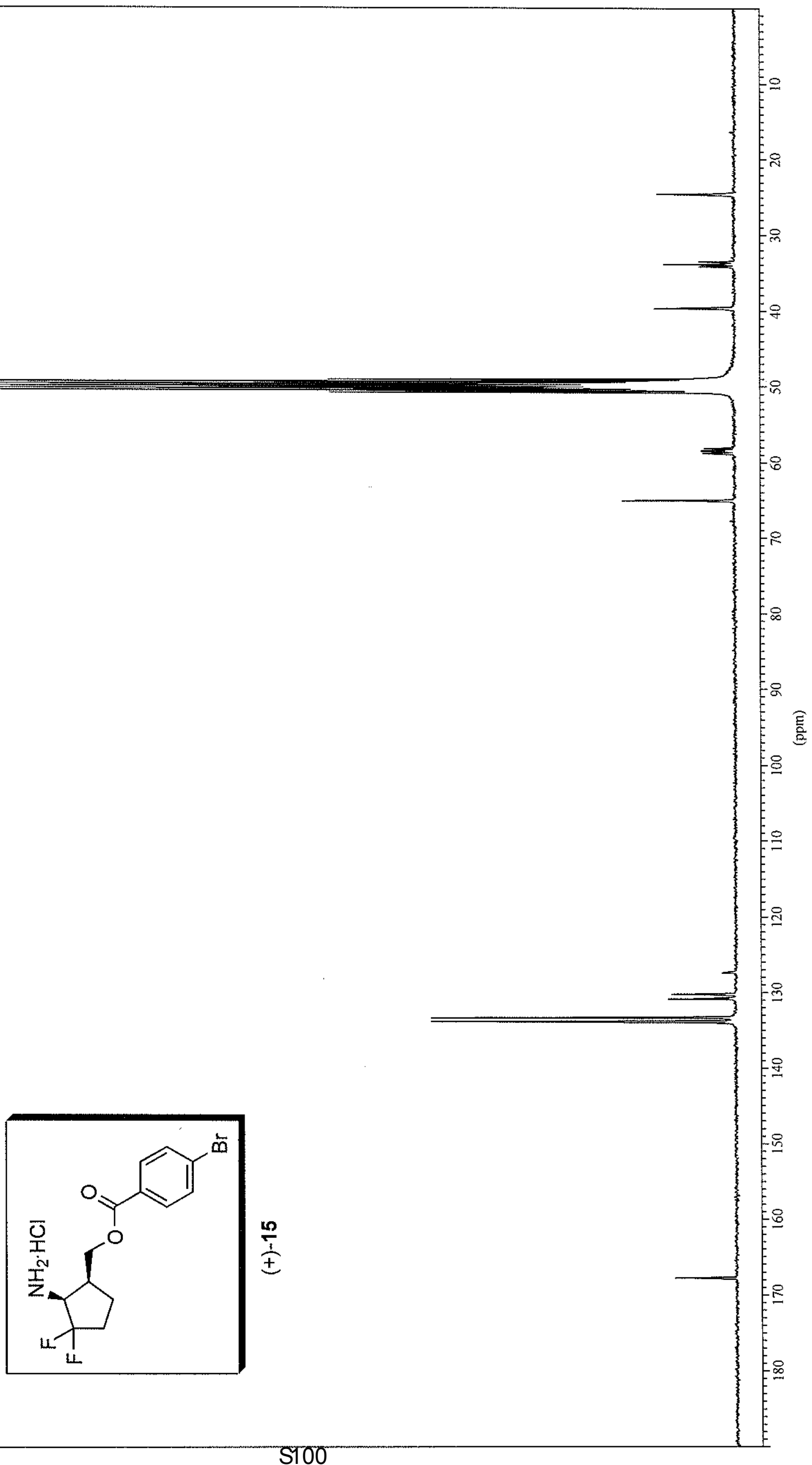